\title{
Global Smooth Supersonic Flows in Infinite Expanding Nozzles
}

\author{
Chunpeng Wang* \\ School of Mathematics, Jilin University, Changchun 130012, P. R. China \\ The Institute of Mathematical Sciences, The Chinese University of Hong Kong, Shatin, NT, Hong Kong \\ (email: wangcp@jlu.edu.cn) \\ Zhouping $\mathrm{Xin}^{\dagger}$ \\ The Institute of Mathematical Sciences and Department of Mathematics, \\ The Chinese University of Hong Kong, Shatin, NT, Hong Kong \\ (email: zpxin@ims.cuhk.edu.hk)
}

\begin{abstract}
This paper concerns smooth supersonic flows with Lipschitz continuous speed in two-dimensional infinite expanding nozzles, which are governed by a quasilinear hyperbolic equation being singular at the sonic and vacuum state. The flow satisfies the slip condition on the walls and the flow velocity is prescribed at the inlet. First, it is proved that if the incoming flow is away from the sonic and vacuum state and its streamlines are rarefactive at the inlet, then a flow in a straight nozzle never approaches the sonic and vacuum state in any bounded region. Furthermore, a sufficient and necessary condition of the incoming flow at the inlet is derived for the existence of a global smooth supersonic flow in a straight nozzle. Then, it is shown that for each incoming flow satisfying this condition, there exists uniquely a global smooth supersonic flow in a symmetric nozzle with convex upper wall. It is noted that such a flow may contain a vacuum. If there is a vacuum for a global smooth transonic flow in a symmetric nozzle with convex upper wall, it is proved that for the symmetric upper part of the flow, the first vacuum point along the symmetric axis must be located at the upper wall and the set of vacuum points is the closed domain bounded by the tangent halfline of the upper wall at this point to downstream and the upper wall after this point. Moreover, the flow speed is globally Lipschitz continuous in the nozzle, and on the boundary between the gas and the vacuum, the flow velocity is along this boundary and the normal derivatives of the flow speed and the square of the sound speed both are zero. As an immediate consequence, the local smooth transonic flow obtained in 10 can be extended into a global smooth transonic flow in a symmetric nozzle whose upper wall after the local flow is convex.
\end{abstract}

Keywords: Smooth supersonic flow, Singularity, Vacuum. 2000 MR Subject Classification: 76J20 76N10 35L20 35L81

\footnotetext{
${ }^{*}$ The research is supported by a grant from the National Natural Science Foundation of China (No. 11222106).

${ }^{\dagger}$ The research is supported by Zheng Ge Ru Foundation, Hong Kong RGC Earmarked Research Grants CUHK4041/11P and CUHK-4048/13P, a grant from the Croucher Foundation, and a Focus Area Grant from the Chinese University of Hong Kong.
} 


\section{Contents}

1 Introduction

2 Formulation of the smooth supersonic flow problem 6

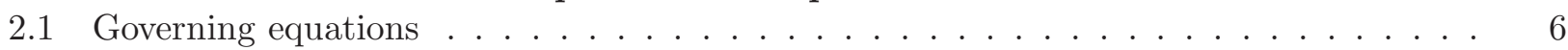

2.2 Smooth transonic flows in finite de Laval nozzles . . . . . . . . . . . . . . . 8

2.3 Formulation of the smooth supersonic flow problem . . . . . . . . . . . . . 11

2.4 Necessary condition at the inlet for a global smooth supersonic flow in a straight nozzle 14

2.5 On the global smooth transonic flow problem . . . . . . . . . . . . . 19

3 Smooth supersonic flows before vacuum formation 2

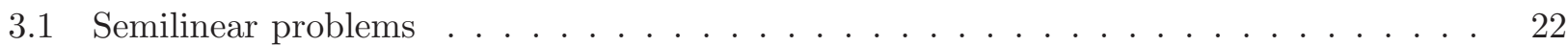

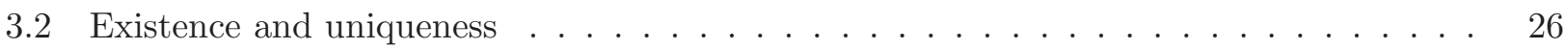

3.3 Global smooth supersonic flows without vacuum in straight nozzles . . . . . . . . . . . . 33

3.4 Properties of smooth supersonic flows before vacuum formation . . . . . . . . . . 35

4 Formation of vacuum in a smooth supersonic flow 39

4.1 Location of the first vacuum point and behavior of the flow near this point . . . . . 39

4.2 Sufficient conditions for formation of vacuum . . . . . . . . . . . . . 41

4.3 Lower bound estimates of the location of the first vacuum point . . . . . . . . . . 43

4.4 Formation of a shock in a nozzle whose upper wall is a non-convex perturbation of a straight line .................................. 45

5 Global smooth supersonic flows 46

5.1 Properties of the vacuum boundary . . . . . . . . . . . . . . . 47

5.2 Dynamics of the vacuum boundary and a free boundary problem . . . . . . . . . 48

5.3 Global smooth supersonic flows . . . . . . . . . . . . . . . . . 53

5.4 Smooth supersonic flows with vacuum at the inlet $\ldots \ldots \ldots \ldots$ 


\section{Introduction}

This paper concerns the global existence of smooth supersonic potential flows in two-dimensional infinite expanding nozzles, which arises naturally in physical experiments and engineering designs. Here, a smooth supersonic flow means that its velocity is continuous and its speed is Lipschitz continuous. Assume that there is a smooth incoming supersonic flow at the inlet of an infinite expanding nozzle. It is interesting to study whether the smooth supersonic flow exists globally or not. For such a global smooth supersonic flow, what is the asymptotic behavior of the flow at the downstream, is there a vacuum in the nozzle, what is the vacuum set and what is the behavior of the flow on the boundary between the gas and the vacuum? If a smooth supersonic flow does not exist globally, what is the singularity?

One of the main motivations of this paper lies in the global existence of smooth transonic flows in two-dimensional infinite de Laval nozzles. In [10], we studied smooth transonic potential flows of Meyer type in a class of two-dimensional finite de Laval nozzles. It was shown that a flow with nonexceptional points is unstable for a $C^{1}$ small perturbation in the shape of the nozzle and thus we sought smooth transonic flows of Meyer type whose sonic points are exceptional. It was proved that there exists uniquely such a smooth transonic flow near the throat of the nozzle, whose acceleration is Lipschitz continuous, if the wall of the nozzle is sufficiently flat. A natural question is whether this local smooth transonic flow can be extended smoothly and globally. For a supersonic flow, there is also a discontinuous extension pattern called a transonic shock, which is supersonic upstream and turn to subsonic across the transonic shock. Courant and Friedrichs had described such a pattern as follows ([5]). Given the appropriately large receiver pressure, if the upstream flow is still supersonic behind the throat of the nozzle, then at a certain place in the divergent part of the nozzle a shock front intervenes and the gas is compressed and slowed down to subsonic speed, which was proved rigidly in recent works [6, 7, 8, for two-dimensional full Euler system. See also [1, 2, 3, 4, 11] for related works. The reason of the intervention of a transonic shock in the pattern lies in that there is a large receiver pressure, which can be achieved only when the flow is subsonic, at the outlet of the nozzle. We are interested in the smooth extension of a supersonic flow in an infinite expanding nozzle when the flow is not required to be subsonic downstream. That is to say, it is concerned how to exclude transonic shocks, supersonic shocks and sonic state in the extension of a supersonic flow in an infinite expanding nozzle.

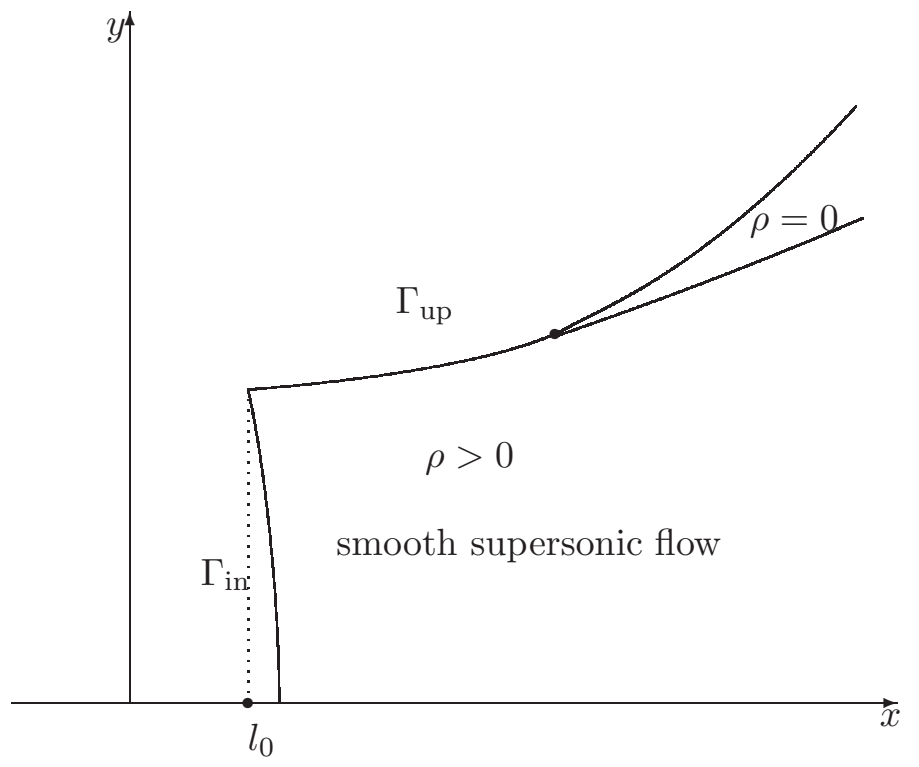


As usual, it is assumed that the nozzle is symmetric with respect to the $x$-axis. For convenience, we consider only the upper part of the nozzle. The upper wall and the inlet are given by $\Gamma_{\text {up }}: y=$ $f(x)\left(l_{0} \leq x<l_{1}\right)$ and $\Gamma_{\text {in }}: x=\Upsilon(y)\left(0 \leq y \leq f\left(l_{0}\right)\right)$, respectively, where $0<l_{0}<l_{1} \leq+\infty$, $f \in C^{2}\left(\left[l_{0}, l_{1}\right)\right), \Upsilon \in C^{2}\left(\left[0, f\left(l_{0}\right)\right]\right)$ and

$f\left(l_{0}\right)>0, \quad \lim _{x \rightarrow l_{1}^{-}}(x+f(x))=+\infty, \quad \inf _{\left(l_{0}, l_{1}\right)} f^{\prime} \geq 0, \quad \Upsilon\left(f\left(l_{0}\right)\right)=l_{0}, \quad \Upsilon^{\prime}(0)=0, \quad \Upsilon^{\prime}\left(f\left(l_{0}\right)\right)=-f^{\prime}\left(l_{0}\right)$.

Consider the smooth supersonic flow problem in the infinite nozzle $\Omega$ bounded by the upper wall $\Gamma_{\text {up }}$, the lower wall $x$-axis and the inlet $\Gamma_{\text {in }}$. The flow velocity is prescribed at the inlet. In particular, only the flow speed needs to be prescribed if it is assumed that the incoming flow velocity is along the normal direction at the inlet. The flow satisfies the slip condition on the walls. Therefore, the problem can be formulated as follows

$$
\begin{array}{ll}
\operatorname{div}\left(\rho\left(|\nabla \varphi|^{2}\right) \nabla \varphi\right)=0, & (x, y) \in \Omega, \\
\varphi(\Upsilon(y), y)=0, & 0<y<f\left(l_{0}\right), \\
|\nabla \varphi(\Upsilon(y), y)|=q_{0}(y), & 0<y<f\left(l_{0}\right), \\
\rho\left(|\nabla \varphi(x, 0)|^{2}\right) \frac{\partial \varphi}{\partial y}(x, 0)=0, & \Upsilon(0)<x<l_{1}, \\
\rho\left(|\nabla \varphi(x, f(x))|^{2}\right)\left(\frac{\partial \varphi}{\partial y}(x, f(x))-f^{\prime}(x) \frac{\partial \varphi}{\partial x}(x, f(x))\right)=0, & l_{0}<x<l_{1},
\end{array}
$$

where $\rho$ is a known function given by

$$
\rho\left(q^{2}\right)=\left(1-\frac{\gamma-1}{2} q^{2}\right)^{1 /(\gamma-1)}, \quad 0 \leq q \leq \sqrt{2 /(\gamma-1)} \quad(\gamma>1),
$$

$q_{0} \in C^{1}\left(\left[0, f\left(l_{0}\right)\right]\right)$ and it satisfies

$$
\sqrt{2 /(\gamma+1)}<\inf _{\left(0, f\left(l_{0}\right)\right)} q_{0} \leq \sup _{\left(0, f\left(l_{0}\right)\right)} q_{0}<\sqrt{2 /(\gamma-1)}
$$

which guarantees that the flow is away from the sonic and vacuum state at the inlet.

It is noted that (1.1) is a quasilinear hyperbolic equation in the supersonic region and is singular at sonic and vacuum points, where two characteristics coincide. For the special case that the nozzle is straight, it is shown that smooth supersonic flows to the problem (1.1)-(1.5) are away from vacuum in any bounded region. That is to say, for a supersonic flow in a straight nozzle, a vacuum never forms and thus the flow exists smoothly and globally if there is not a sonic point and a shock. It turns out that a natural choice to exclude the sonic state is that the streamlines of the incoming flow are rarefactive at the inlet, i.e.

$$
\Upsilon^{\prime \prime}(y) \leq 0, \quad 0<y<f\left(l_{0}\right)
$$

Indeed, the speed of a smooth supersonic flow in a straight nozzle is not less than the minimal speed of the incoming flow on the inlet if (1.6) is satisfied. Can (1.6) also exclude shocks for a supersonic flow in a straight nozzle? The answer turns to be no. If (1.6) is satisfied, a sufficient and necessary condition to exclude shocks for a supersonic flow in a straight nozzle is proved to be

$$
\left|q_{0}^{\prime}(y)\right| \leq \frac{-\Upsilon^{\prime \prime}(y)}{1+\left(\Upsilon^{\prime}(y)\right)^{2}} \sqrt{\frac{-q_{0}^{2}(y) \rho\left(q_{0}^{2}(y)\right)}{\rho\left(q_{0}^{2}(y)\right)+2 q_{0}^{2}(y) \rho^{\prime}\left(q_{0}^{2}(y)\right)}}, \quad 0<y<f\left(l_{0}\right),
$$


which also implies (1.6). Therefore, if $q_{0}$ satisfies (1.7), then the problem (1.1)-(1.5) in a straight nozzle admits uniquely a smooth supersonic flow which never approaches the sonic and vacuum state in any bounded region; further, the flow is shown to be accelerating along each streamline. If (1.7) is invalid, a sonic point or a shock must form in the flow; furthermore, if in addition, (1.6) holds, then the flow is away from the sonic state and its singularity is a shock. It is noted that the local smooth transonic flow obtained in [10] satisfies (1.7) at the outlet. So, this local flow can be extended into a global smooth transonic flow without vacuum if the upper wall of the nozzle after the outlet of the local flow is straight.

For a straight nozzle, (1.7) is a sufficient and necessary condition to get a global smooth supersonic flow. Thus, for a general expanding nozzle, we solve the problem (1.1)-(1.5) under the assumption (1.7). Generally speaking, the geometry of nozzles can create vacuum, sonic state and shocks. It is shown that for a nozzle whose upper wall is convex, the problem (1.1)-(1.5) admits uniquely a global smooth supersonic flow. The convexity of the upper wall of the nozzle is necessary in the following sense: if the upper wall of the nozzle is a nonconvex perturbation of a straight line, then there exists a $q_{0}$ satisfying (1.7) such that a shock must form for the supersonic flow in the nozzle. Such a global smooth supersonic flow in a nozzle with convex upper wall may contain a vacuum, and its acceleration is continuous in the gas region. If there is a vacuum, it is clear that the set of vacuum points is closed and thus there exists the first vacuum point in the increasing $x$-direction. It is shown that this first vacuum point must be located at the upper wall and the set of vacuum points is the closed domain bounded by the tangent half-line of the upper wall at this point to the downstream and the upper wall after this point. Moreover, this tangent half-line is a streamline of the flow. That is to say, the flow velocity at the boundary between the gas and the vacuum is along this boundary. For such a global smooth supersonic flow with vacuum, the flow speed is globally Lipschitz continuous in the nozzle and the normal derivatives of the flow speed and the square of the sound speed on the boundary between the gas and the vacuum both are zero. Therefore, the vacuum in the flow is not the so called physical vacuum in [9, 12. This is natural since there is not an external force on the flow.

The global existence result for smooth supersonic flows in a nozzle with convex upper wall shows that the geometry of the nozzle is essential for the formation of vacuum. For a nozzle whose upper wall is convex but not straight, if the incoming flow is near the vacuum, then a vacuum must form in the global smooth supersonic flow. Furthermore, the first vacuum point will tend to $\left(x_{*}, f\left(x_{*}\right)\right)$ with $x_{*}=\inf \left\{x \in\left[l_{0}, l_{1}\right): f^{\prime \prime}(x)>0\right\}$ as $q_{0}\left(f\left(l_{0}\right)\right)$ tends to $\sqrt{2 /(\gamma-1)}$. It is noted that there are a large class of nozzles in which a global smooth supersonic flow must contain a vacuum even if the incoming flow is near the sonic state. For example, if

$$
\begin{cases}\lim _{x \rightarrow+\infty} f^{\prime \prime}(x) x^{2 \gamma /(\gamma+1)}=+\infty, & \text { when } l_{1}=+\infty \text { and } f^{\prime}(+\infty)<+\infty, \\ \lim _{x \rightarrow+l_{1}} \frac{f^{\prime \prime}(x)}{\left(f^{\prime}(x)\right)^{3}} f^{2 \gamma /(\gamma+1)}(x)=+\infty, & \text { when } l_{1} \leq+\infty \text { and } f^{\prime}\left(l_{1}\right)=+\infty,\end{cases}
$$

then each global smooth supersonic flow to the problem (1.1)-(1.5) admits a vacuum provided that $q_{0}$ satisfies

$$
\left|q_{0}^{\prime}(y)\right|<\frac{-\Upsilon^{\prime \prime}(y)}{1+\left(\Upsilon^{\prime}(y)\right)^{2}} \sqrt{\frac{-q_{0}^{2}(y) \rho\left(q_{0}^{2}(y)\right)}{\rho\left(q_{0}^{2}(y)\right)+2 q_{0}^{2}(y) \rho^{\prime}\left(q_{0}^{2}(y)\right)}}, \quad 0 \leq y \leq f\left(l_{0}\right) .
$$

Before the appearance of a vacuum, the smooth supersonic flow problem is solved in the potential plane, where the domain is a rectangle and thus it is convenient to calculate the effect of the boundary condition. For a global smooth supersonic flow in a straight nozzle, a necessary condition of the incoming flow at the inlet is derived by a characteristic method. The method for the existence of 
smooth supersonic flows before vacuum formation is a fixed point argument. We use the method of characteristics to get a priori estimates for suitable semilinear problems and then use the fixed point theorem to get a solution to the quasilinear problem. However, the coordinates transformation between the physical coordinates and the potential-stream coordinates may be invalid in the appearance of vacuum. So, we solve the smooth supersonic flow problem with vacuum as a free boundary problem in the physical plane, where the boundary between the gas and the vacuum is free. It is proved that this free boundary is a streamline of the flow by studying the extension of the gas from the potential level set where the first vacuum forms.

The paper is arranged as follows. In $\S 2$ we formulate the global smooth supersonic flow problem in an infinite expanding nozzle with convex upper wall. Moreover, a necessary condition of the incoming flow at the inlet is derived for the existence of global smooth supersonic flows in a straight nozzle. In $\S 3$ we solve the maximal smooth supersonic flow problem before vacuum formation in a nozzle with convex upper wall. In particular, there exists uniquely a global smooth supersonic flow without vacuum if the nozzle is straight. Properties of smooth supersonic flows before vacuum formation are also studied. In $\S 4$ we investigate properties of the first vacuum point for a smooth supersonic flow and give some sufficient conditions for formation of a vacuum. Subsequently, we prove the global well-posedness for the smooth supersonic flow problem in an infinite nozzle with convex upper wall in $\S 5$.

\section{Formulation of the smooth supersonic flow problem}

In this section, we formulate the smooth supersonic flow problem in an infinite expanding nozzle. The focus is to characterize the geometry of the nozzles and the boundary conditions at their inlets to ensure the global existence of smooth supersonic flows in these nozzles. One of the main motivations is to extend the local smooth transonic flows in a de Laval nozzle obtained in [10] globally.

\subsection{Governing equations}

Steady isentropic compressible Euler flows satisfy

$$
\begin{aligned}
& \frac{\partial}{\partial x}(\rho u)+\frac{\partial}{\partial y}(\rho v)=0 \\
& \frac{\partial}{\partial x}\left(P+\rho u^{2}\right)+\frac{\partial}{\partial y}(\rho u v)=0 \\
& \frac{\partial}{\partial x}(\rho u v)+\frac{\partial}{\partial y}\left(P+\rho v^{2}\right)=0
\end{aligned}
$$

where $(u, v), P$ and $\rho$ represent the velocity, pressure and density of the flow, respectively. The flow is assumed to be isentropic so that $P=P(\rho)$ is a smooth function. In particular, for a polytropic gas with the adiabatic exponent $\gamma>1$,

$$
P(\rho)=\frac{1}{\gamma} \rho^{\gamma}
$$

is the normalized pressure. Assume further that the flow is irrotational, i.e.

$$
\frac{\partial u}{\partial y}=\frac{\partial v}{\partial x}
$$

Then the density $\rho$ is expressed in terms of the speed $q$ according to the Bernoulli law ([5])

$$
\rho\left(q^{2}\right)=\left(1-\frac{\gamma-1}{2} q^{2}\right)^{1 /(\gamma-1)}, \quad q=\sqrt{u^{2}+v^{2}}, \quad 0 \leq q \leq c^{*}=\sqrt{2 /(\gamma-1)} .
$$


The sound speed $c$ is defined as

$$
c^{2}=P^{\prime}(\rho)=\rho^{\gamma-1}=1-\frac{\gamma-1}{2} q^{2}, \quad 0 \leq q \leq c^{*} .
$$

At the sonic state, the sound speed is $c_{*}=\sqrt{2 /(\gamma+1)}$, which is called the sonic speed in the sense that the flow is subsonic $(q<c)$ when $0<q<c_{*}$, sonic $(q=c)$ when $q=c_{*}$ and supersonic $(q>c)$ when $q>c_{*}$.

Define a velocity potential $\varphi$ and a stream function $\psi$, respectively, by

$$
\frac{\partial \varphi}{\partial x}=u, \quad \frac{\partial \varphi}{\partial y}=v, \quad \frac{\partial \psi}{\partial x}=-\rho v, \quad \frac{\partial \psi}{\partial y}=\rho u
$$

which are

$$
\frac{\partial \varphi}{\partial x}=q \cos \theta, \quad \frac{\partial \varphi}{\partial y}=q \sin \theta, \quad \frac{\partial \psi}{\partial x}=-\rho q \sin \theta, \quad \frac{\partial \psi}{\partial y}=\rho q \cos \theta
$$

in terms of polar coordinators in the velocity space, where $\theta$, which is called a flow angle, is the angle of the velocity inclination to the $x$-axis. Direct calculations show that the system (2.1) $-(2.6)$ can be reduced to the full potential equation

$$
\operatorname{div}\left(\rho\left(|\nabla \varphi|^{2}\right) \nabla \varphi\right)=0
$$

in the physical coordinates $(x, y)$ and the Chaplygin equations

$$
\frac{\partial \theta}{\partial \psi}+\frac{\rho\left(q^{2}\right)+2 q^{2} \rho^{\prime}\left(q^{2}\right)}{q \rho^{2}\left(q^{2}\right)} \frac{\partial q}{\partial \varphi}=0, \quad \frac{1}{q} \frac{\partial q}{\partial \psi}-\frac{1}{\rho\left(q^{2}\right)} \frac{\partial \theta}{\partial \varphi}=0
$$

in the potential-stream coordinates $(\varphi, \psi)$. Note that

$$
\frac{\partial(\varphi, \psi)}{\partial(x, y)}=\frac{\partial \varphi}{\partial x} \frac{\partial \psi}{\partial y}-\frac{\partial \varphi}{\partial y} \frac{\partial \psi}{\partial x}=\rho q^{2} .
$$

So the coordinates transformation between the two coordinate systems is valid at least in the absence of stagnation and vacuum points. Eliminating $\theta$ from (2.8) yields the following second-order quasilinear equation

$$
\frac{\partial^{2} A(q)}{\partial \varphi^{2}}+\frac{\partial^{2} B(q)}{\partial \psi^{2}}=0
$$

with

$$
A(q)=\int_{c_{*}}^{q} \frac{\rho\left(s^{2}\right)+2 s^{2} \rho^{\prime}\left(s^{2}\right)}{s \rho^{2}\left(s^{2}\right)} d s, \quad B(q)=\int_{c_{*}}^{q} \frac{\rho\left(s^{2}\right)}{s} d s, \quad 0<q<c^{*} .
$$

Here, $B(\cdot)$ is strictly increasing in $\left(0, c^{*}\right)$, while $A(\cdot)$ is strictly increasing in $\left(0, c_{*}\right]$ and strictly decreasing in $\left[c_{*}, c^{*}\right)$. It can be checked easily that both (2.7) and (2.9) are elliptic in the subsonic region $\left(q<c_{*}\right)$ and hyperbolic in the supersonic region $\left(q>c_{*}\right)$, while singular at the sonic state $\left(q=c_{*}\right)$ and the vacuum $\left(q=c^{*}\right)$.

As shown in [10], in the supersonic region without vacuum, (2.9) can be rewritten as

$$
Q_{\varphi \varphi}-\left(b(Q) Q_{\psi}\right)_{\psi}=0
$$


or equivalently as

$$
\left\{\begin{array}{l}
W_{\varphi}+b^{1 / 2}(Q) W_{\psi}=\frac{1}{4} b^{-1}(Q) p(Q) W(W+Z) \\
Z_{\varphi}-b^{1 / 2}(Q) Z_{\psi}=-\frac{1}{4} b^{-1}(Q) p(Q) Z(W+Z)
\end{array}\right.
$$

where

$$
Q=A(q), \quad W=Q_{\varphi}-b^{1 / 2}(Q) Q_{\psi}, \quad Z=-Q_{\varphi}-b^{1 / 2}(Q) Q_{\psi}
$$

and

$$
\begin{gathered}
b(s)=\left.\left(\frac{\gamma+1}{2} q^{2}-1\right)^{-1}\left(1-\frac{\gamma-1}{2} q^{2}\right)^{2 /(\gamma-1)+1}\right|_{q=A_{+}^{-1}(s)}>0, \quad s<0, \\
p(s)=\left.(\gamma+1) q^{4}\left(\frac{\gamma+1}{2} q^{2}-1\right)^{-3}\left(1-\frac{\gamma-1}{2} q^{2}\right)^{3 /(\gamma-1)+1}\right|_{q=A_{+}^{-1}(s)}>0, \quad s<0
\end{gathered}
$$

with $A_{+}^{-1}$ being the inverse function of $A(\cdot)$ lying in $\left[c_{*}, c^{*}\right)$. It is noted that

$$
\lim _{s \rightarrow 0^{-}} s b^{-1}(s) p(s)=-\frac{1}{2}, \quad \lim _{s \rightarrow-\infty} s b^{-1}(s) p(s)=-(\gamma+1) .
$$

\subsection{Smooth transonic flows in finite de Laval nozzles}

Since one of the main motivations of this paper lies in the global existence of smooth transonic flows, we first recall the smooth transonic flow problem in finite de Laval nozzles investigated in [10] and show boundary conditions of such local smooth transonic flows at an outlet. This will yield the formulation of inlet boundary conditions for smooth supersonic flows in an expanding nozzle.

It was shown in [10] that a smooth transonic flow of Meyer type with nonexceptional points is unstable for a $C^{1}$ small perturbation on the wall (even if the wall is still smooth) and thus we sought ones whose sonic points are exceptional. For such a flow, its sonic curve must be located at the throat of the nozzle and where the potential becomes a constant identically. The smooth transonic flow problem in [10] was described physically as follows: for the upper wall $\Gamma_{\text {up }}: y=f(x)\left(l_{-} \leq x \leq l_{+}\right)$ and for the inlet $\Gamma_{\text {in }}: x=g(y)\left(0 \leq y \leq f\left(l_{-}\right), g\left(f\left(l_{-}\right)\right)=l_{-}\right)$, seek a smooth transonic flow of Meyer type in the nozzle $\Omega$ bounded by the upper wall $\Gamma_{\text {up }}$, the lower wall $x$-axis, the inlet $\Gamma_{\text {in }}$ and the outlet $\Gamma_{\text {out }}: x=\Upsilon(y)\left(0 \leq y \leq f\left(l_{+}\right), \Upsilon\left(f\left(l_{+}\right)\right)=l_{+}\right)$which is a free boundary, such that its velocity is along the normal direction at the inlet and the outlet, it satisfies the slip condition on the walls and its sonic curve is located at the throat where the potential is equal to zero identically. Mathematically, the problem was formulated as

$$
\begin{array}{ll}
\operatorname{div}\left(\rho\left(|\nabla \varphi|^{2}\right) \nabla \varphi\right)=0, & (x, y) \in \Omega, \\
\varphi(g(y), y)=C_{\text {in }}, & 0<y<f\left(l_{-}\right), \\
\frac{\partial \varphi}{\partial y}(x, 0)=0, & g(0)<x<\Upsilon(0), \\
\frac{\partial \varphi}{\partial y}(x, f(x))-f^{\prime}(x) \frac{\partial \varphi}{\partial x}(x, f(x))=0, & l_{-}<x<l_{+}, \\
\varphi(\Upsilon(y), y)=C_{\text {out }}, & 0<y<f\left(l_{+}\right), \\
\varphi(0, y)=0,|\nabla \varphi(0, y)|=c_{*}, & 0<y<f(0), \\
|\nabla \varphi(x, y)|<c_{*}, & (x, y) \in \Omega \text { and } x<0, \\
|\nabla \varphi(x, y)|>c_{*}, & (x, y) \in \Omega \text { and } x>0,
\end{array}
$$


where $C_{\mathrm{in}}$ and $C_{\text {out }}$ are free constants, and $\Gamma_{\text {out }}$ is a free boundary.

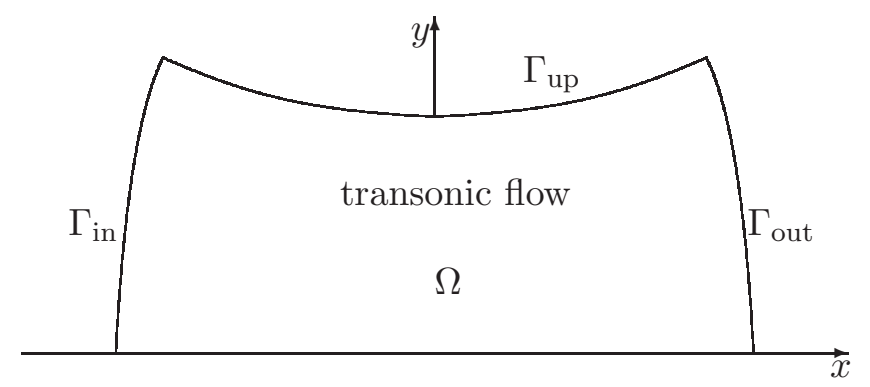

The smooth transonic flow problem was solved locally in the potential plane in [10]. It follows from (2.16) that the mass flux of the flow is $m=f(0) c_{*}^{1+2 /(\gamma-1)}$. Denote the flow speed at the inlet and at the upper wall by

$$
q(g(y), y)=\mathscr{Q}_{\text {in }}(y), \quad 0 \leq y \leq f\left(l_{-}\right) \quad \text { and } \quad q(x, f(x))=\mathscr{Q}_{\mathrm{up}}(x), \quad l_{-} \leq x \leq l_{+},
$$

respectively. The stream function at the inlet and the potential function at the upper wall can be given by

$$
\psi(g(y), y)=\Psi_{\text {in }}(y)=\int_{0}^{y} \mathscr{Q}_{\text {in }}(s) \rho\left(\mathscr{Q}_{\text {in }}^{2}(s)\right) \sqrt{1+\left(g^{\prime}(s)\right)^{2}} d s, \quad 0 \leq y \leq f\left(l_{-}\right)
$$

and

$$
\varphi(x, f(x))=\Phi_{\mathrm{up}}(x)=\int_{0}^{x} \mathscr{Q}_{\mathrm{up}}(s) \sqrt{1+\left(f^{\prime}(s)\right)^{2}} d s, \quad l_{-} \leq x \leq l_{+},
$$

respectively. Then, in the potential plane, the problem (2.11)-(2.18) is changed to

$$
\begin{array}{ll}
\frac{\partial^{2} A(q)}{\partial \varphi^{2}}+\frac{\partial^{2} B(q)}{\partial \psi^{2}}=0, & (\varphi, \psi) \in\left(\zeta_{-}, \zeta_{+}\right) \times(0, m), \\
\frac{\partial A(q)}{\partial \varphi}\left(\zeta_{-}, \psi\right)=\left.\frac{g^{\prime \prime}(y)\left(1+\left(g^{\prime}(y)\right)^{2}\right)^{-3 / 2}}{\mathscr{Q}_{\mathrm{in}}(y) \rho\left(\mathscr{Q}_{\mathrm{in}}^{2}(y)\right)}\right|_{y=Y_{\mathrm{in}}(\psi)}, & \psi \in(0, m), \\
\frac{\partial q}{\partial \psi}(\varphi, 0)=0, & \varphi \in\left(\zeta_{-}, \zeta_{+}\right), \\
\frac{\partial B(q)}{\partial \psi}(\varphi, m)=\left.\frac{f^{\prime \prime}(x)\left(1+\left(f^{\prime}(x)\right)^{2}\right)^{-3 / 2}}{\mathscr{Q}_{\mathrm{up}}(x)}\right|_{x=X_{\mathrm{up}}(\varphi)}, & \varphi \in\left(\zeta_{-}, \zeta_{+}\right), \\
q(0, \psi)=c_{*}, & \psi \in(0, m), \\
0<q(\varphi, \psi)<c_{*}, & (\varphi, \psi) \in\left(\zeta_{-}, 0\right) \times(0, m), \\
c_{*}<q(\varphi, \psi)<c^{*}, & (\varphi, \psi) \in\left(0, \zeta_{+}\right) \times(0, m), \\
\mathscr{Q}_{\mathrm{in}}(y)=\left.q(0, \psi)\right|_{\psi=\Psi_{\text {in }}(y)}, & y \in\left(0, f_{-}\left(l_{-}\right)\right), \\
\mathscr{Q}_{\mathrm{up}}(x)=\left.q(\varphi, m)\right|_{\varphi=\Phi_{\mathrm{up}}(x)}, & x \in\left(l_{-}, l_{+}\right),
\end{array}
$$

where $\zeta_{ \pm}=\Phi_{\text {up }}\left(l_{ \pm}\right), Y_{\text {in }}$ and $X_{\text {up }}$ are the inverse functions of $\Psi_{\text {in }}$ and $\Phi_{\text {up }}$, respectively.

The main results in [10] are the following existence and uniqueness. 
Theorem 2.1 [10] Assume that $f \in C^{4}\left(\left[l_{-}, l_{+}\right]\right)\left(-1 \leq l_{-}<0<l_{+} \leq 1\right)$ satisfies $f(0)>0$,

$$
\begin{gathered}
\delta_{1,-}(-x)^{\lambda_{-}} \leq f^{\prime \prime}(x) \leq \delta_{2,-}(-x)^{\lambda_{-}}, \quad\left|f^{(3)}(x)\right| \leq \delta_{3,-}(-x)^{\lambda_{-} / 4+1 / 2}, \quad\left|f^{(4)}(x)\right| \leq \delta_{4,-}, \quad l_{-} \leq x \leq 0, \\
\delta_{1,+} x^{\lambda_{+}} \leq f^{\prime \prime}(x) \leq \delta_{2,+} x^{\lambda_{+}}, \quad\left|f^{(3)}(x)\right| \leq \delta_{3,+} x^{\lambda_{+}-1}, \quad 0 \leq x \leq l_{+},
\end{gathered}
$$

and $g \in C^{3, \alpha}\left(\left[0, f\left(l_{-}\right)\right]\right)(0<\alpha<1)$ satisfies

$$
\begin{gathered}
g^{\prime}(0)=0, \quad g\left(f\left(l_{-}\right)\right)=l_{-}, \quad g^{\prime}\left(f\left(l_{-}\right)\right)=-f^{\prime}\left(l_{-}\right), \quad \sup _{\left(0, f\left(l_{-}\right)\right)}\left|\left(\frac{g^{\prime}}{\sqrt{1+\left(g^{\prime}\right)^{2}}}\right)^{\prime \prime}\right| \leq \epsilon\left(l_{-}\right)\left(-l_{-}\right)^{3 \lambda_{-} / 2}, \\
\frac{1}{2} \leq \frac{f\left(l_{-}\right) \sqrt{\left(f^{\prime}\left(l_{-}\right)\right)^{2}+1}}{-f^{\prime}\left(l_{-}\right)}\left(\frac{g^{\prime}(y)}{\sqrt{1+\left(g^{\prime}(y)\right)^{2}}}\right)^{\prime} \leq \frac{3}{2}, \quad 0 \leq y \leq f\left(l_{-}\right),
\end{gathered}
$$

where $\lambda_{ \pm}, \delta_{i, \pm}(i=1,2,3)$ and $\delta_{4,-}$ are positive constants such that $\lambda_{ \pm}>2$ and $\delta_{1, \pm} \leq \delta_{2, \pm}$, while $0<\epsilon(s) \leq 1$ for $-1 \leq s<0$ and $\lim _{s \rightarrow 0^{-}} \epsilon(s)=0$. Then, there exist two positive constants $\delta_{0,-}$ (depending only on $\gamma, m, \lambda_{-}, \delta_{1,-}, \delta_{2,-}$ and $\epsilon(\cdot)$ ) and $\delta_{0,+}$ (depending only on $\gamma, m, \lambda_{+}, \delta_{1,+}, \delta_{2,+}$ and $\delta_{3,+}$ ) such that if $-\delta_{0,-} \leq l_{-}<0<l_{+} \leq \delta_{0,+}$, then the problem (2.19)-(2.27) admits a unique solution $q \in C^{1,1}\left(\left[\zeta_{-}, \zeta_{+}\right] \times[0, m]\right)$ satisfying

(i) On $\left[\zeta_{-}, 0\right] \times[0, m], q$ satisfies

$$
\begin{gathered}
c_{*}-C_{2,-}(-\varphi)^{\lambda_{-} / 2+1} \leq q(\varphi, \psi) \leq c_{*}-C_{1,-}(-\varphi)^{\lambda_{-} / 2+1}, \\
\left|\frac{\partial q}{\partial \varphi}(\varphi, \psi)\right| \leq C_{3,-}(-\varphi)^{\lambda_{-} / 4+1 / 2}, \quad\left|\frac{\partial q}{\partial \psi}(\varphi, \psi)\right| \leq C_{3,-}(-\varphi)^{\lambda_{-} / 2+1}, \\
\left|\frac{\partial^{2} q}{\partial \varphi^{2}}(\varphi, \psi)\right| \leq C_{4,-}, \quad\left|\frac{\partial^{2} q}{\partial \varphi \partial \psi}(\varphi, \psi)\right| \leq C_{4,-}(-\varphi)^{\lambda_{-} / 4+1 / 2}, \quad\left|\frac{\partial^{2} q}{\partial \psi^{2}}(\varphi, \psi)\right| \leq C_{4,-}(-\varphi)^{\lambda_{-} / 2+1},
\end{gathered}
$$

where $C_{i,-}(i=1,2,3,4)\left(C_{1,-} \leq C_{2,-}\right)$ are positive constants depending only on $\gamma, m, \lambda_{-}, \delta_{1,-}$ and $\delta_{2,-}$, and $C_{3,-}$ also on $\delta_{3,-}$, while $C_{4,-}$ also on $\delta_{3,-}, \delta_{4,-}$ and $\alpha$.

(ii) $O n\left[0, \zeta_{+}\right] \times[0, m], Q=A(q)$ satisfies

$$
\begin{gathered}
-C_{2,+} \varphi^{\lambda_{+}+2} \leq Q(\varphi, \psi) \leq-C_{1,+} \varphi^{\lambda_{+}+2}, \\
-C_{2,+} \varphi^{\lambda_{+}+1} \leq Q_{\varphi}(\varphi, \psi) \leq-C_{1,+} \varphi^{\lambda_{+}+1}, \quad\left|Q_{\psi}(\varphi, \psi)\right| \leq C_{2,+} \varphi^{3 \lambda_{+} / 2+1}, \\
\left|Q_{\varphi \varphi}(\varphi, \psi)\right| \leq C_{3,+} \varphi^{\lambda_{+}}, \quad\left|Q_{\varphi \psi}(\varphi, \psi)\right| \leq C_{3,+} \varphi^{5 \lambda_{+} / 4+1 / 2}, \quad\left|Q_{\psi \psi}(\varphi, \psi)\right| \leq C_{3,+} \varphi^{3 \lambda_{+} / 2+1},
\end{gathered}
$$

where $C_{i,+}(i=1,2,3)\left(C_{1,+} \leq C_{2,+}\right)$ are positive constants depending only on $\gamma, m, \lambda_{+}, \delta_{1,+}$ and $\delta_{2,+}$, while $C_{3,+}$ also on $\delta_{3,+}$.

Theorem 2.2 [10] Under the assumptions of Theorem [2.1, the transonic flow problem (2.11) -(2.18) admits a unique smooth solution $\varphi \in C^{2,1}(\bar{\Omega})$, which satisfies Theorem 2.1 (i) for $q=|\nabla \varphi|$ in $\{(x, y) \in$ $\Omega: x<0\}$, while satisfies Theorem [2.1 (ii) for $Q=A(|\nabla \varphi|)$ in $\{(x, y) \in \Omega: x>0\}$.

We now characterize the outlet $\Gamma_{\text {out }}: x=\Upsilon(y)\left(0 \leq y \leq f\left(l_{+}\right)\right)$, which is a free boundary, and the conditions for the transonic flow at it. If $l_{+}$is small, Theorem 2.1 shows that at the outlet of the de Laval nozzle, the smooth transonic flow satisfies $Q\left(\zeta_{+}, \cdot\right) \in C^{1,1}([0, m])$ and

$$
Q\left(\zeta_{+}, \psi\right)<0, \quad-Q_{\varphi}\left(\zeta_{+}, \psi\right)-b^{1 / 2}\left(Q\left(\zeta_{+}, \psi\right)\right)\left|Q_{\psi}\left(\zeta_{+}, \psi\right)\right|>0, \quad 0 \leq \psi \leq m .
$$

Therefore, the outlet satisfies $\Upsilon \in C^{2,1}\left(\left[0, f\left(l_{+}\right)\right]\right)$and

$$
\Upsilon\left(f\left(l_{+}\right)\right)=l_{+}, \quad \Upsilon^{\prime}(0)=0, \quad \Upsilon^{\prime}\left(f\left(l_{+}\right)\right)=-f^{\prime}\left(l_{+}\right), \quad \sup _{\left(0, f\left(l_{+}\right)\right)} \Upsilon^{\prime \prime}<0
$$


and the flow speed at the outlet in the potential plane satisfies $q_{0} \in C^{1,1}\left(\left[0, f\left(l_{+}\right)\right]\right)$and

$$
c_{*}<q_{0}(y)<c^{*}, \quad\left|q_{0}^{\prime}(y)\right|<\frac{-\Upsilon^{\prime \prime}(y)}{1+\left(\Upsilon^{\prime}(y)\right)^{2}} \sqrt{\frac{-q_{0}^{2}(y) \rho\left(q_{0}^{2}(y)\right)}{\rho\left(q_{0}^{2}(y)\right)+2 q_{0}^{2}(y) \rho^{\prime}\left(q_{0}^{2}(y)\right)}}, \quad 0 \leq y \leq f\left(l_{+}\right)
$$

with

$$
q_{0}(y)=|\nabla \varphi(\Upsilon(y), y)|, \quad y \in\left[0, f\left(l_{+}\right)\right]
$$

\subsection{Formulation of the smooth supersonic flow problem}

Assume that $f \in C^{2}\left(\left[l_{0}, l_{1}\right)\right)\left(0<l_{0}<l_{1} \leq+\infty\right)$ satisfies

$$
f\left(l_{0}\right)>0, \quad \lim _{x \rightarrow l_{1}^{-}}(x+f(x))=+\infty, \quad f^{\prime}\left(l_{0}\right) \geq 0, \quad \inf _{\left(l_{0}, l_{1}\right)} f^{\prime \prime} \geq 0 .
$$

Give an inlet $\Gamma_{\text {in }}: x=\Upsilon(y)\left(0 \leq y \leq f\left(l_{0}\right), \Upsilon\left(f\left(l_{0}\right)\right)=l_{0}\right)$. We study the smooth supersonic flow problem in the unbounded nozzle $\Omega$ bounded by the upper wall $\Gamma_{\text {up }}: y=f(x)\left(l_{0} \leq x<l_{1}\right)$, the lower wall $x$-axis and the inlet $\Gamma_{\text {in }}: x=\Upsilon(y)\left(0 \leq y \leq f\left(l_{0}\right)\right)$. At the inlet, the flow velocity is prescribed and it is assumed to be along the normal direction of the inlet for convenience. On the walls, the flow

satisfies the slip condition. Here, a smooth supersonic flow means that its velocity is continuous and its speed is Lipschitz continuous.

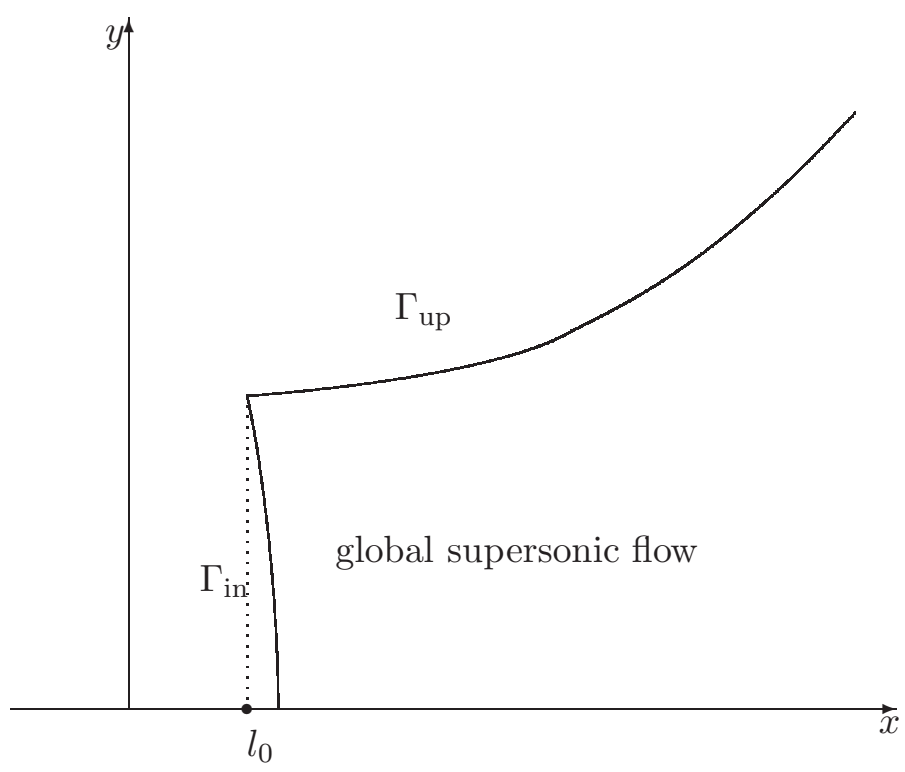

The smooth supersonic flow problem can be formulated as follows

$$
\begin{array}{ll}
\operatorname{div}\left(\rho\left(|\nabla \varphi|^{2}\right) \nabla \varphi\right)=0, & (x, y) \in \Omega, \\
\varphi(\Upsilon(y), y)=0, & 0<y<f\left(l_{0}\right), \\
|\nabla \varphi(\Upsilon(y), y)|=q_{0}(y), & 0<y<f\left(l_{0}\right) \\
\rho\left(|\nabla \varphi(x, 0)|^{2}\right) \frac{\partial \varphi}{\partial y}(x, 0)=0, & \Upsilon(0)<x<l^{2} \\
\rho\left(|\nabla \varphi(x, f(x))|^{2}\right)\left(\frac{\partial \varphi}{\partial y}(x, f(x))-f^{\prime}(x) \frac{\partial \varphi}{\partial x}(x, f(x))\right)=0, & l_{0}<x<l_{1}
\end{array}
$$


where $q_{0}$ is a given function defined on $\left[0, f\left(l_{0}\right)\right]$. Motivated by conditions (2.28) and (2.29) for local smooth transonic flows obtained in Theorem 2.2. we will solve the problem (2.31)-(2.35) under the assumptions that $\Upsilon \in C^{2}\left(\left[0, f\left(l_{0}\right)\right]\right)$ satisfies

$$
\Upsilon\left(f\left(l_{0}\right)\right)=l_{0}, \quad \Upsilon^{\prime}(0)=0, \quad \Upsilon^{\prime}\left(f\left(l_{0}\right)\right)=-f^{\prime}\left(l_{0}\right), \quad \sup _{\left(0, f\left(l_{0}\right)\right)} \Upsilon^{\prime \prime} \leq 0
$$

and $q_{0} \in C^{1}\left(\left[0, f\left(l_{0}\right)\right]\right)$ satisfies

$$
\begin{gathered}
c_{1} \leq q_{0}(y) \leq c_{2}, \quad 0<y<f\left(l_{0}\right), \\
\left|q_{0}^{\prime}(y)\right| \leq \frac{-\Upsilon^{\prime \prime}(y)}{1+\left(\Upsilon^{\prime}(y)\right)^{2}} \sqrt{\frac{-q_{0}^{2}(y) \rho\left(q_{0}^{2}(y)\right)}{\rho\left(q_{0}^{2}(y)\right)+2 q_{0}^{2}(y) \rho^{\prime}\left(q_{0}^{2}(y)\right)}}, \quad 0<y<f\left(l_{0}\right)
\end{gathered}
$$

and the compatible condition

$$
q_{0}^{\prime}(0)=0, \quad q_{0}^{\prime}\left(f\left(l_{0}\right)\right)=\frac{f^{\prime \prime}\left(l_{0}\right)}{1+\left(f^{\prime}\left(l_{0}\right)\right)^{2}} q_{0}\left(f\left(l_{0}\right)\right),
$$

where $c_{*}<c_{1} \leq c_{2}<c^{*}$ are two constants.

Definition 2.1 A function $\varphi$ is called to be a solution to the problem (2.31) -(2.35), if $\varphi \in C^{1}(\bar{\Omega})$ with $|\nabla \varphi| \in C^{0,1}(\bar{\Omega})$ and

$$
c_{*}<|\nabla \varphi(x, y)| \leq c^{*}, \quad(x, y) \in \Omega,
$$

and $\varphi$ satisfies (2.31) in the distribution sense and satisfies (2.32) $-(2.35)$ pointwisely.

Remark 2.1 Since the density of a supersonic flow is zero in the vacuum region, the uniqueness of the solution to the problem (2.31) -(2.35) means that the density and momentum are the same.

Remark 2.2 It is proved in Theorem 5.2 that for the smooth supersonic flow to the problem (2.31)(2.35), its speed belongs to $C^{1}(\Omega)$; furthermore, its momentum also belongs to $C^{1}(\Omega)$ in the case $1<\gamma \leq 2$.

Remark 2.3 For a supersonic flow to the problem (2.31)-(2.35), (2.31) is hyperbolic and the $x$-axis is regarded as the time axis. So, one can also consider the supersonic flow problem in a finite nozzle.

Remark 2.4 The inequality in (2.36) means that streamlines are rarefactive at the inlet, which is a natural choice to exclude the sonic state in the flow, as seen in Lemma 2.3.

Remark 2.5 As we will show in Remark 2.10 and Theorem 4.6, conditions (2.38) and (2.30) are optimal in the following sense: (2.38) is sufficient and necessary for the global existence of smooth supersonic flows in a straight nozzle; while if the upper wall of the nozzle is a nonconvex perturbation of a straight line, then there is a $q_{0} \in C^{1}\left(\left[0, f\left(l_{0}\right)\right]\right)$ satisfying (2.37) -(2.39) such that a shock must form for the supersonic flow to the problem (2.31)-(2.35).

If there is no vacuum, the smooth supersonic flow problem (2.31)-(2.35) can be treated more conveniently in the potential plane. It is assumed that $(\Upsilon(0), 0)$ is transformed into $(0,0)$ in the coordinates transformation from the physical plane to the potential plane. The mass flux and the stream function at the inlet can be given by

$$
m=\int_{0}^{f\left(l_{0}\right)} q_{0}(y) \rho\left(q_{0}^{2}(y)\right) \sqrt{1+\left(\Upsilon^{\prime}(y)\right)^{2}} d y
$$


and

$$
\psi(\Upsilon(y), y)=\Psi_{\text {in }}(y)=\int_{0}^{y} q_{0}(s) \rho\left(q_{0}^{2}(s)\right) \sqrt{1+\left(\Upsilon^{\prime}(s)\right)^{2}} d s, \quad 0 \leq y \leq f\left(l_{0}\right),
$$

respectively. If the flow speed at the upper wall is

$$
q(x, f(x))=\mathscr{Q}_{\mathrm{up}}(x), \quad l_{0} \leq x<l_{1},
$$

then the potential function at the upper wall is

$$
\varphi(x, f(x))=\Phi_{\mathrm{up}}(x)=\int_{l_{0}}^{x} \mathscr{Q}_{\mathrm{up}}(s) \sqrt{1+\left(f^{\prime}(s)\right)^{2}} d s, \quad l_{0} \leq x<l_{1} .
$$

Therefore, in the potential plane, the smooth supersonic flow problem (2.31)-(2.35) can be formulated as follows

$$
\begin{array}{ll}
Q_{\varphi \varphi}-\left(b(Q) Q_{\psi}\right)_{\psi}=0, & (\varphi, \psi) \in(0, \zeta) \times(0, m), \\
Q(0, \psi)=Q_{0}(\psi), & \psi \in(0, m), \\
Q_{\varphi}(0, \psi)=G_{0}(\psi), & \psi \in(0, m), \\
Q_{\psi}(\varphi, 0)=0, & \varphi \in(0, \zeta), \\
Q_{\psi}(\varphi, m)=-\frac{f^{\prime \prime}\left(X_{\mathrm{up}}(\varphi)\right)\left(1+\left(f^{\prime}\left(X_{\mathrm{up}}(\varphi)\right)\right)^{2}\right)^{-3 / 2}}{b(Q(\varphi, m))) A_{+}^{-1}(Q(\varphi, m))} & \varphi \in(0, \zeta), \\
\mathscr{Q}_{\mathrm{up}}(x)=A_{+}^{-1}\left(Q\left(\Phi_{\mathrm{up}}(x), m\right)\right), & x \in\left(l_{0}, l\right),
\end{array}
$$

where $\zeta=\Phi_{\text {up }}(l)\left(l_{0}<l \leq l_{1}\right), X_{\text {up }}$ is the inverse function of $\Phi_{\text {up }}$ and a solution $Q$ is a negative function. Moreover,

$$
Q_{0}(\psi)=\left.A\left(q_{0}(y)\right)\right|_{y=Y_{\text {in }}(\psi)}, \quad G_{0}(\psi)=\left.\frac{\Upsilon^{\prime \prime}(y)\left(1+\left(\Upsilon^{\prime}(y)\right)^{2}\right)^{-3 / 2}}{q_{0}(y) \rho\left(q_{0}^{2}(y)\right)}\right|_{y=Y_{\text {in }}(\psi)}, \quad 0<\psi<m
$$

with $Y_{\text {in }}$ being the inverse function of $\Psi_{\text {in. }}$ It follows from (2.37)-(2.39) and (2.46) that $Q_{0} \in C^{1}([0, m])$ and $G_{0} \in C([0, m])$ satisfy

$$
-\mu_{2} \leq Q_{0}(\psi) \leq-\mu_{1}, \quad 0 \leq-G_{0}(\psi) \pm b^{1 / 2}\left(Q_{0}(\psi)\right) Q_{0}^{\prime}(\psi) \leq \mu_{3}, \quad \psi \in(0, m)
$$

with three constants $0<\mu_{1} \leq \mu_{2}$ and $\mu_{3}>0$, and

$$
Q_{0}^{\prime}(0)=0, \quad Q_{0}^{\prime}(m)=-\frac{f^{\prime \prime}\left(l_{0}\right)\left(1+\left(f^{\prime}\left(l_{0}\right)^{2}\right)^{-3 / 2}\right.}{b\left(Q_{0}(m)\right) A_{+}^{-1}\left(Q_{0}(m)\right)} .
$$

It can be verified easily that the problem $(2.40)-(2.45)$ is equivalent to

$$
\begin{array}{ll}
W_{\varphi}+b^{1 / 2}(Q) W_{\psi}=\frac{1}{4} b^{-1}(Q) p(Q) W(W+Z), & (\varphi, \psi) \in(0, \zeta) \times(0, m), \\
Z_{\varphi}-b^{1 / 2}(Q) Z_{\psi}=-\frac{1}{4} b^{-1}(Q) p(Q) Z(W+Z), & (\varphi, \psi) \in(0, \zeta) \times(0, m), \\
W(0, \psi)=W_{0}(\psi)=G_{0}(\psi)-b^{1 / 2}\left(Q_{0}(\psi)\right) Q_{0}^{\prime}(\psi), & \psi \in(0, m), \\
Z(0, \psi)=Z_{0}(\psi)=-G_{0}(\psi)-b^{1 / 2}\left(Q_{0}(\psi)\right) Q_{0}^{\prime}(\psi), & \psi \in(0, m), \\
W(\varphi, 0)+Z(\varphi, 0)=0, & \varphi \in(0, \zeta),
\end{array}
$$




$$
\begin{array}{ll}
W(\varphi, m)+Z(\varphi, m)=\frac{2 f^{\prime \prime}\left(X_{\mathrm{up}}(\varphi)\right)\left(1+\left(f^{\prime}\left(X_{\mathrm{up}}(\varphi)\right)\right)^{2}\right)^{-3 / 2}}{b^{1 / 2}(Q(\varphi, m)) A_{+}^{-1}(Q(\varphi, m))}, & \varphi \in(0, \zeta), \\
Q_{\varphi}=\frac{1}{2}(W-Z), & (\varphi, \psi) \in(0, \zeta) \times(0, m), \\
Q(0, \psi)=Q_{0}(\psi), & \psi \in(0, m), \\
\mathscr{Q}_{\text {up }}(x)=A_{+}^{-1}\left(Q\left(\Phi_{\text {up }}(x), m\right)\right), & x \in\left(l_{0}, l\right) .
\end{array}
$$

It follows from (2.47) that $Q_{0} \in C^{1}([0, m])$ and $W_{0}, Z_{0} \in C([0, m])$ satisfy

$$
\begin{gathered}
-\mu_{2} \leq Q_{0}(\psi) \leq-\mu_{1}, \quad \psi \in(0, m) \\
-\mu_{3} \leq W_{0}(\psi) \leq 0, \quad 0 \leq Z_{0}(\psi) \leq \mu_{3}, \quad \psi \in(0, m) .
\end{gathered}
$$

\subsection{Necessary condition at the inlet for a global smooth supersonic flow in a straight nozzle}

We first study the formation of the singularity of supersonic flows to the Cauchy problem

$$
\begin{array}{ll}
Q_{\varphi \varphi}-\left(b(Q) Q_{\psi}\right)_{\psi}=0, & (\varphi, \psi) \in(0,+\infty) \times \mathbb{R}, \\
Q(0, \psi)=Q_{0}(\psi), & \psi \in \mathbb{R}, \\
Q_{\varphi}(0, \psi)=G_{0}(\psi), & \psi \in \mathbb{R} .
\end{array}
$$

Recall the following lemma for invariant quantities on characteristics.

Lemma 2.1 ([10]) Let $\Sigma_{+}\left(\Sigma_{-}\right)$be a positive (negative) characteristic for a $C^{1}$ supersonic flow without vacuum. Then, $\mathscr{H}(Q) \mp \theta$ is invariant on $\Sigma_{ \pm}$, where

$$
\mathscr{H}(s)=\int_{s}^{0} b^{1 / 2}(s) d t, \quad s \leq 0
$$

Remark 2.6 Note that $\mathscr{H}(-\infty)=\lim _{s \rightarrow-\infty} \mathscr{H}(s)$ exists and is bounded.

The following three lemmas will be needed.

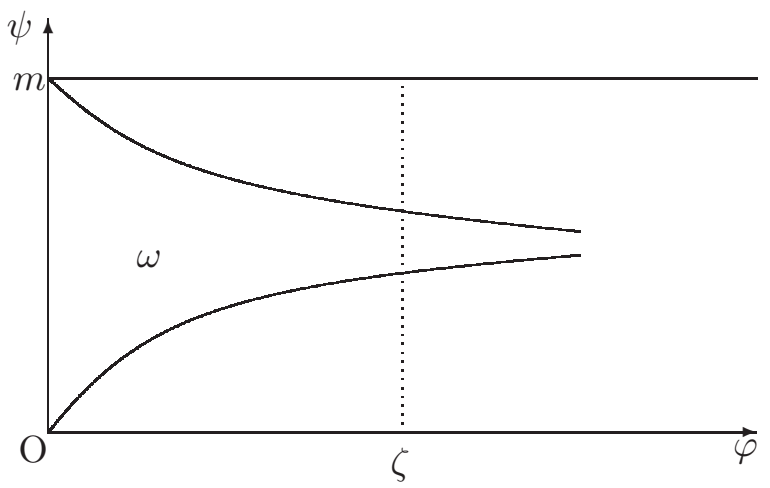

Lemma 2.2 For given $\zeta>0, Q_{0} \in C^{1}([0, m])$ with $\sup Q_{0}<0$ and $G_{0} \in C([0, m])$, assume that $Q \in C^{1}(\bar{\omega})$ solves

$$
Q_{\varphi \varphi}-\left(b(Q) Q_{\psi}\right)_{\psi}=0, \quad(\varphi, \psi) \in \omega
$$




$$
\begin{array}{ll}
Q(\zeta, \psi)=Q_{0}(\psi), & \psi \in(0, m), \\
Q_{\varphi}(\zeta, \psi)=G_{0}(\psi), & \psi \in(0, m),
\end{array}
$$

where $\omega$ is the domain bounded by the line $\varphi=0$, the line $\varphi=\zeta$, the positive characteristic from $(0,0)$ and the negative characteristic from $(0, m)$. Then

$$
-Q_{\varphi}(\varphi, \psi) \pm b^{1 / 2}(Q(\varphi, \psi)) Q_{\psi}(\varphi, \psi) \leq \max \left\{0, \sup _{(0, m)}\left(-G_{0}+b^{1 / 2}\left(Q_{0}\right)\left|Q_{0}^{\prime}\right|\right)\right\}, \quad(\varphi, \psi) \in \omega .
$$

If $\sup _{(0, m)} G_{0} \leq 0$ additionally, then $\sup _{\omega} Q=\sup _{(0, m)} Q_{0}$.

Proof. For $(\varphi, \psi) \in \bar{\omega}$, set

$W(\varphi, \psi)=Q_{\varphi}(\varphi, \psi)-b^{1 / 2}(Q(\varphi, \psi)) Q_{\psi}(\varphi, \psi), \quad Z(\varphi, \psi)=-Q_{\varphi}(\varphi, \psi)-b^{1 / 2}(Q(\varphi, \psi)) Q_{\psi}(\varphi, \psi)$.

Then, $(W, Z) \in C(\bar{\omega}) \times C(\bar{\omega})$ solving

$$
\begin{array}{ll}
W_{\varphi}+b^{1 / 2}(Q) W_{\psi}=\frac{1}{4} b^{-1}(Q) p(Q) W(W+Z), & (\varphi, \psi) \in \omega, \\
Z_{\varphi}-b^{1 / 2}(Q) Z_{\psi}=-\frac{1}{4} b^{-1}(Q) p(Q) Z(W+Z), & (\varphi, \psi) \in \omega, \\
W(0, \psi)=W_{0}(\psi)=G_{0}(\psi)-b^{1 / 2}\left(Q_{0}(\psi)\right) Q_{0}^{\prime}(\psi), & \psi \in(0, m), \\
Z(0, \psi)=Z_{0}(\psi)=-G_{0}(\psi)-b^{1 / 2}\left(Q_{0}(\psi)\right) Q_{0}^{\prime}(\psi), & \psi \in(0, m) .
\end{array}
$$

Denote

$$
M=\max \left\{0,-\inf _{(0, m)} W_{0}, \sup _{(0, m)} Z_{0}\right\}=\max \left\{0, \sup _{(0, m)}\left(-G_{0}+b^{1 / 2}\left(Q_{0}\right)\left|Q_{0}^{\prime}\right|\right)\right\}
$$

It suffices to prove that

$$
W(\varphi, \psi) \geq-M, \quad Z(\varphi, \psi) \leq M, \quad(\varphi, \psi) \in \omega
$$

If (2.64) is invalid, then one of the following two cases occurs:

(i) There exist two constants $\varphi_{+}>0$ and $M_{+}>M$, as well as a positive characteristic

$$
\Sigma_{+}: \Psi_{+}^{\prime}(\varphi)=b^{1 / 2}\left(Q\left(\varphi, \Psi_{+}(\varphi)\right)\right), \quad 0<\varphi<\varphi_{+}
$$

such that $W\left(\varphi_{+}, \Psi_{+}\left(\varphi_{+}\right)\right)=-M_{+}$and $Z\left(\cdot, \Psi_{+}(\cdot)\right) \leq M_{+}$on $\left[0, \varphi_{+}\right]$.

(ii) There exist two constants $\varphi_{-}>0$ and $M_{-}>M$, as well as a negative characteristic

$$
\Sigma_{-}: \Psi_{-}^{\prime}(\varphi)=-b^{1 / 2}\left(Q\left(\varphi, \Psi_{-}(\varphi)\right)\right), \quad 0<\varphi<\varphi_{-}
$$

such that $Z\left(\varphi_{-}, \Psi_{-}\left(\varphi_{-}\right)\right)=M_{-}$and $W\left(\cdot, \Psi_{-}(\cdot)\right) \geq-M_{-}$on $\left[0, \varphi_{-}\right]$.

Without loss of generality, it is assumed that (i) occurs. On $\Sigma_{+}, W$ satisfies

$$
\frac{d}{d \varphi} W\left(\varphi, \Psi_{+}(\varphi)\right)=R(\varphi) W\left(\varphi, \Psi_{+}(\varphi)\right)\left(W\left(\varphi, \Psi_{+}(\varphi)\right)+Z\left(\varphi, \Psi_{+}(\varphi)\right)\right), \quad 0<\varphi<\varphi_{+},
$$

where

$$
R(\varphi)=\frac{1}{4} b^{-1}\left(Q\left(\varphi, \Psi_{+}(\varphi)\right)\right) p\left(Q\left(\varphi, \Psi_{+}(\varphi)\right)\right), \quad 0 \leq \varphi \leq \varphi_{+}
$$


Since $W\left(\varphi_{+}, \Psi_{+}\left(\varphi_{+}\right)\right)=-M_{+}<0,(2.65)$ shows $W\left(\cdot, \Psi_{+}(\cdot)\right)<0$ on $\left[0, \varphi_{+}\right]$. Rewrite (2.65) as

$$
\begin{aligned}
& \left(W\left(\varphi, \Psi_{+}(\varphi)\right) \exp \left\{-\int_{0}^{\varphi} R(s) W\left(s, \Psi_{+}(s)\right) d s\right\}\right)^{\prime} \\
= & -Z\left(\varphi, \Psi_{+}(\varphi)\right)\left(\exp \left\{-\int_{0}^{\varphi} R(s) W\left(s, \Psi_{+}(s)\right) d s\right\}\right)^{\prime}, \quad 0<\varphi<\varphi_{+},
\end{aligned}
$$

which, together with $Z\left(\cdot, \Psi_{+}(\cdot)\right) \leq M_{+}$and $W\left(\cdot, \Psi_{+}(\cdot)\right)<0$ on $\left[0, \varphi_{+}\right]$, leads to

$$
\begin{aligned}
& \left(W\left(\varphi, \Psi_{+}(\varphi)\right) \exp \left\{-\int_{0}^{\varphi} R(s) W\left(s, \Psi_{+}(s)\right) d s\right\}\right)^{\prime} \\
\geq & -M_{+}\left(\exp \left\{-\int_{0}^{\varphi} R(s) W\left(s, \Psi_{+}(s)\right) d s\right\}\right)^{\prime}, \quad 0<\varphi<\varphi_{+} .
\end{aligned}
$$

Therefore,

$$
W_{0}\left(\Psi_{+}(0)\right)+M_{+} \leq\left(W\left(\varphi_{+}, \Psi_{+}\left(\varphi_{+}\right)\right)+M_{+}\right) \exp \left\{-\int_{0}^{\varphi_{+}} R(s) W\left(s, \Psi_{+}(s)\right) d s\right\}=0,
$$

which contradicts (2.63) and $M_{+}>M$. Hence (2.64) holds.

Below it is assumed that $\sup G_{0} \leq 0$ additionally, which implies $\theta_{\psi}(0, \cdot) \geq 0$ in $(0, m)$. Fix $(\tilde{\varphi}, \tilde{\psi}) \in \omega$. Assume that

$$
\tilde{\Sigma}_{ \pm}: \tilde{\Psi}_{ \pm}^{\prime}(\varphi)= \pm b^{1 / 2}\left(Q\left(\varphi, \tilde{\Psi}_{ \pm}(\varphi)\right)\right), \quad 0<\varphi<\tilde{\varphi}, \quad \tilde{\Psi}_{ \pm}(\tilde{\varphi})=\tilde{\psi}
$$

are the positive and negative characteristics approaching $(\tilde{\varphi}, \tilde{\psi})$. It follows from Lemma 2.1 that

$$
\mathscr{H}\left(Q_{0}\left(\tilde{\Psi}_{ \pm}(0)\right)\right) \mp \theta\left(0, \tilde{\Psi}_{ \pm}(0)\right)=\mathscr{H}(Q(\tilde{\varphi}, \tilde{\psi})) \mp \theta(\tilde{\varphi}, \tilde{\psi}) .
$$

Therefore,

$$
2 \mathscr{H}(Q(\tilde{\varphi}, \tilde{\psi}))=\mathscr{H}\left(Q_{0}\left(\tilde{\Psi}_{+}(0)\right)\right)+\mathscr{H}\left(Q_{0}\left(\tilde{\Psi}_{-}(0)\right)\right)-\theta\left(0, \tilde{\Psi}_{+}(0)\right)+\theta\left(0, \tilde{\Psi}_{-}(0)\right),
$$

which, together with $\theta_{\psi}(0, \cdot) \geq 0$ in $(0, m)$, leads to

$$
2 \mathscr{H}(Q(\tilde{\varphi}, \tilde{\psi})) \geq \mathscr{H}\left(Q_{0}\left(\Psi_{+}(0)\right)\right)+\mathscr{H}\left(Q_{0}\left(\Psi_{-}(0)\right)\right) \geq 2 \mathscr{H}\left(\sup _{(0, m)} Q_{0}\right) .
$$

Then, $\sup _{\omega} Q=\sup _{(0, m)} Q_{0}$ follows from the arbitrariness of $(\tilde{\varphi}, \tilde{\psi}) \in \omega$.

Lemma 2.3 Assume that $Q \in C^{1}([0, \zeta] \times[0, m])$ is a solution to the problem (2.40)-(2.45). If $\inf _{\left(l_{0}, l_{1}\right)} f^{\prime}=f^{\prime}\left(l_{0}\right)$ and $\sup _{\left(0, f\left(l_{0}\right)\right)} \Upsilon^{\prime \prime} \leq 0$, then $\inf _{(0, \zeta) \times(0, m)} A_{+}^{-1}(Q)=\inf _{\left(0, f\left(l_{0}\right)\right)} q_{0}$.

Proof. Note that

$$
\theta(\varphi, 0)=0=\inf _{(0, m)} \theta(0, \cdot), \quad \theta(\varphi, m) \geq \theta(0, m)=\sup _{(0, m)} \theta(0, \cdot), \quad 0 \leq \varphi \leq m .
$$

It follows from the method of characteristics and Lemma 2.1 that

$$
Q(\varphi, 0) \leq \sup _{(0, m)} Q_{0}, \quad Q(\varphi, m) \leq \sup _{(0, m)} Q_{0}, \quad 0 \leq \varphi \leq m .
$$

Then, a similar argument as the end of the proof of Lemma 2.2 leads $\sup _{(0, \zeta) \times(0, m)} Q=\sup _{(0, m)} Q_{0}$. 
Lemma 2.4 Assume that $Q \in C^{1}((0,+\infty) \times \mathbb{R})$ solves (2.60) and satisfies

$$
-M(\varphi+1)^{2} \leq Q(\varphi, \psi)<0, \quad(\varphi, \psi) \in(0,+\infty) \times \mathbb{R}
$$

with $M>0$ being a constant. Then,

$$
-Q_{\varphi}(\varphi, \psi) \pm b^{1 / 2}(Q(\varphi, \psi)) Q_{\psi}(\varphi, \psi) \geq 0, \quad(\varphi, \psi) \in(0,+\infty) \times \mathbb{R} .
$$

Proof. For $(\varphi, \psi) \in(0,+\infty) \times \mathbb{R}$, set

$$
W(\varphi, \psi)=Q_{\varphi}(\varphi, \psi)-b^{1 / 2}(Q(\varphi, \psi)) Q_{\psi}(\varphi, \psi), \quad Z(\varphi, \psi)=-Q_{\varphi}(\varphi, \psi)-b^{1 / 2}(Q(\varphi, \psi)) Q_{\psi}(\varphi, \psi) .
$$

Then, $(W, Z) \in C((0,+\infty) \times \mathbb{R}) \times C((0,+\infty) \times \mathbb{R})$ solves

$$
\begin{array}{ll}
W_{\varphi}+b^{1 / 2}(Q) W_{\psi}=\frac{1}{4} b^{-1}(Q) p(Q) W(W+Z), & (\varphi, \psi) \in(0,+\infty) \times \mathbb{R}, \\
Z_{\varphi}-b^{1 / 2}(Q) Z_{\psi}=-\frac{1}{4} b^{-1}(Q) p(Q) Z(W+Z), & (\varphi, \psi) \in(0,+\infty) \times \mathbb{R} .
\end{array}
$$

It follows from (2.66) that each characteristic is unbounded. Set

$$
h(s)=\exp \left\{\frac{1}{4} \int_{-1}^{s} b^{-1}(t) p(t) d t\right\}, \quad s<0 .
$$

It follows from (2.10) and $\gamma>1$ that

$$
\lim _{s \rightarrow 0^{-}}(-s)^{1 / 4} h(s)=0, \quad \lim _{s \rightarrow-\infty}(-s)^{1 / 2} h(s)=0 .
$$

By (2.66) and (2.69), there exists a positive constant $M_{0}$ depending only on $\gamma$ and $M$ such that

$$
b^{-1}(Q(\varphi, \psi)) p(Q(\varphi, \psi)) \geq 4 M_{0}(\varphi+1)^{-1} h(Q(\varphi, \psi)), \quad(\varphi, \psi) \in(0,+\infty) \times \mathbb{R}
$$

and

$$
b^{-3 / 2}(Q(\varphi, \psi)) p(Q(\varphi, \psi)) \geq 4 M_{0} h(Q(\varphi, \psi)), \quad(\varphi, \psi) \in(0,+\infty) \times \mathbb{R} .
$$

There are four cases to be discussed.

(i) Assume that

$$
\Psi_{+}^{\prime}(\varphi)=b^{1 / 2}\left(Q\left(\varphi, \Psi_{+}(\varphi)\right)\right), \quad \varphi>\varphi_{+}>0
$$

is a positive characteristic. Then, it follows from (2.67) that

$$
\begin{array}{r}
\left(W\left(\varphi, \Psi_{+}(\varphi)\right)\right)^{\prime}=\frac{1}{4} b^{-1}\left(Q\left(\varphi, \Psi_{+}(\varphi)\right)\right) p\left(Q\left(\varphi, \Psi_{+}(\varphi)\right)\right) W\left(\varphi, \Psi_{+}(\varphi)\right) \\
\cdot\left(W\left(\varphi, \Psi_{+}(\varphi)\right)-\left(Q\left(\varphi, \Psi_{+}(\varphi)\right)\right)^{\prime}\right), \quad \varphi>\varphi_{+}
\end{array}
$$

and thus

$$
\begin{aligned}
& \left(h\left(Q\left(\varphi, \Psi_{+}(\varphi)\right)\right) W\left(\varphi, \Psi_{+}(\varphi)\right)\right)^{\prime} \\
= & \frac{1}{4} b^{-1}\left(Q\left(\varphi, \Psi_{+}(\varphi)\right)\right) p\left(Q\left(\varphi, \Psi_{+}(\varphi)\right)\right) h\left(Q\left(\varphi, \Psi_{+}(\varphi)\right)\right) W^{2}\left(\varphi, \Psi_{+}(\varphi)\right), \quad \varphi>\varphi_{+} .
\end{aligned}
$$

Substituting (2.70) into (2.72) yields

$$
\left(h\left(Q\left(\varphi, \Psi_{+}(\varphi)\right)\right) W\left(\varphi, \Psi_{+}(\varphi)\right)\right)^{\prime} \geq M_{0}(\varphi+1)^{-1}\left(h\left(Q\left(\varphi, \Psi_{+}(\varphi)\right)\right) W\left(\varphi, \Psi_{+}(\varphi)\right)\right)^{2}, \quad \varphi>\varphi_{+},
$$


which leads to $W\left(\cdot, \Psi_{+}(\cdot)\right) \leq 0$ on $\left[\varphi_{+},+\infty\right)$.

(ii) Assume that

$$
\Psi_{-}^{\prime}(\varphi)=-b^{1 / 2}\left(Q\left(\varphi, \Psi_{-}(\varphi)\right)\right), \quad \varphi>\varphi_{-}>0
$$

is a negative characteristic. Then, it follows from (2.68) and (2.70) that

$$
\begin{aligned}
& \left(h\left(Q\left(\varphi, \Psi_{-}(\varphi)\right)\right) Z\left(\varphi, \Psi_{-}(\varphi)\right)\right)^{\prime} \\
= & -\frac{1}{4} b^{-1}\left(Q\left(\varphi, \Psi_{-}(\varphi)\right)\right) p\left(Q\left(\varphi, \Psi_{-}(\varphi)\right)\right) h\left(Q\left(\varphi, \Psi_{-}(\varphi)\right)\right) Z^{2}\left(\varphi, \Psi_{-}(\varphi)\right) \\
\leq & -M_{0}(\varphi+1)^{-1}\left(h\left(Q\left(\varphi, \Psi_{-}(\varphi)\right)\right) Z\left(\varphi, \Psi_{-}(\varphi)\right)\right)^{2}, \quad \varphi>\varphi_{-},
\end{aligned}
$$

which leads to $Z\left(\cdot, \Psi_{-}(\cdot)\right) \geq 0$ on $\left[\varphi_{-},+\infty\right)$.

(iii) Assume that

$$
\Phi_{+}^{\prime}(\psi)=b^{-1 / 2}\left(Q\left(\Phi_{+}(\psi), \psi\right)\right), \quad \psi>\psi_{+}
$$

is a positive characteristic. Then, it follows from (2.67) and (2.71) that

$$
\begin{aligned}
& \left(h\left(Q\left(\Phi_{+}(\psi), \psi\right)\right) W\left(\Phi_{+}(\psi), \psi\right)\right)^{\prime} \\
= & \frac{1}{4} b^{-3 / 2}\left(Q\left(\Phi_{+}(\psi), \psi\right)\right) p\left(Q\left(\Phi_{+}(\psi), \psi\right)\right) h\left(Q\left(\Phi_{+}(\psi), \psi\right)\right) W^{2}\left(\Phi_{+}(\psi), \psi\right) \\
\geq & M_{0}\left(h\left(Q\left(\Phi_{+}(\psi), \psi\right)\right) W\left(\Phi_{+}(\psi), \psi\right)\right)^{2}, \quad \psi>\psi_{+},
\end{aligned}
$$

which leads to $W\left(\Phi_{+}(\cdot), \cdot\right) \leq 0$ on $\left[\psi_{+},+\infty\right)$.

(iv) Assume that

$$
\Phi_{-}^{\prime}(\psi)=-b^{-1 / 2}\left(Q\left(\Phi_{-}(\psi), \psi\right)\right), \quad \psi<\psi_{-}
$$

is a negative characteristic. Then, it follows from (2.68) and (2.71) that

$$
\begin{aligned}
& \left(h\left(Q\left(\Phi_{-}(\psi), \psi\right)\right) Z\left(\Phi_{-}(\psi), \psi\right)\right)^{\prime} \\
= & -\frac{1}{4} b^{-3 / 2}\left(Q\left(\Phi_{-}(\psi), \psi\right)\right) p\left(Q\left(\Phi_{-}(\psi), \psi\right)\right) h\left(Q\left(\Phi_{-}(\psi), \psi\right)\right) Z^{2}\left(\Phi_{-}(\psi), \psi\right) \\
\leq & -M_{0}\left(h\left(Q\left(\Phi_{-}(\psi), \psi\right)\right) Z\left(\Phi_{-}(\psi), \psi\right)\right)^{2}, \quad \psi<\psi_{-},
\end{aligned}
$$

which leads to $Z\left(\Phi_{-}(\cdot), \cdot\right) \leq 0$ on $\left(-\infty, \psi_{-}\right]$.

Summing up, one gets that $W \leq 0$ and $Z \geq 0$ in $(0,+\infty) \times \mathbb{R}$.

Remark 2.7 Lemma 2.4 remains true if (2.66) is relaxed by

$$
0<-Q(\varphi, \psi)<\left\{\begin{array}{ll}
\lambda_{1}(\varphi+1)^{4 /(3-\gamma)-\lambda_{2},}, & \text { if } 1<\gamma<3, \\
\lambda_{3}(\varphi+1)^{\lambda_{4}}, & \text { if } \gamma=3, \\
+\infty, & \text { if } \gamma>3,
\end{array} \quad(\varphi, \psi) \in(0,+\infty) \times \mathbb{R},\right.
$$

where $\lambda_{i}(i=1,2,3,4)$ are positive constants with $\lambda_{2}<4 /(3-\gamma)$.

It follows from Lemmas 2.2 and 2.4 that

Proposition 2.1 Assume that $Q_{0} \in C^{1}(\mathbb{R}) \cap L^{\infty}(\mathbb{R})$ with $\sup _{\mathbb{R}} Q_{0}<0$ and $Q_{0}^{\prime} \in L^{\infty}(\mathbb{R})$, and $G_{0} \in$ $C(\mathbb{R}) \cap L^{\infty}(\mathbb{R})$. 
(i) There is not any vacuum point for a $C^{1}$ supersonic flow to the problem (2.60)-(2.62). More precisely, if $Q \in C^{1}(\mathbb{R} \times[0, \zeta])$ is a local solution to the problem (2.60) (2.62) for some positive constant $\zeta$, then

$$
-Q_{\varphi}(\varphi, \psi) \leq \max \left\{0, \sup _{\mathbb{R}}\left(-G_{0}+b^{1 / 2}\left(Q_{0}\right)\left|Q_{0}^{\prime}\right|\right)\right\}, \quad(\varphi, \psi) \in \mathbb{R} \times[0, \zeta] .
$$

(ii) If $\sup G_{0} \leq 0$, then $C^{1}$ supersonic flows to the problem (2.60)-(2.62) are uniformly away from the sonic state. More precisely, if $Q \in C^{1}(\mathbb{R} \times[0, \zeta])$ is a local solution to the problem (2.60) -(2.62) for some positive constant $\zeta$, then $\sup _{\mathbb{R} \times(0, \zeta)} Q=\sup _{\mathbb{R}} Q_{0}$.

(iii) If $\sup _{\mathbb{R}}\left(G_{0}+b^{1 / 2}\left(Q_{0}\right)\left|Q_{0}^{\prime}\right|\right)>0$, then there is not a global $C^{1}$ solution to the problem (2.60)(2.62). In particular, if $\sup _{\mathbb{R}} G_{0} \leq 0$ additionally, then there must be a shock for the global supersonic flow to the problem (2.60)-(2.62) and the flow is uniformly away from the sonic and vacuum state before the shock forms.

Remark 2.8 $\sup _{\mathbb{R}} G_{0} \leq 0$ is equivalent to that $\theta(0, \cdot)$ is nondecreasing in $\mathbb{R}$.

Remark 2.9 Proposition 2.1 shows that $\sup _{\mathbb{R}}\left(G_{0}+b^{1 / 2}\left(Q_{0}\right)\left|Q_{0}^{\prime}\right|\right) \leq 0$ is a necessary condition for the existence of global $C^{1}$ solutions to the problem (2.60)-(2.62). As will be shown in Theorem 3.4, it is also a sufficient condition.

Applying Proposition 2.1 to the supersonic flow problem in a straight nozzle gives the following theorem.

Theorem 2.3 Assume that $f \in C^{2}\left(\left[l_{0},+\infty\right)\right)$ satisfies

$$
f\left(l_{0}\right)>0, \quad f^{\prime}\left(l_{0}\right) \geq 0, \quad f^{\prime \prime}(x)=0 \text { for each } x \geq l_{0},
$$

$\Upsilon \in C^{2}\left(\left[0, f\left(l_{0}\right)\right]\right)$ satisfies (2.36) and $q_{0} \in C^{1}\left(\left[0, f\left(l_{0}\right)\right]\right)$ satisfy (2.37) and (2.39).

(i) A smooth supersonic flow to the problem (2.31) -2.35) never approaches the sonic and vacuum state in any bounded region. In particular, the speed of a smooth supersonic flow is always not less than $\inf _{\left(0, f\left(l_{0}\right)\right)} q_{0}$.

(ii) If (2.38) is invalid, then there is not a global smooth supersonic flow to the problem (2.31)(2.35). More precisely, there must be a shock for the global supersonic flow to the problem (2.31) -(2.35) and the flow is uniformly away from the sonic and vacuum state before the shock forms.

Remark 2.10 Under the assumptions of Theorem 2.3, (2.38) is necessary for the existence of global smooth supersonic flows. As will be shown in Theorem 3.3, it is also a sufficient condition. Furthermore, there is not any sonic and vacuum point for such global supersonic flows.

\subsection{On the global smooth transonic flow problem}

As a direct application of the results on the smooth supersonic flow problem (2.31)-(2.35), one can get the global extension of the smooth transonic flow obtained in Theorems 2.1] and 2.2. Assume that $f$ is a function defined on $\left[l_{-}, l_{1}\right)\left(l_{-}<0<l_{1} \leq+\infty\right)$ satisfying $\lim _{x \rightarrow l_{1}^{-}}(x+f(x))=+\infty$. Let $\Omega$ be the unbounded domain bounded by the upper wall $\Gamma_{\mathrm{up}}: y=f(x)\left(l_{-} \leq x<l_{1}\right)$, the lower wall $x$-axis and 
the inlet $\Gamma_{\text {in }}: x=g(y)\left(0 \leq y \leq f\left(l_{-}\right), g\left(f\left(l_{-}\right)\right)=_{-}\right)$. Then, the global smooth transonic problem is formulated as follows

$$
\begin{array}{ll}
\operatorname{div}\left(\rho\left(|\nabla \varphi|^{2}\right) \nabla \varphi\right)=0, & (x, y) \in \Omega, \\
\varphi(g(y), y)=C_{\text {in }}, & 0<y<f\left(l_{-}\right), \\
\rho\left(|\nabla \varphi(x, 0)|^{2}\right) \frac{\partial \varphi}{\partial y}(x, 0)=0, & g(0)<x<l_{1}, \\
\rho\left(|\nabla \varphi(x, f(x))|^{2}\right)\left(\frac{\partial \varphi}{\partial y}(x, f(x))-f^{\prime}(x) \frac{\partial \varphi}{\partial x}(x, f(x))\right)=0, & l_{-}<x<l_{1}, \\
\varphi(0, y)=0,|\nabla \varphi(0, y)|=c_{*}, & 0<y<f(0), \\
|\nabla \varphi(x, y)|<c_{*}, & (x, y) \in \Omega \text { and } x<0, \\
|\nabla \varphi(x, y)|>c_{*}, & (x, y) \in \Omega \text { and } x>0,
\end{array}
$$

where $C_{\text {in }}$ is a free constant.

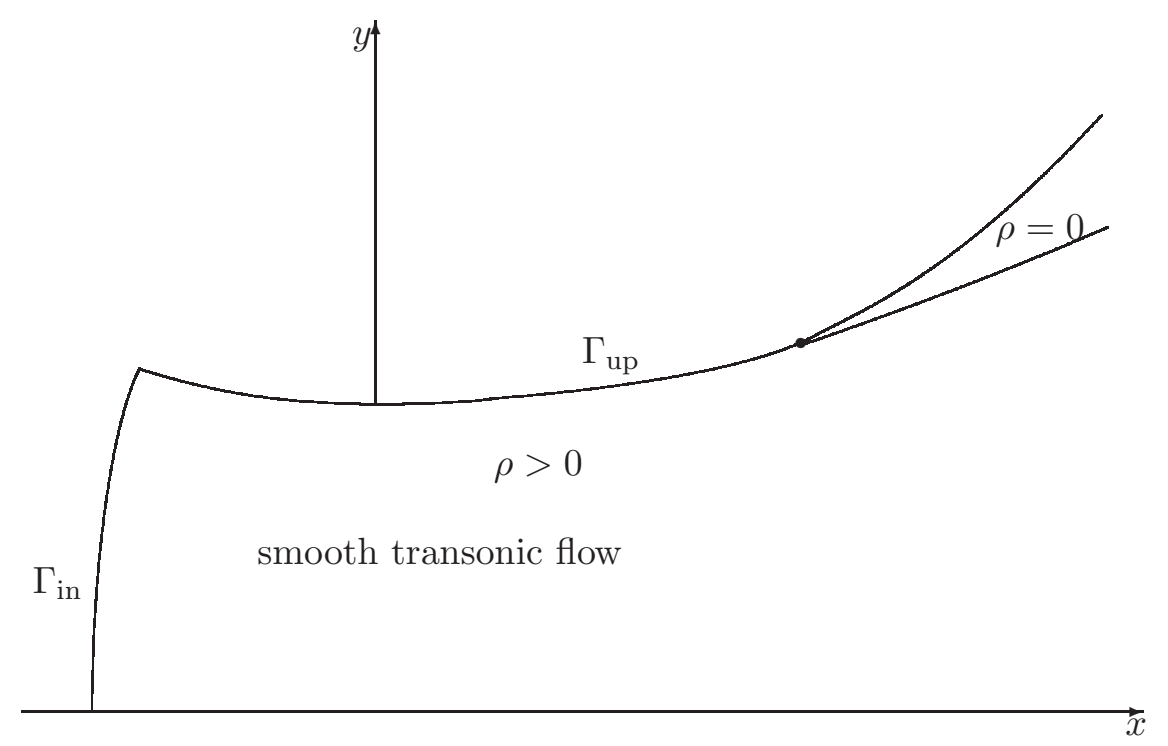

For the readers' convenience, we state the main results for the problem (2.74)-(2.80). The first one is the following existence and uniqueness.

Theorem 2.4 Assume that $f \in C^{4}\left(\left[l_{-}, l_{+}\right]\right) \cap C^{2}\left(\left[l_{-}, l_{1}\right)\right)\left(-\delta_{0,-} \leq l_{-}<0<l_{+}<l_{1} \leq+\infty\right)$ and $g \in C^{3, \alpha}\left(\left[0, f\left(l_{-}\right)\right]\right)(0<\alpha<1)$ satisfy the assumptions of Theorem 2.1 and

$$
\lim _{x \rightarrow l_{1}^{-}}(x+f(x))=+\infty, \quad f^{\prime \prime}(x) \geq 0 \text { for each } l_{+} \leq x<l_{1} .
$$

Then the problem (2.74) -(2.80) admits uniquely a global smooth solution $\varphi \in C^{1}(\bar{\Omega})$ with $|\nabla \varphi| \in$ $C^{0,1}(\bar{\Omega})$, which possesses the properties of Theorem [2.1 near the sonic state. Moreover, the global smooth transonic flow belongs to one and only one of the following two cases:

Case I Global smooth transonic flow without vacuum. In this case, $\varphi \in C^{2}(\bar{\Omega})$ and $|\nabla \varphi|<c^{*}$ on $\bar{\Omega}$. If $f^{\prime \prime}\left(1+\left(f^{\prime}\right)^{2}\right)^{-3 / 2} \in L^{\infty}\left(l_{+}, l_{1}\right)$ additionally, then $|\nabla \varphi|$ is globally Lipschitz continuous in $\Omega$.

Case II Global smooth transonic flow with vacuum. In this case, set

$$
x_{0}=\sup \left\{l \in\left(0, l_{1}\right):|\nabla \varphi(x, f(x))|<c^{*} \text { for each } 0<x<l\right\}
$$


and

$$
\Omega_{\mathrm{v}}=\left\{(x, y) \in \Omega: x>x_{0}, y>f\left(x_{0}\right)+f^{\prime}\left(x_{0}\right)\left(x-x_{0}\right)\right\} .
$$

Then

(i) $\bar{\Omega}_{\mathrm{V}}$ is the set of vacuum points. Moreover, $\varphi \in C^{2}\left(\bar{\Omega} \backslash \bar{\Omega}_{\mathrm{V}}\right)$ and

$$
\frac{\partial \varphi}{\partial x}(x, y)=\frac{c^{*}}{\sqrt{1+\left(f^{\prime}\left(x_{0}\right)\right)^{2}}}, \quad \frac{\partial \varphi}{\partial y}(x, y)=\frac{f^{\prime}\left(x_{0}\right) c^{*}}{\sqrt{1+\left(f^{\prime}\left(x_{0}\right)\right)^{2}}}, \quad(x, y) \in \partial \Omega_{\mathrm{v}} \cap \Omega .
$$

(ii) $|\nabla \varphi(x, f(x))|=c^{*}-O\left(\left(x_{0}-x\right)^{2}\right)$ as $x \rightarrow x_{0}^{-}$.

(iii) $|\nabla \varphi|$ is globally Lipschitz continuous in $\Omega$.

(iv) $|\nabla \varphi| \in C^{1}\left(\bar{\Omega} \backslash\left(x_{0}, f\left(x_{0}\right)\right)\right)$ and for each $(\tilde{x}, \tilde{y}) \in \partial \Omega_{\mathrm{v}} \cap \Omega$,

$$
\lim _{\substack{(x, y) \rightarrow(\tilde{x}, \tilde{y}) \\(x, y) \in \Omega \backslash \bar{\Omega}_{\mathrm{V}}}} \nabla|\nabla \varphi(x, y)|=(0,0), \quad \lim _{\substack{(x, y) \rightarrow(\tilde{x}, \tilde{y}) \\(x, y) \in \Omega \backslash \Omega_{\mathrm{V}}}}\left(c^{*}-|\nabla \varphi(x, y)|\right)^{1 / 2} \nabla \arctan \frac{\varphi_{y}(x, y)}{\varphi_{x}(x, y)}=(0,0) .
$$

In particular, if $1<\gamma \leq 2$, then $\rho\left(|\nabla \varphi|^{2}\right) \nabla \varphi \in C^{1}\left(\bar{\Omega} \backslash\left(x_{0}, f\left(x_{0}\right)\right)\right)$.

(v) If $1<\gamma<3$ and $f^{\prime \prime}\left(x_{0}\right)>0$ additionally, then for any $\varepsilon>0$,

$$
\lim _{\substack{(x, y) \rightarrow(\tilde{x}, \tilde{y}) \\(x, y) \in \Omega \backslash \Omega_{\mathrm{v}}}}\left(c^{*}-|\nabla \varphi(x, y)|\right)^{-(\gamma+1+\varepsilon) /(4 \gamma-4)} \nabla|\nabla \varphi(x, y)| \cdot\left(-1, f^{\prime}\left(x_{0}\right)\right)=+\infty, \quad(\tilde{x}, \tilde{y}) \in \partial \Omega_{\mathrm{V}} \cap \Omega .
$$

Remark 2.11 If $f \in C^{2,1}\left(\left[l_{+}, l_{1}\right)\right)$ additionally, then the acceleration of the flow is Lipschitz continuous in the gas region.

The following theorem gives some sufficient conditions to determine whether a vacuum forms or not for global smooth transonic flows.

Theorem 2.5 (i) Set

$$
\check{x}=\inf \left\{x \in\left(0, l_{1}\right): \arctan f^{\prime}(x) \geq \int_{-\infty}^{h(x)} b^{1 / 2}(s) d s\right\},
$$

where

$$
h(x)=-\frac{1}{f(0) c_{*}^{2 /(\gamma-1)}} \int_{0}^{x} \arctan f^{\prime}(s) \sqrt{1+\left(f^{\prime}(s)\right)^{2}} d s, \quad x \in\left(0, l_{1}\right) .
$$

Then there is no vacuum on $\left\{(x, f(x)): l_{-} \leq x \leq \check{x}\right\}$ for the global smooth transonic flow to the problem (2.74)-(2.80). If

$$
f^{\prime \prime}(x)=0, \quad x>\check{x}
$$

then there is no vacuum for the global smooth transonic flow to the problem (2.74) -(2.80).

(ii) If $\arctan f^{\prime}\left(l_{1}\right) \geq \int_{-\infty}^{0} b^{1 / 2}(s) d s$, then the global smooth transonic flow to the problem (2.74) (2.80) admits a vacuum. Furthermore, the first vacuum point in the increasing $x$-direction must form at the upper wall before

$$
\hat{x}=\inf \left\{x \in\left(0, l_{1}\right): \arctan f^{\prime}(x) \geq \int_{-\infty}^{0} b^{1 / 2}(s) d s\right\} .
$$

(iii) If

$$
\begin{cases}\lim _{x \rightarrow+\infty} f^{\prime \prime}(x) x^{2 \gamma /(\gamma+1)}=+\infty, & \text { when } l_{1}=+\infty \text { and } f^{\prime}(+\infty)<+\infty, \\ \lim _{x \rightarrow+l_{1}} \frac{f^{\prime \prime}(x)}{\left(f^{\prime}(x)\right)^{3}} f^{2 \gamma /(\gamma+1)}(x)=+\infty, & \text { when } l_{1} \leq+\infty \text { and } f^{\prime}\left(l_{1}\right)=+\infty,\end{cases}
$$

then the global smooth transonic flow to the problem (2.74)-(2.80) admits a vacuum. 


\section{Smooth supersonic flows before vacuum formation}

In this section, we consider the smooth supersonic flow problem (2.31)-(2.35) before vacuum formation. For the existence, we will solve the problem (2.40)-(2.45), which is equivalent to the problem (2.31)(2.35) when there is no vacuum, by a fixed point argument. Since (2.40) is singular at the vacuum, a cut-off technique is needed. For $0<\varepsilon<1 / \mu_{2}$, let $H_{\varepsilon} \in C^{\infty}(\mathbb{R})$ be a cut-off function such that

$$
H_{\varepsilon}(s)\left\{\begin{array}{ll}
=s, & \text { if } s \geq-\frac{1}{\varepsilon}, \\
=-\frac{2}{\varepsilon}, & \text { if } s \leq-\frac{2}{\varepsilon}
\end{array} \quad \text { and } \quad 0 \leq H_{\varepsilon}^{\prime}(s) \leq 2 \text { for } s \in \mathbb{R} .\right.
$$

\subsection{Semilinear problems}

Give $\mathscr{Q} \in C\left(\left[l_{0}, l_{1}\right)\right)$ satisfying

$$
c_{*} \leq \mathscr{Q}(x) \leq c^{*}, \quad l_{0}<x<l_{1} .
$$

Fix $\zeta>0$. Assume that $\tilde{Q} \in C^{0,1}([0, \zeta] \times[0, m])$ satisfies

$$
\tilde{Q}(\varphi, \psi) \leq-\mu_{1}, \quad(\varphi, \psi) \in(0, \zeta) \times(0, m)
$$

and

$$
-\beta \leq \tilde{Q}_{\varphi}(\varphi, \psi) \leq 0, \quad\left|\tilde{Q}_{\psi}(\varphi, \psi)\right| \leq \beta, \quad(\varphi, \psi) \in(0, \zeta) \times(0, m)
$$

where $\beta$ is a positive constant to be determined. Define

$$
\begin{array}{ll}
\tilde{W}(\varphi, \psi)=\frac{\partial H_{\varepsilon}(\tilde{Q})}{\partial \varphi}(\varphi, \psi)-b^{1 / 2}\left(H_{\varepsilon}(\tilde{Q}(\varphi, \psi)) \frac{\partial H_{\varepsilon}(\tilde{Q})}{\partial \psi}(\varphi, \psi),\right. & (\varphi, \psi) \in(0, \zeta) \times(0, m), \\
\tilde{Z}(\varphi, \psi)=-\frac{\partial H_{\varepsilon}(\tilde{Q})}{\partial \varphi}(\varphi, \psi)-b^{1 / 2}\left(H_{\varepsilon}(\tilde{Q}(\varphi, \psi))\right) \frac{\partial H_{\varepsilon}(\tilde{Q})}{\partial \psi}(\varphi, \psi), & (\varphi, \psi) \in(0, \zeta) \times(0, m) .
\end{array}
$$

By (3.2), (3.3) and the definition of $H_{\varepsilon}$, there exists a positive constant $M_{1}=M_{1}\left(\gamma, \mu_{1}\right)$ such that

$$
|\tilde{W}(\varphi, \psi)| \leq M_{1} \beta, \quad|\tilde{Z}(\varphi, \psi)| \leq M_{1} \beta, \quad(\varphi, \psi) \in(0, \zeta) \times(0, m) .
$$

Here and hereafter, a parenthesis after a generic constant means that this constant depends only on the variables in the parentheses. Consider the problem

$$
\begin{array}{ll}
W_{\varphi}+b^{1 / 2}\left(H_{\varepsilon}(\tilde{Q})\right) W_{\psi}=\frac{1}{4} b^{-1}\left(H_{\varepsilon}(\tilde{Q})\right) p\left(H_{\varepsilon}(\tilde{Q})\right) W(W+\tilde{Z}), & (\varphi, \psi) \in(0, \zeta) \times(0, m), \\
Z_{\varphi}-b^{1 / 2}\left(H_{\varepsilon}(\tilde{Q})\right) Z_{\psi}=-\frac{1}{4} b^{-1}\left(H_{\varepsilon}(\tilde{Q})\right) p\left(H_{\varepsilon}(\tilde{Q})\right) Z(\tilde{W}+Z), & (\varphi, \psi) \in(0, \zeta) \times(0, m), \\
W(0, \psi)=W_{0}(\psi), & \psi \in(0, m), \\
Z(0, \psi)=Z_{0}(\psi), & \psi \in(0, m), \\
W(\varphi, 0)+Z(\varphi, 0)=0, & \varphi \in(0, \zeta), \\
W(\varphi, m)+Z(\varphi, m)=\tilde{h}(\varphi), & \varphi \in(0, \zeta), \\
Q_{\varphi}=\frac{1}{2}(W-Z), & (\varphi, \psi) \in(0, \zeta) \times(0, m), \\
Q(0, \psi)=Q_{0}(\psi), & \psi \in(0, m),
\end{array}
$$


where

$$
\tilde{h}(\varphi)=\frac{2 f^{\prime \prime}\left(X_{\mathrm{up}}(\varphi)\right)\left(1+\left(f^{\prime}\left(X_{\mathrm{up}}(\varphi)\right)\right)^{2}\right)^{-3 / 2}}{b^{1 / 2}\left(H_{\varepsilon}(\tilde{Q}(\varphi, m))\right) A_{+}^{-1}(\tilde{Q}(\varphi, m))}, \quad \varphi \in(0, \zeta) .
$$

It follows from (2.30), (3.2) and the definition of $H_{\varepsilon}$ that there exists a positive constant $M_{2}=$ $M_{2}(\gamma, \zeta, \varepsilon, f)$ such that

$$
0 \leq \tilde{h}(\varphi) \leq M_{2}, \quad \varphi \in(0, \zeta)
$$

Indeed, $M_{2}$ depends only on $\gamma, \varepsilon$ and $\sup _{\left(l_{0}, X_{\text {up }}(\zeta)\right)} f^{\prime \prime}\left(1+\left(f^{\prime}\right)^{2}\right)^{-3 / 2}$.

Lemma 3.1 Assume that $W_{0}, Z_{0} \in L^{\infty}(0, m)$ satisfy (2.59). Then the problem (3.5)-(3.10) admits a unique weak solution $(W, Z) \in L^{\infty}((0, \zeta) \times(0, m)) \times L^{\infty}((0, \zeta) \times(0, m))$. Furthermore, the solution satisfies

$$
-\mu_{4}\left(1+\mu_{3}\right) \leq W(\varphi, \psi) \leq 0, \quad 0 \leq Z(\varphi, \psi) \leq \mu_{4}\left(1+\mu_{3}\right), \quad(\varphi, \psi) \in(0, \zeta) \times(0, m),
$$

where $\mu_{4}=\mu_{4}\left(\gamma, m, \mu_{1}, \zeta, \varepsilon, f\right)$ is a positive constant.

Proof. Since the system (3.5), (3.6) is strictly hyperbolic according to (3.2) and the definition of $H_{\varepsilon}$, the classical theory yields the local existence and the uniqueness of the weak solution to the problem (3.5)-(3.10). By (2.59) and (3.13), one can get the global existence. Furthermore, the solution satisfies

$$
W(\varphi, \psi) \leq 0, \quad Z(\varphi, \psi) \geq 0, \quad(\varphi, \psi) \in(0, \zeta) \times(0, m)
$$

Assume that

$$
\Sigma_{ \pm}: \Psi_{ \pm}^{\prime}(\varphi)= \pm b^{1 / 2}\left(H_{\varepsilon}\left(\tilde{Q}\left(\varphi, \Psi_{ \pm}(\varphi)\right)\right)\right), \quad 0<\Psi_{ \pm}(\varphi)<m, \quad \hat{\varphi}_{ \pm}<\varphi<\check{\varphi}_{ \pm} \quad\left(0 \leq \hat{\varphi}_{ \pm}<\check{\varphi}_{ \pm} \leq \zeta\right)
$$

are a positive and a negative characteristics, respectively. By (3.5) and (3.6), $W$ and $Z$ satisfy

$$
\frac{d}{d \varphi} W\left(\varphi, \Psi_{+}(\varphi)\right) \geq \tilde{R}_{+}(\varphi) W\left(\varphi, \Psi_{+}(\varphi)\right), \quad \hat{\varphi}_{+}<\varphi<\check{\varphi}_{+}
$$

and

$$
\frac{d}{d \varphi} Z\left(\varphi, \Psi_{-}(\varphi)\right) \leq \tilde{R}_{-}(\varphi) Z\left(\varphi, \Psi_{-}(\varphi)\right), \quad \hat{\varphi}_{-}<\varphi<\check{\varphi}_{-}
$$

on $\Sigma_{ \pm}$, respectively, where

$$
\tilde{R}_{ \pm}(\varphi)=\frac{d}{d \varphi} F\left(H_{\varepsilon}\left(\tilde{Q}\left(\varphi, \Psi_{ \pm}(\varphi)\right)\right)\right), \quad \varphi \in\left(\hat{\varphi}_{ \pm}, \check{\varphi}_{ \pm}\right)
$$

with

$$
F(s)=\frac{1}{4} \int_{s}^{-1} b^{-1}(t) p(t) d t, \quad s<0 .
$$

If follows from (3.15)-(3.18) that

$$
W\left(\check{\varphi}, \Psi_{+}(\check{\varphi})\right) \geq M_{3} W\left(\hat{\varphi}, \Psi_{+}(\hat{\varphi})\right), \quad Z\left(\check{\varphi}, \Psi_{-}(\check{\varphi})\right) \leq M_{3} Z\left(\hat{\varphi}, \Psi_{-}(\hat{\varphi})\right)
$$


with $M_{3}=\exp \left\{F(-2 / \varepsilon)-F\left(-\mu_{1}\right)\right\}>1$ depending only on $\gamma, \mu_{1}$ and $\varepsilon$.

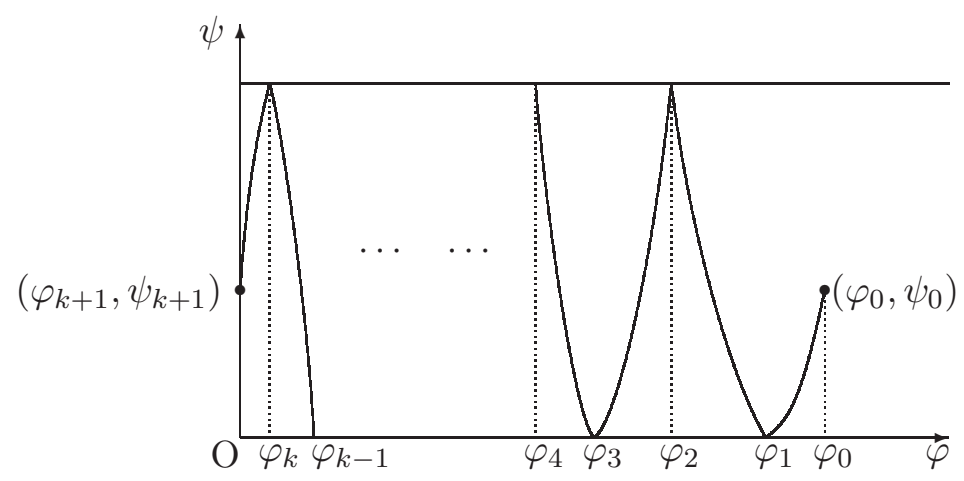

the case that $k$ is even

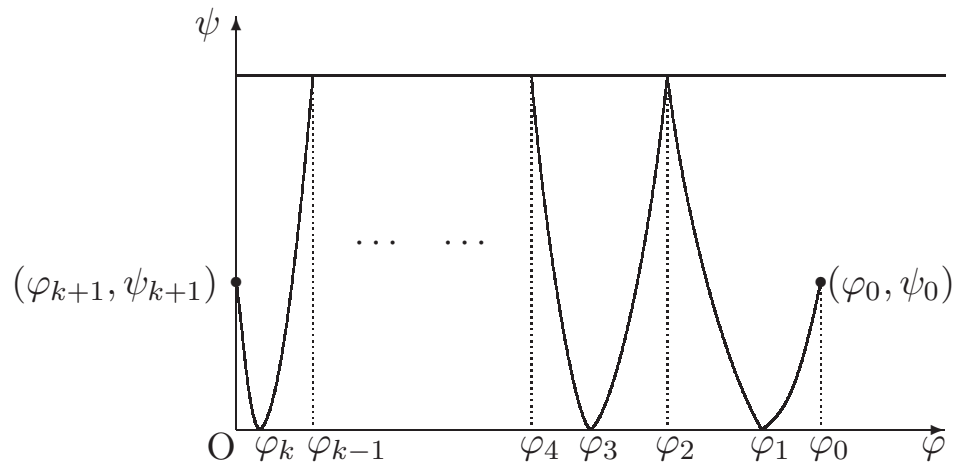

the case that $k$ is odd

We now estimate the lower bound of $W$ by the method of characteristics. Fix $\left(\varphi_{0}, \psi_{0}\right) \in(0, \zeta) \times$ $(0, m)$. Let $\psi=\Psi_{1}(\varphi)$ be the positive characteristic from $\left(\varphi_{0}, \psi_{0}\right)$, which approaches either $\{0\} \times[0, m]$ or $(0, \zeta) \times\{0\}$ at a point $\left(\varphi_{1}, \psi_{1}\right)$. If $\varphi_{1}>0$, then there exists a negative characteristic $\psi=\Psi_{2}(\varphi)$ from $\left(\varphi_{1}, \psi_{1}\right)$, which approaches either $\{0\} \times[0, m]$ or $(0, \zeta) \times\{m\}$ at a point $\left(\varphi_{2}, \psi_{2}\right)$. If $\varphi_{2}>0$, then there exists a positive characteristic $\psi=\Psi_{3}(\varphi)$ from $\left(\varphi_{2}, \psi_{2}\right)$, which approaches either $\{0\} \times[0, m]$ or $(0, \zeta) \times\{0\}$ at a point $\left(\varphi_{3}, \psi_{3}\right)$. Therefore, there exists a nonnegative integer $k$ such that

$$
\begin{gathered}
\varphi_{0}>\varphi_{1}>\cdots>\varphi_{k}>\varphi_{k+1}=0, \\
\psi_{j}= \begin{cases}0, & 1 \leq j \leq k \text { and } j \text { is odd, } \quad 0 \leq \psi_{k+1} \leq m, \\
m, & 1 \leq j \leq k \text { and } j \text { is even, }\end{cases} \\
\left\{\begin{array}{l}
\Psi_{j}^{\prime}(\varphi)=(-1)^{j-1} b^{1 / 2}\left(H_{\varepsilon}\left(\tilde{Q}\left(\varphi, \Psi_{j}(\varphi)\right)\right)\right), \quad \varphi_{j}<\varphi<\varphi_{j-1}, \quad 1 \leq j \leq k+1 . \\
\Psi_{j}\left(\varphi_{j}\right)=\psi_{j}, \quad \Psi_{j}\left(\varphi_{j-1}\right)=\psi_{j-1},
\end{array}\right.
\end{gathered}
$$

By (3.2), there exists an integer $K=K\left(\gamma, m, \mu_{1}, \zeta\right)$ such that $k \leq K$. For $1 \leq j \leq k+1$, it follows from (3.19) that

$$
W\left(\varphi_{j-1}, \psi_{j-1}\right) \geq M_{3} W\left(\varphi_{j}, \psi_{j}\right) \text { if } j \text { is odd, } \quad Z\left(\varphi_{j-1}, \psi_{j-1}\right) \leq M_{3} Z\left(\varphi_{j}, \psi_{j}\right) \text { if } j \text { is even. }
$$

If $k$ is even, one can get from (3.20), (3.7)-(3.10) that

$$
W\left(\varphi_{0}, \psi_{0}\right) \geq M_{3} W\left(\varphi_{1}, \psi_{1}\right)=-M_{3} Z\left(\varphi_{1}, \psi_{1}\right)
$$




$$
\begin{aligned}
& \geq-M_{3}^{2} Z\left(\varphi_{2}, \psi_{2}\right)=M_{3}^{2} W\left(\varphi_{2}, \psi_{2}\right)-M_{3}^{2} \tilde{h}\left(\varphi_{2}, \psi_{2}\right) \\
& \geq M_{3}^{k+1} W_{0}\left(\psi_{k+1}\right)-\sum_{2 \leq 2 j \leq k} M_{3}^{2 j} \tilde{h}\left(\varphi_{2 j}, \psi_{2 j}\right) .
\end{aligned}
$$

Similarly, if $k$ is odd, then

$$
W\left(\varphi_{0}, \psi_{0}\right) \geq-M_{3}^{k+1} Z_{0}\left(\psi_{k+1}\right)-\sum_{2 \leq 2 j \leq k} M_{3}^{2 j} \tilde{h}\left(\varphi_{2 j}, \psi_{2 j}\right) .
$$

It follows from (3.21), (3.22), (2.59) and (3.13) that in both cases,

$$
W\left(\varphi_{0}, \psi_{0}\right) \geq-\mu_{3} M_{3}^{K+1}-K M_{3}^{K} M_{2},
$$

which yields the lower bound of $W$ in (3.14) by choosing $\mu_{4}=M_{3}^{K}\left(M_{3}+K M_{2}\right)$. The upper bound of $Z$ in (3.14) can be obtained similarly.

Lemma 3.2 Let $(W, Z) \in L^{\infty}((0, \zeta) \times(0, m)) \times L^{\infty}((0, \zeta) \times(0, m))$ be the weak solution to the problem (3.5) -(3.10) with $W_{0}, Z_{0} \in L^{\infty}(0, m)$ satisfying (2.59). Assume that $Q_{0} \in L^{\infty}(0, m)$ satisfies (2.58) and

$$
Q_{0}(\psi) \geq-\frac{1}{\varepsilon}, \quad 0<\psi<m
$$

Then the problem (3.11), (3.12) admits a unique weak solution $Q \in L^{\infty}((0, \zeta) \times(0, m))$. Furthermore, the solution $Q \in C^{0,1}([0, \zeta] \times[0, m])$ and it satisfies

$$
\begin{gathered}
-\mu_{2}-\mu_{4}\left(1+\mu_{3}\right) \zeta \leq Q(\varphi, \psi) \leq-\mu_{1}, \quad(\varphi, \psi) \in(0, \zeta) \times(0, m), \\
-\mu_{4}\left(1+\mu_{3}\right) \leq Q_{\varphi}(\varphi, \psi) \leq 0, \quad(\varphi, \psi) \in(0, \zeta) \times(0, m), \\
\left|Q_{\psi}(\varphi, \psi)\right| \leq \mu_{5}\left(1+\mu_{3}^{2}\right)(1+\beta \varphi), \quad(\varphi, \psi) \in(0, \zeta) \times(0, m),
\end{gathered}
$$

where $\mu_{5}=\mu_{5}\left(\gamma, m, \mu_{1}, \zeta, \varepsilon, f\right)$ is a positive constant.

Proof. The existence and uniqueness of the weak solution to the problem (3.11), (3.12) are clear. Moreover, (3.25) follows from (3.11) and (3.14), while (3.24) follows from (2.58) and (3.25), directly. It remains to prove (3.26). Rewrite (3.5) as

$$
W_{\psi}=-b^{-1 / 2}\left(H_{\varepsilon}(\tilde{Q})\right) W_{\varphi}+\frac{1}{4} b^{-3 / 2}\left(H_{\varepsilon}(\tilde{Q})\right) p\left(H_{\varepsilon}(\tilde{Q})\right) W(W+\tilde{Z}), \quad(\varphi, \psi) \in(0, \zeta) \times(0, m) .
$$

Fix $\hat{\varphi} \in(0, \zeta)$ and $\psi \in(0, m)$. Integrating (3.27) over $(0, \hat{\varphi})$, one gets from (3.3), (3.4), (3.14) and the definition of $H_{\varepsilon}$ that

$$
\begin{aligned}
& \left|\frac{d}{d \psi} \int_{0}^{\hat{\varphi}} W(\varphi, \psi) d \varphi\right| \\
=\mid-b^{-1 / 2}\left(H_{\varepsilon}(\tilde{Q}(\hat{\varphi}, \psi))\right) W(\hat{\varphi}, \psi)+b^{-1 / 2}\left(H_{\varepsilon}(\tilde{Q}(0, \psi))\right) W(0, \psi) & \\
& \quad-\frac{1}{2} \int_{0}^{\hat{\varphi}} b^{-3 / 2}\left(H_{\varepsilon}(\tilde{Q}(\varphi, \psi))\right) p\left(H_{\varepsilon}(\tilde{Q}(\varphi, \psi))\right) H_{\varepsilon}^{\prime}(\tilde{Q}(\varphi, \psi)) \tilde{Q}_{\varphi}(\varphi, \psi) W(\varphi, \psi) d \varphi \\
& +\frac{1}{4} \int_{0}^{\hat{\varphi}} b^{-3 / 2}\left(H_{\varepsilon}(\tilde{Q}(\varphi, \psi))\right) p\left(H_{\varepsilon}(\tilde{Q}(\varphi, \psi))\right) W(\varphi, \psi)(W(\varphi, \psi)+\tilde{Z}(\varphi, \psi)) d \varphi \mid \\
\leq & \mu_{4}\left(1+\mu_{3}\right)\left(b^{-1 / 2}\left(H_{\varepsilon}(\tilde{Q}(\hat{\varphi}, \psi))\right)+b^{-1 / 2}\left(H_{\varepsilon}(\tilde{Q}(0, \psi))\right)\right)
\end{aligned}
$$




$$
\begin{aligned}
&-\frac{1}{2} \mu_{4}\left(1+\mu_{3}\right) \int_{0}^{\hat{\varphi}} b^{-3 / 2}\left(H_{\varepsilon}(\tilde{Q}(\varphi, \psi))\right) p\left(H_{\varepsilon}(\tilde{Q}(\varphi, \psi))\right) H_{\varepsilon}^{\prime}(\tilde{Q}(\varphi, \psi)) \tilde{Q}_{\varphi}(\varphi, \psi) d \varphi \\
&+\frac{1}{4} \mu_{4}\left(1+\mu_{3}\right) \int_{0}^{\hat{\varphi}} b^{-3 / 2}\left(H_{\varepsilon}(\tilde{Q}(\varphi, \psi))\right) p\left(H_{\varepsilon}(\tilde{Q}(\varphi, \psi))\right)(|W(\varphi, \psi)|+|\tilde{Z}(\varphi, \psi)|) d \varphi \\
& \leq \mu_{4}\left(1+\mu_{3}\right)\left(b^{-1 / 2}\left(H_{\varepsilon}(\tilde{Q}(\hat{\varphi}, \psi))\right)+b^{-1 / 2}\left(H_{\varepsilon}(\tilde{Q}(0, \psi))\right)\right) \\
&+\mu_{4}\left(1+\mu_{3}\right)\left(b^{-1 / 2}\left(H_{\varepsilon}(\tilde{Q}(\hat{\varphi}, \psi))\right)-b^{-1 / 2}\left(H_{\varepsilon}(\tilde{Q}(0, \psi))\right)\right) \\
&+\frac{1}{4} \mu_{4}\left(1+\mu_{3}\right)\left(\mu_{4}\left(1+\mu_{3}\right)+M_{1} \beta\right) \int_{0}^{\hat{\varphi}} b^{-3 / 2}\left(H_{\varepsilon}(\tilde{Q}(\varphi, \psi))\right) p\left(H_{\varepsilon}(\tilde{Q}(\varphi, \psi))\right) d \varphi .
\end{aligned}
$$

A similar argument shows

$$
\begin{aligned}
& \quad\left|\frac{d}{d \psi} \int_{0}^{\hat{\varphi}} Z(\varphi, \psi) d \varphi\right| \\
& \leq \mu_{4}\left(1+\mu_{3}\right)\left(b^{-1 / 2}\left(H_{\varepsilon}(\tilde{Q}(\hat{\varphi}, \psi))\right)+b^{-1 / 2}\left(H_{\varepsilon}(\tilde{Q}(0, \psi))\right)\right) \\
& \quad+\mu_{4}\left(1+\mu_{3}\right)\left(b^{-1 / 2}\left(H_{\varepsilon}(\tilde{Q}(\hat{\varphi}, \psi))\right)-b^{-1 / 2}\left(H_{\varepsilon}(\tilde{Q}(0, \psi))\right)\right) \\
& \quad+\frac{1}{4} \mu_{4}\left(1+\mu_{3}\right)\left(\mu_{4}\left(1+\mu_{3}\right)+M_{1} \beta\right) \int_{0}^{\hat{\varphi}} b^{-3 / 2}\left(H_{\varepsilon}(\tilde{Q}(\varphi, \psi))\right) p\left(H_{\varepsilon}(\tilde{Q}(\varphi, \psi))\right) d \varphi .
\end{aligned}
$$

It follows from (3.23) and (2.59) that

$$
\left|Q_{0}^{\prime}(\psi)\right| \leq M_{4} \mu_{3}
$$

where $M_{4}=M_{4}(\gamma, \varepsilon)$ is a positive constant. By (3.11), 3.28)-(3.30), there exists a positive constant $\mu_{5}=\mu_{5}\left(\gamma, m, \mu_{1}, \zeta, \varepsilon, f\right)$ such that

$$
\left|Q_{\psi}(\hat{\varphi}, \psi)\right|=\left|Q_{0}^{\prime}(\psi)+\frac{1}{2} \frac{d}{d \psi} \int_{0}^{\hat{\varphi}}(W(\varphi, \psi)-Z(\varphi, \psi)) d \varphi\right| \leq \mu_{5}\left(1+\mu_{3}^{2}\right)(1+\beta \hat{\varphi}),
$$

which implies (3.26).

Remark 3.1 Precisely, $\mu_{4}$ and $\mu_{5}$ depend only on $\gamma, m, \mu_{1}, \zeta, \varepsilon$ and $\sup _{\left(l_{0}, X_{\mathrm{up}}(\zeta)\right)} f^{\prime \prime}\left(1+\left(f^{\prime}\right)^{2}\right)^{-3 / 2}$.

\subsection{Existence and uniqueness}

Theorem 3.1 Assume that $f \in C^{2}\left(\left[l_{0}, l_{1}\right)\right)$ satisfies (2.30), and $Q_{0} \in C^{1}([0, m])$ and $G_{0} \in C([0, m])$ satisfy (2.47) and (2.48). Then the problem (2.40) -(2.45) admits uniquely a solution $Q \in C^{1}([0, \zeta) \times$ $[0, m])$, where either $\zeta=+\infty$ or $0<\zeta<+\infty$ with $\lim _{\varphi \rightarrow \zeta^{-}[0, m]} Q(\varphi, \cdot)=-\infty$. Furthermore, the solution satisfies

$$
-Q_{\varphi}(\varphi, \psi) \pm b^{1 / 2}(Q(\varphi, \psi)) Q_{\psi}(\varphi, \psi) \geq 0, \quad(\varphi, \psi) \in(0, \zeta) \times(0, m) .
$$

Proof. The uniqueness result follows from the uniqueness of the classical solution to the problem (2.31) -2.35), which is a standard strictly hyperbolic problem, since the problem (2.40)-(2.45) and the problem (2.31)-(2.35) are equivalent for supersonic flows without vacuum.

For the existence, we claim that for any $0<\varepsilon<1 / \mu_{2}$ and any $\hat{\zeta}>0$, there exists $\hat{\zeta}_{\varepsilon} \in(0, \hat{\zeta}]$ such that the problem (2.49) -2.57) with $\zeta=\hat{\zeta}_{\varepsilon}$ admits a weak solution $(W, Z, Q) \in L^{\infty}\left(\left(0, \hat{\zeta}_{\varepsilon}\right) \times(0, m)\right) \times$ $L^{\infty}\left(\left(0, \hat{\zeta}_{\varepsilon}\right) \times(0, m)\right) \times C^{0,1}\left(\left[0, \hat{\zeta}_{\varepsilon}\right] \times[0, m]\right)$ satisfying

$$
Q(\varphi, \psi)>-\frac{1}{\varepsilon}, \quad W(\varphi, \psi) \leq 0, \quad Z(\varphi, \psi) \geq 0, \quad(\varphi, \psi) \in\left(0, \hat{\zeta}_{\varepsilon}\right) \times(0, m)
$$


and either $\hat{\zeta}_{\varepsilon}=\hat{\zeta}$ or $0<\hat{\zeta}_{\varepsilon}<\hat{\zeta}$ with $\min _{[0, m]} Q\left(\hat{\zeta}_{\varepsilon}, \cdot\right)=-1 / \varepsilon$. Assuming the claim, we complete the proof of the theorem first. Since $\hat{\zeta}>0$ is arbitrary, there exists $0<\zeta_{\varepsilon} \leq+\infty$ such that the problem (2.49) - 2.57) with $\zeta=\zeta_{\varepsilon}$ admits a weak solution $(W, Z, Q) \in L^{\infty}\left(\left(0, \zeta_{\varepsilon}\right) \times(0, m)\right) \times L^{\infty}\left(\left(0, \zeta_{\varepsilon}\right) \times\right.$ $(0, m)) \times C^{0,1}\left(\left[0, \zeta_{\varepsilon}\right) \times[0, m]\right)$ satisfying

$$
Q(\varphi, \psi)>-\frac{1}{\varepsilon}, \quad W(\varphi, \psi) \leq 0, \quad Z(\varphi, \psi) \geq 0, \quad(\varphi, \psi) \in\left(0, \zeta_{\varepsilon}\right) \times(0, m)
$$

and either $\zeta_{\varepsilon}=+\infty$ or $0<\zeta_{\varepsilon}<+\infty$ with $\lim _{\varphi \rightarrow \zeta_{\varepsilon}^{-}} \min _{[0, m]} Q(\varphi, \cdot)=-1 / \varepsilon$. Equivalently, $Q$ solves the problem (2.40)-(2.45) with $\zeta=\zeta_{\varepsilon}$. The regularity of initial and boundary data and (2.48) yield that $Q \in C^{1}\left(\left[0, \zeta_{\varepsilon}\right) \times[0, m]\right)$. Then, the theorem follows from the arbitrariness of $\varepsilon \in\left(0,1 / \mu_{2}\right)$.

We now turn to the proof of the claim. Fix $0<\varepsilon<1 / \mu_{2}$ and $\hat{\zeta}>0$. Let $\mu_{4}$ and $\mu_{5}$ be the constants determined in Lemmas 3.1 and 3.2 with $\zeta=\hat{\zeta}$, which depend only on $\gamma, m, \mu_{1}, \hat{\zeta}, \varepsilon$ and $f$. The proof consists of four steps.

Step I Local existence.

Choose

$$
\beta=\max \left\{\mu_{4}\left(1+\mu_{3}\right), 2 \mu_{5}\left(1+\mu_{3}^{2}\right)\right\} .
$$

For $\mathscr{Q} \in C\left(\left[l_{0}, l_{1}\right)\right)$ satisfying (3.1) and $\tilde{Q} \in C^{0,1}([0, \hat{\zeta}] \times[0, m])$ satisfying (3.2) and (3.3) with $\zeta=\hat{\zeta}$ and (3.31), Lemmas 3.1 and 3.2 show that the problem (3.5) -3.12) with $\zeta=\hat{\zeta}$ admits a unique weak solution $(W, Z, Q) \in L^{\infty}((0, \hat{\zeta}) \times(0, m)) \times L^{\infty}((0, \hat{\zeta}) \times(0, m)) \times C^{0,1}([0, \hat{\zeta}] \times[0, m])$ and $(W, Z, Q)$ satisfies

$$
\begin{gathered}
-\mu_{4}\left(1+\mu_{3}\right) \leq W(\varphi, \psi) \leq 0, \quad 0 \leq Z(\varphi, \psi) \leq \mu_{4}\left(1+\mu_{3}\right), \quad(\varphi, \psi) \in(0, \hat{\zeta}) \times(0, m), \\
-\mu_{2}-\mu_{4}\left(1+\mu_{3}\right) \hat{\zeta} \leq Q(\varphi, \psi) \leq-\mu_{1}, \quad(\varphi, \psi) \in(0, \hat{\zeta}) \times(0, m), \\
-\mu_{4}\left(1+\mu_{3}\right) \leq Q_{\varphi}(\varphi, \psi) \leq 0, \quad(\varphi, \psi) \in(0, \hat{\zeta}) \times(0, m), \\
\left|Q_{\psi}(\varphi, \psi)\right| \leq \mu_{5}\left(1+\mu_{3}^{2}\right)(1+\beta \varphi), \quad(\varphi, \psi) \in(0, \hat{\zeta}) \times(0, m) .
\end{gathered}
$$

Let $\hat{\zeta}_{0}=\min \{\hat{\zeta}, 1 / \beta\}$. Then, it follows from (3.34) and (3.35) that

$$
-\beta \leq Q_{\varphi}(\varphi, \psi) \leq 0, \quad\left|Q_{\psi}(\varphi, \psi)\right| \leq \beta, \quad(\varphi, \psi) \in\left(0, \hat{\zeta}_{0}\right) \times(0, m) .
$$

Set

$$
\hat{\mathscr{Q}}(x)= \begin{cases}A_{+}^{-1}\left(Q\left(\Phi_{\mathrm{up}}(x), m\right)\right), & l_{0} \leq x \leq X_{\mathrm{up}}\left(\hat{\zeta}_{0}\right), \\ A_{+}^{-1}\left(Q\left(\hat{\zeta}_{0}, m\right)\right), & X_{\text {up }}\left(\hat{\zeta}_{0}\right)<x<l_{1},\end{cases}
$$

where $\Phi_{\text {up }}$ and $X_{\text {up }}$ are defined in $\S 2.3$. Define

$$
\begin{gathered}
\mathscr{S}_{0}=\left\{(\mathscr{Q}, Q) \in C\left(\left[l_{0}, l_{1}\right)\right) \times C^{0,1}\left(\left[0, \hat{\zeta}_{0}\right] \times[0, m]\right): c_{*} \leq \mathscr{Q}(x) \leq c^{*} \text { for } l_{0}<x<l_{1},\right. \\
-\mu_{2}-\mu_{4}\left(1+\mu_{3}\right) \hat{\zeta} \leq Q(\varphi, \psi) \leq-\mu_{1},-\beta \leq Q_{\varphi}(\varphi, \psi) \leq 0 \\
\left.\quad \text { and }\left|Q_{\psi}(\varphi, \psi)\right| \leq \beta \text { for }(\varphi, \psi) \in\left(0, \hat{\zeta}_{0}\right) \times(0, m)\right\}
\end{gathered}
$$

with the following norm

$$
\|(\mathscr{Q}, Q)\|_{\mathscr{S}_{0}}=\max \left\{\|\mathscr{Q}\|_{L^{\infty}\left(l_{0}, l_{1}\right)},\|Q\|_{L^{\infty}\left(\left(0, \hat{\zeta}_{0}\right) \times(0, m)\right)}\right\}, \quad(\mathscr{Q}, Q) \in \mathscr{S}_{0} .
$$


By (3.33) and (3.36),$(\hat{\mathscr{Q}}, Q) \in \mathscr{S}_{0}$. Therefore, one can define a mapping as follows

$$
\mathscr{J}_{0}: \mathscr{S}_{0} \longrightarrow \mathscr{S}_{0}, \quad(\mathscr{Q}, \tilde{Q}) \longmapsto(\hat{\mathcal{Q}}, Q) .
$$

By (3.36), $\mathscr{J}_{0}$ is compact. Furthermore, one can show that $\mathscr{J}_{0}$ is continuous (see for example [10] Theorem 5.1). Then, it follows from the Schauder fixed point theorem that $\mathscr{J}_{0}$ admits a fixed point $(\mathscr{Q}, Q)$. That is to say, there exists $(W, Z, Q) \in L^{\infty}\left(\left(0, \hat{\zeta}_{0}\right) \times(0, m)\right) \times L^{\infty}\left(\left(0, \hat{\zeta}_{0}\right) \times(0, m)\right) \times C^{0,1}\left(\left[0, \hat{\zeta}_{0}\right] \times\right.$ $[0, m])$, which solves

$$
\begin{aligned}
& W_{\varphi}+b^{1 / 2}\left(H_{\varepsilon}(Q)\right) W_{\psi}=\frac{1}{4} b^{-1}\left(H_{\varepsilon}(Q)\right) p\left(H_{\varepsilon}(Q)\right) W\left(W-H_{\varepsilon}^{\prime}(Q) Q_{\varphi}\right. \\
& \left.-b^{1 / 2}\left(H_{\varepsilon}(Q)\right) H_{\varepsilon}^{\prime}(Q) Q_{\psi}\right), \quad(\varphi, \psi) \in\left(0, \hat{\zeta}_{0}\right) \times(0, m), \\
& Z_{\varphi}-b^{1 / 2}\left(H_{\varepsilon}(Q)\right) Z_{\psi}=-\frac{1}{4} b^{-1}\left(H_{\varepsilon}(Q)\right) p\left(H_{\varepsilon}(Q)\right) Z\left(H_{\varepsilon}^{\prime}(Q) Q_{\varphi}\right. \\
& \left.-b^{1 / 2}\left(H_{\varepsilon}(Q)\right) H_{\varepsilon}^{\prime}(Q) Q_{\psi}+Z\right), \quad(\varphi, \psi) \in\left(0, \hat{\zeta}_{0}\right) \times(0, m), \\
& W(0, \psi)=W_{0}(\psi), \\
& \psi \in(0, m) \text {, } \\
& Z(0, \psi)=Z_{0}(\psi) \text {, } \\
& \psi \in(0, m) \text {, } \\
& W(\varphi, 0)+Z(\varphi, 0)=0 \text {, } \\
& W(\varphi, m)+Z(\varphi, m)=\frac{2 f^{\prime \prime}\left(X_{\mathrm{up}}(\varphi)\right)\left(1+\left(f^{\prime}\left(X_{\mathrm{up}}(\varphi)\right)\right)^{2}\right)^{-3 / 2}}{b^{1 / 2}\left(H_{\varepsilon}(Q(\varphi, m))\right) A_{+}^{-1}(Q(\varphi, m))}, \quad \varphi \in\left(0, \hat{\zeta}_{0}\right), \\
& Q_{\varphi}=\frac{1}{2}(W-Z), \\
& (\varphi, \psi) \in\left(0, \hat{\zeta}_{0}\right) \times(0, m), \\
& Q(0, \psi)=Q_{0}(\psi), \\
& \psi \in(0, m), \\
& \mathscr{Q}(x)=A_{+}^{-1}\left(Q\left(\Phi_{\text {up }}(x), m\right)\right), \\
& x \in\left(l_{0}, X_{\text {up }}\left(\hat{\zeta}_{0}\right)\right) \text {. }
\end{aligned}
$$

Furthermore, (3.32) and (3.33) lead to

$$
\begin{aligned}
-\mu_{4}\left(1+\mu_{3}\right) & \leq W(\varphi, \psi) \leq 0, \quad 0 \leq Z(\varphi, \psi) \leq \mu_{4}\left(1+\mu_{3}\right), \quad(\varphi, \psi) \in\left(0, \hat{\zeta}_{0}\right) \times(0, m), \\
& -\mu_{2}-\mu_{4}\left(1+\mu_{3}\right) \hat{\zeta} \leq Q(\varphi, \psi) \leq-\mu_{1}, \quad(\varphi, \psi) \in\left(0, \hat{\zeta}_{0}\right) \times(0, m) .
\end{aligned}
$$

Let

$$
\hat{\zeta}_{0, \varepsilon}=\sup \left\{\tilde{\varphi} \in\left(0, \hat{\zeta}_{0}\right): Q(\varphi, \psi)>-\frac{1}{\varepsilon} \text { for each }(\varphi, \psi) \in(0, \tilde{\varphi}) \times(0, m)\right\} .
$$

Then, for $(\varphi, \psi) \in\left(0, \hat{\zeta}_{0, \varepsilon}\right) \times(0, m)$,

$$
W(\varphi, \psi)=Q_{\varphi}(\varphi, \psi)-b^{1 / 2}(Q(\varphi, \psi)) Q_{\psi}(\varphi, \psi), \quad Z(\varphi, \psi)=-Q_{\varphi}(\varphi, \psi)-b^{1 / 2}(Q(\varphi, \psi)) Q_{\psi}(\varphi, \psi),
$$

and $(W, Z, Q)$ solves

$$
\begin{array}{ll}
W_{\varphi}+b^{1 / 2}(Q) W_{\psi}=\frac{1}{4} b^{-1}(Q) p(Q) W(W+Z), & (\varphi, \psi) \in\left(0, \hat{\zeta}_{0, \varepsilon}\right) \times(0, m), \\
Z_{\varphi}-b^{1 / 2}(Q) Z_{\psi}=-\frac{1}{4} b^{-1}(Q) p(Q) Z(W+Z), & (\varphi, \psi) \in\left(0, \hat{\zeta}_{0, \varepsilon}\right) \times(0, m), \\
W(0, \psi)=W_{0}(\psi), & \psi \in(0, m), \\
Z(0, \psi)=Z_{0}(\psi), & \psi \in(0, m), \\
W(\varphi, 0)+Z(\varphi, 0)=0, & \varphi \in\left(0, \hat{\zeta}_{0, \varepsilon}\right), \\
W(\varphi, m)+Z(\varphi, m)=\frac{2 f^{\prime \prime}\left(X_{\mathrm{up}}(\varphi)\right)\left(1+\left(f^{\prime}\left(X_{\mathrm{up}}(\varphi)\right)\right)^{2}\right)^{-3 / 2}}{b^{1 / 2}(Q(\varphi, m)) A_{+}^{-1}(Q(\varphi, m))}, & \varphi \in\left(0, \hat{\zeta}_{0, \varepsilon}\right),
\end{array}
$$




$$
\begin{array}{ll}
Q_{\varphi}=\frac{1}{2}(W-Z), & (\varphi, \psi) \in\left(0, \hat{\zeta}_{0, \varepsilon}\right) \times(0, m), \\
Q(0, \psi)=Q_{0}(\psi), & \psi \in(0, m), \\
\mathscr{Q}(x)=A_{+}^{-1}\left(Q\left(\Phi_{\mathrm{up}}(x), m\right)\right), & x \in\left(l_{0}, X_{\mathrm{up}}\left(\hat{\zeta}_{0, \varepsilon}\right)\right) .
\end{array}
$$

It follows from (3.37) and (3.38) that

$$
\begin{gathered}
-\mu_{4}\left(1+\mu_{3}\right) \leq W(\varphi, \psi) \leq 0, \quad 0 \leq Z(\varphi, \psi) \leq \mu_{4}\left(1+\mu_{3}\right), \quad(\varphi, \psi) \in\left(0, \hat{\zeta}_{0, \varepsilon}\right) \times(0, m), \\
-\mu_{2}-\mu_{4}\left(1+\mu_{3}\right) \hat{\zeta} \leq Q(\varphi, \psi) \leq-\mu_{1}, \quad(\varphi, \psi) \in\left(0, \hat{\zeta}_{0, \varepsilon}\right) \times(0, m) .
\end{gathered}
$$

If $\hat{\zeta}_{0, \varepsilon}=\hat{\zeta}$ or $\min _{[0, m]} Q\left(\hat{\zeta}_{0, \varepsilon}, \cdot\right)=-1 / \varepsilon$, then the claim is proved. So, without loss of generality, it is assumed that $\hat{\zeta}_{0, \varepsilon}<\hat{\zeta}$ and $\min _{[0, m]} Q\left(\hat{\zeta}_{0, \varepsilon}, \cdot\right)>-1 / \varepsilon$, which also leads to $\hat{\zeta}_{0, \varepsilon}=\hat{\zeta}_{0}$.

Step II Extension.

Set

$$
\hat{\mu}_{2}=\mu_{2}+\mu_{4}\left(1+\mu_{3}\right) \hat{\zeta}, \quad \hat{\mu}_{3}=\mu_{4}\left(1+\mu_{3}\right), \quad \hat{\beta}=\max \left\{\mu_{4}\left(1+\hat{\mu}_{3}\right), 2 \mu_{5}\left(1+\hat{\mu}_{3}^{2}\right)\right\} .
$$

Let $\hat{\zeta}_{1}=\min \left\{\hat{\zeta}, \hat{\zeta}_{0}+1 / \hat{\beta}\right\}$. Define

$$
\begin{gathered}
\mathscr{S}_{1}=\left\{(\mathscr{Q}, Q) \in C\left(\left[X_{\text {up }}\left(\hat{\zeta}_{0}\right), l_{1}\right)\right) \times C^{0,1}\left(\left[\hat{\zeta}_{0}, \hat{\zeta}_{1}\right] \times[0, m]\right): c_{*} \leq \mathscr{Q}(x) \leq c^{*} \text { for } X_{\text {up }}\left(\hat{\zeta}_{0}\right)<x<l_{1},\right. \\
-\hat{\mu}_{2}-\mu_{4}\left(1+\hat{\mu}_{3}\right) \hat{\zeta} \leq Q(\varphi, \psi) \leq-\mu_{1},-\hat{\beta} \leq Q_{\varphi}(\varphi, \psi) \leq 0 \\
\left.\quad \text { and }\left|Q_{\psi}(\varphi, \psi)\right| \leq \hat{\beta} \text { for }(\varphi, \psi) \in\left(\hat{\zeta}_{0}, \hat{\zeta}_{1}\right) \times(0, m)\right\} .
\end{gathered}
$$

For given $(\mathscr{Q}, \tilde{Q}) \in \mathscr{S}_{1}$, consider the problem

$$
\begin{array}{ll}
W_{\varphi}+b^{1 / 2}\left(H_{\varepsilon}(\tilde{Q})\right) W_{\psi}=\frac{1}{4} b^{-1}\left(H_{\varepsilon}(\tilde{Q})\right) p\left(H_{\varepsilon}(\tilde{Q})\right) W(W+\tilde{Z}), & (\varphi, \psi) \in\left(\hat{\zeta}_{0}, \hat{\zeta}_{1}\right) \times(0, m), \\
Z_{\varphi}-b^{1 / 2}\left(H_{\varepsilon}(\tilde{Q})\right) Z_{\psi}=-\frac{1}{4} b^{-1}\left(H_{\varepsilon}(\tilde{Q})\right) p\left(H_{\varepsilon}(\tilde{Q})\right) Z(\tilde{W}+Z), & (\varphi, \psi) \in\left(\hat{\zeta}_{0}, \hat{\zeta}_{1}\right) \times(0, m), \\
W\left(\hat{\zeta}_{0}, \psi\right)=W_{1}(\psi), & \psi \in(0, m), \\
Z\left(\hat{\zeta}_{0}, \psi\right)=Z_{1}(\psi), & \psi \in(0, m), \\
W(\varphi, 0)+Z(\varphi, 0)=0, & \varphi \in\left(\hat{\zeta}_{0}, \hat{\zeta}_{1}\right), \\
W(\varphi, m)+Z(\varphi, m)=\tilde{h}(\varphi), & \varphi \in\left(\hat{\zeta}_{0}, \hat{\zeta}_{1}\right), \\
Q_{\varphi}=\frac{1}{2}(W-Z), & (\varphi, \psi) \in\left(\hat{\zeta}_{0}, \hat{\zeta}_{1}\right) \times(0, m), \\
Q\left(\hat{\zeta}_{0}, \psi\right)=Q_{1}(\psi), & \psi \in(0, m),
\end{array}
$$

where

$$
\begin{array}{ll}
\tilde{W}(\varphi, \psi)=\frac{\partial H_{\varepsilon}(\tilde{Q})}{\partial \varphi}(\varphi, \psi)-b^{1 / 2}\left(H_{\varepsilon}(\tilde{Q}(\varphi, \psi)) \frac{\partial H_{\varepsilon}(\tilde{Q})}{\partial \psi}(\varphi, \psi),\right. & (\varphi, \psi) \in\left(\hat{\zeta}_{0}, \hat{\zeta}_{1}\right) \times(0, m), \\
\tilde{Z}(\varphi, \psi)=-\frac{\partial H_{\varepsilon}(\tilde{Q})}{\partial \varphi}(\varphi, \psi)-b^{1 / 2}\left(H_{\varepsilon}(\tilde{Q}(\varphi, \psi))\right) \frac{\partial H_{\varepsilon}(\tilde{Q})}{\partial \psi}(\varphi, \psi), & (\varphi, \psi) \in\left(\hat{\zeta}_{0}, \hat{\zeta}_{1}\right) \times(0, m)
\end{array}
$$

and

$$
W_{1}(\psi)=W\left(\hat{\zeta}_{0}, \psi\right), \quad Z_{1}(\psi)=Z\left(\hat{\zeta}_{0}, \psi\right), \quad Q_{1}(\psi)=Q\left(\hat{\zeta}_{0}, \psi\right), \quad \psi \in(0, m)
$$


It follows from (3.39) and (3.40) that $Q_{1} \in C^{0,1}([0, m]), W_{1}, Z_{1} \in L^{\infty}(0, m)$ such that

$$
-\hat{\mu}_{2} \leq Q_{1}(\psi) \leq-\mu_{1}, \quad-\hat{\mu}_{3} \leq W_{1}(\psi) \leq 0, \quad 0 \leq Z_{1}(\psi) \leq \hat{\mu}_{3}, \quad \psi \in(0, m) .
$$

Then, Lemmas 3.1 and 3.2 show that the problem (3.41) admits a unique weak solution $(W, Z, Q) \in L^{\infty}\left(\left(\hat{\zeta}_{0}, \hat{\zeta}_{1}\right) \times(0, m)\right) \times L^{\infty}\left(\left(\hat{\zeta}_{0}, \hat{\zeta}_{1}\right) \times(0, m)\right) \times C^{0,1}\left(\left[\hat{\zeta}_{0}, \hat{\zeta}_{1}\right] \times[0, m]\right)$ and it satisfies

$$
\begin{gathered}
-\mu_{4}\left(1+\hat{\mu}_{3}\right) \leq W(\varphi, \psi) \leq 0, \quad 0 \leq Z(\varphi, \psi) \leq \mu_{4}\left(1+\hat{\mu}_{3}\right), \quad(\varphi, \psi) \in\left(\hat{\zeta}_{0}, \hat{\zeta}_{1}\right) \times(0, m), \\
-\hat{\mu}_{2}-\mu_{4}\left(1+\hat{\mu}_{3}\right) \hat{\zeta} \leq Q(\varphi, \psi) \leq-\mu_{1}, \quad(\varphi, \psi) \in\left(\hat{\zeta}_{0}, \hat{\zeta}_{1}\right) \times(0, m), \\
-\hat{\beta} \leq-\mu_{4}\left(1+\hat{\mu}_{3}\right) \leq Q_{\varphi}(\varphi, \psi) \leq 0, \quad(\varphi, \psi) \in\left(\hat{\zeta}_{0}, \hat{\zeta}_{1}\right) \times(0, m), \\
\left|Q_{\psi}(\varphi, \psi)\right| \leq \mu_{5}\left(1+\hat{\mu}_{3}^{2}\right)\left(1+\hat{\beta}\left(\varphi-\hat{\zeta}_{0}\right)\right) \leq \hat{\beta}, \quad(\varphi, \psi) \in\left(\hat{\zeta}_{0}, \hat{\zeta}_{1}\right) \times(0, m) .
\end{gathered}
$$

Therefore, one can define a mapping as follows

$$
\mathscr{J}_{1}: \mathscr{S}_{1} \longrightarrow \mathscr{S}_{1}, \quad(\mathscr{Q}, \tilde{Q}) \longmapsto(\hat{\mathscr{Q}}, Q),
$$

where

$$
\hat{\mathscr{Q}}(x)= \begin{cases}A_{+}^{-1}\left(Q\left(\Phi_{\text {up }}(x), m\right)\right), & X_{\text {up }}\left(\hat{\zeta}_{0}\right) \leq x \leq X_{\text {up }}\left(\hat{\zeta}_{1}\right), \\ A_{+}^{-1}\left(Q\left(\hat{\zeta}_{1}, m\right)\right), & X_{\text {up }}\left(\hat{\zeta}_{1}\right)<x<l_{1} .\end{cases}
$$

Then, the Schauder fixed point theorem shows that $\mathscr{J}_{1}$ admits a fixed point $(\mathscr{Q}, Q)$. That is to say, there exists $(W, Z, Q) \in L^{\infty}\left(\left(\hat{\zeta}_{0}, \hat{\zeta}_{1}\right) \times(0, m)\right) \times L^{\infty}\left(\left(\hat{\zeta}_{0}, \hat{\zeta}_{1}\right) \times(0, m)\right) \times C^{0,1}\left(\left[\hat{\zeta}_{0}, \hat{\zeta}_{1}\right] \times[0, m]\right)$ satisfying (3.50) -3.53 and solving

$$
\begin{array}{ll}
W_{\varphi}+b^{1 / 2}\left(H_{\varepsilon}(Q)\right) W_{\psi}=\frac{1}{4} b^{-1}\left(H_{\varepsilon}(Q)\right) p\left(H_{\varepsilon}(Q)\right) W\left(W-H_{\varepsilon}^{\prime}(Q) Q_{\varphi}\right. & \\
Z_{\varphi}-b^{1 / 2}\left(H_{\varepsilon}(Q)\right) Z_{\psi}=-\frac{1}{4} b^{-1}\left(H_{\varepsilon}(Q)\right) p\left(H_{\varepsilon}(Q)\right) Z\left(H_{\varepsilon}^{\prime}(Q) Q_{\varphi}\right. & (\varphi, \psi) \in\left(\hat{\zeta}_{0}, \hat{\zeta}_{1}\right) \times(0, m), \\
\left.-b^{1 / 2}\left(H_{\varepsilon}(Q)\right) H_{\varepsilon}^{\prime}(Q) Q_{\psi}+Z\right), & (\varphi, \psi) \in\left(\hat{\zeta}_{0}, \hat{\zeta}_{1}\right) \times(0, m), \\
W\left(\hat{\zeta}_{0}, \psi\right)=W_{1}(\psi), & \psi \in(0, m), \\
Z\left(\hat{\zeta}_{0}, \psi\right)=Z_{1}(\psi), & \psi \in(0, m), \\
W(\varphi, 0)+Z(\varphi, 0)=0, & \varphi \in\left(\hat{\zeta}_{0}, \hat{\zeta}_{1}\right), \\
W(\varphi, m)+Z(\varphi, m)=\frac{2 f^{\prime \prime}\left(X_{\mathrm{up}}(\varphi)\right)\left(1+\left(f^{\prime}\left(X_{\mathrm{up}}(\varphi)\right)\right)^{2}\right)^{-3 / 2}}{b^{1 / 2}\left(H_{\varepsilon}(Q(\varphi, m))\right) A_{+}^{-1}(Q(\varphi, m))}, & \varphi \in\left(\hat{\zeta}_{0}, \hat{\zeta}_{1}\right), \\
Q \varphi=\frac{1}{2}(W-Z), & (\varphi, \psi) \in\left(\hat{\zeta}_{0}, \hat{\zeta}_{1}\right) \times(0, m), \\
Q\left(\hat{\zeta}_{0}, \psi\right)=Q_{1}(\psi), & \psi \in(0, m), \\
\mathscr{Q}(x)=A_{+}^{-1}\left(Q\left(\Phi_{\mathrm{up}}(x), m\right)\right), & x \in\left(X_{\mathrm{up}}\left(\hat{\zeta}_{0}\right), X_{\mathrm{up}}\left(\hat{\zeta}_{1}\right)\right) .
\end{array}
$$

Let

$$
\hat{\zeta}_{1, \varepsilon}=\sup \left\{\tilde{\varphi} \in\left(\hat{\zeta}_{0}, \hat{\zeta}_{1}\right): Q(\varphi, \psi)>-\frac{1}{\varepsilon} \text { for each }(\varphi, \psi) \in\left(\hat{\zeta}_{0}, \tilde{\varphi}\right) \times(0, m)\right\} .
$$

Then, $(W, Z, Q)$ solves

$$
W_{\varphi}+b^{1 / 2}(Q) W_{\psi}=\frac{1}{4} b^{-1}(Q) p(Q) W(W+Z), \quad(\varphi, \psi) \in\left(\hat{\zeta}_{0}, \hat{\zeta}_{1, \varepsilon}\right) \times(0, m)
$$




$$
\begin{array}{ll}
Z_{\varphi}-b^{1 / 2}(Q) Z_{\psi}=-\frac{1}{4} b^{-1}(Q) p(Q) Z(W+Z), & (\varphi, \psi) \in\left(\hat{\zeta}_{0}, \hat{\zeta}_{1, \varepsilon}\right) \times(0, m), \\
W\left(\hat{\zeta}_{0}, \psi\right)=W_{1}(\psi), & \psi \in(0, m), \\
Z\left(\hat{\zeta}_{0}, \psi\right)=Z_{1}(\psi), & \psi \in(0, m), \\
W(\varphi, 0)+Z(\varphi, 0)=0, & \varphi \in\left(\hat{\zeta}_{0}, \hat{\zeta}_{1, \varepsilon}\right), \\
W(\varphi, m)+Z(\varphi, m)=\frac{2 f^{\prime \prime}\left(X_{\mathrm{up}}(\varphi)\right)\left(1+\left(f^{\prime}\left(X_{\mathrm{up}}(\varphi)\right)\right)^{2}\right)^{-3 / 2}}{b^{1 / 2}(Q(\varphi, m)) A_{+}^{-1}(Q(\varphi, m))}, & \varphi \in\left(\hat{\zeta}_{0}, \hat{\zeta}_{1, \varepsilon}\right), \\
Q_{\varphi}=\frac{1}{2}(W-Z), & (\varphi, \psi) \in\left(\hat{\zeta}_{0}, \hat{\zeta}_{1, \varepsilon}\right) \times(0, m), \\
Q\left(\hat{\zeta}_{0}, \psi\right)=Q_{1}(\psi), & \psi \in(0, m), \\
\mathscr{Q}(x)=A_{+}^{-1}\left(Q\left(\Phi_{\mathrm{up}}(x), m\right)\right), & x \in\left(X_{\mathrm{up}}\left(\hat{\zeta}_{0}\right), X_{\mathrm{up}}\left(\hat{\zeta}_{1, \varepsilon}\right)\right) .
\end{array}
$$

Combining the above existence result and Step I shows that $(W, Z, Q) \in L^{\infty}\left(\left(0, \hat{\zeta}_{1, \varepsilon}\right) \times(0, m)\right) \times$ $L^{\infty}\left(\left(0, \hat{\zeta}_{1, \varepsilon}\right) \times(0, m)\right) \times C^{0,1}\left(\left[0, \hat{\zeta}_{1, \varepsilon}\right] \times[0, m]\right)$, which satisfies

$$
-\frac{1}{\varepsilon}<Q(\varphi, \psi) \leq-\mu_{1}, \quad-Q_{\varphi}(\varphi, \psi) \pm b^{1 / 2}(Q(\varphi, \psi)) Q_{\psi}(\varphi, \psi) \geq 0, \quad(\varphi, \psi) \in\left(0, \hat{\zeta}_{1, \varepsilon}\right) \times(0, m)
$$

and solves

$$
\begin{array}{ll}
W_{\varphi}+b^{1 / 2}(Q) W_{\psi}=\frac{1}{4} b^{-1}(Q) p(Q) W(W+Z), & (\varphi, \psi) \in\left(0, \hat{\zeta}_{1, \varepsilon}\right) \times(0, m), \\
Z_{\varphi}-b^{1 / 2}(Q) Z_{\psi}=-\frac{1}{4} b^{-1}(Q) p(Q) Z(W+Z), & (\varphi, \psi) \in\left(0, \hat{\zeta}_{1, \varepsilon}\right) \times(0, m), \\
W(0, \psi)=W_{0}(\psi), & \psi \in(0, m), \\
Z(0, \psi)=Z_{0}(\psi), & \psi \in(0, m), \\
W(\varphi, 0)+Z(\varphi, 0)=0, & \varphi \in\left(0, \hat{\zeta}_{1, \varepsilon}\right), \\
W(\varphi, m)+Z(\varphi, m)=\frac{2 f^{\prime \prime}\left(X_{\mathrm{up}}(\varphi)\right)\left(1+\left(f^{\prime}\left(X_{\mathrm{up}}(\varphi)\right)\right)^{2}\right)^{-3 / 2}}{b^{1 / 2}(Q(\varphi, m)) A_{+}^{-1}(Q(\varphi, m))}, & \varphi \in\left(0, \hat{\zeta}_{1, \varepsilon}\right), \\
Q & (\varphi, \psi) \in\left(0, \hat{\zeta}_{1, \varepsilon}\right) \times(0, m) \\
Q(0, \psi)=Q_{0}(\psi), & \psi \in(0, m), \\
\mathscr{Q}(x)=A_{+}^{-1}\left(Q\left(\Phi_{\mathrm{up}}(x), m\right)\right), & x \in\left(l_{0}, X_{\mathrm{up}}\left(\hat{\zeta}_{1, \varepsilon}\right)\right) .
\end{array}
$$

Step III Estimate.

Note that $(W, Z)$ solves the problem (3.55)-(3.60) with $Q$ satisfying (3.54). Then, Lemma 3.1 shows that $W$ and $Z$ satisfy

$$
-\mu_{4}\left(1+\mu_{3}\right) \leq W(\varphi, \psi) \leq 0, \quad 0 \leq Z(\varphi, \psi) \leq \mu_{4}\left(1+\mu_{3}\right), \quad(\varphi, \psi) \in\left(0, \hat{\zeta}_{1, \varepsilon}\right) \times(0, m),
$$

which, together with (3.61), (3.62) and (2.47), also leads to

$$
-\mu_{2}-\mu_{4}\left(1+\mu_{3}\right) \hat{\zeta} \leq Q(\varphi, \psi) \leq-\mu_{1}, \quad(\varphi, \psi) \in\left(0, \hat{\zeta}_{1, \varepsilon}\right) \times(0, m) .
$$

Step IV Iteration.

If $\hat{\zeta}_{1, \varepsilon}=\hat{\zeta}$ or $\min _{[0, m]} Q\left(\hat{\zeta}_{1, \varepsilon}, \cdot\right)=-1 / \varepsilon$, then the claim is proved. Otherwise, $\hat{\zeta}_{1, \varepsilon}<\hat{\zeta}$ and $\min _{[0, m]} Q\left(\hat{\zeta}_{1, \varepsilon}, \cdot\right)>$ $-1 / \varepsilon$, which also leads to $\hat{\zeta}_{1, \varepsilon}=\hat{\zeta}_{1}$. Set

$$
W_{2}(\psi)=W\left(\hat{\zeta}_{1}, \psi\right), \quad Z_{2}(\psi)=Z\left(\hat{\zeta}_{1}, \psi\right), \quad Q_{2}(\psi)=Q\left(\hat{\zeta}_{1}, \psi\right), \quad \psi \in(0, m) .
$$


It follows from (3.63) and (3.64) that $Q_{2} \in C^{0,1}([0, m])$ and $W_{2}, Z_{2} \in L^{\infty}(0, m)$ satisfy

$$
-\hat{\mu}_{2} \leq Q_{2}(\psi) \leq-\mu_{1}, \quad-\hat{\mu}_{3} \leq W_{2}(\psi) \leq 0, \quad 0 \leq Z_{2}(\psi) \leq \hat{\mu}_{3}, \quad \psi \in(0, m) .
$$

Note that $\left(W_{2}, Z_{2}, Q_{2}\right)$ satisfies the same condition as (3.49) for $\left(W_{1}, Z_{1}, Q_{1}\right)$. Then, repeating Step II, one gets $\hat{\zeta}_{2, \varepsilon} \in\left(\zeta_{1}, \hat{\zeta}\right]$ such that the problem (2.49)-(2.57) with $\zeta=\hat{\zeta}_{2, \varepsilon}$ admits a weak solution $(W, Z, Q) \in L^{\infty}\left(\left(0, \hat{\zeta}_{2, \varepsilon}\right) \times(0, m)\right) \times L^{\infty}\left(\left(0, \hat{\zeta}_{2, \varepsilon}\right) \times(0, m)\right) \times C^{0,1}\left(\left[0, \hat{\zeta}_{2, \varepsilon}\right] \times[0, m]\right)$ satisfying

$$
-\frac{1}{\varepsilon}<Q(\varphi, \psi) \leq-\mu_{1}, \quad-Q_{\varphi}(\varphi, \psi) \pm b^{1 / 2}(Q(\varphi, \psi)) Q_{\psi}(\varphi, \psi) \geq 0, \quad(\varphi, \psi) \in\left(0, \hat{\zeta}_{2, \varepsilon}\right) \times(0, m)
$$

and either $\hat{\zeta}_{2, \varepsilon}=\min \left\{\hat{\zeta}, \hat{\zeta}_{0}+2 / \hat{\beta}\right\}$ or $\hat{\zeta}_{2, \varepsilon}<\min \left\{\hat{\zeta}, \hat{\zeta}_{0}+2 / \hat{\beta}\right\}$ with $\min _{[0, m]} Q\left(\hat{\zeta}_{2, \varepsilon}, \cdot\right)=-1 / \varepsilon$. Then, $\hat{\zeta}_{\varepsilon}$ in the claim can be obtained after repeating Step III and Step II for a finite number of times.

Remark 3.2 The estimates in Lemmas 3.1 and 3.2 are independent of the value of the solution in the domain. So, the solution to the problem (3.5)-(3.12) can satisfy the same estimates at each $\varphi$, which guarantees the success of the iteration in the proof of Theorem [3.1.

Remark 3.3 The iteration in the proof of Theorem 3.1 begins from not $\left(W_{1}, Z_{1}, Q_{1}\right)$ but $\left(W_{2}, Z_{2}, Q_{2}\right)$. Indeed, the conditions between $\left(W_{1}, Z_{1}, Q_{1}\right)$ and $\left(W_{0}, Z_{0}, Q_{0}\right)$ are different, while $\left(W_{2}, Z_{2}, Q_{2}\right)$ satisfies the same conditions as for $\left(W_{1}, Z_{1}, Q_{1}\right)$. Note that the estimates in Lemmas 3.1 and 3.2 depend on the initial data. To show that $\left(W_{2}, Z_{2}, Q_{2}\right)$ satisfies the same conditions as for $\left(W_{1}, Z_{1}, Q_{1}\right)$, the initial data of $\left(W_{2}, Z_{2}, Q_{2}\right)$ is taken to be not $\left(W_{1}, Z_{1}, Q_{1}\right)$ but $\left(W_{0}, Z_{0}, Q_{0}\right)$ (see Step III).

Remark 3.4 It should be noted that for the local existence of sonic-supersonic flows in [10], the iteration scheme in the fixed point argument is

$$
\begin{aligned}
& W_{\varphi}+b^{1 / 2}(\tilde{Q}) W_{\psi}=\frac{1}{4} b^{-1}(\tilde{Q}) p(\tilde{Q}) \tilde{W}(W+Z), \\
& Z_{\varphi}-b^{1 / 2}(\tilde{Q}) Z_{\psi}=-\frac{1}{4} b^{-1}(\tilde{Q}) p(\tilde{Q}) \tilde{Z}(W+Z),
\end{aligned}
$$

where

$$
\tilde{W}=\tilde{Q}_{\varphi}-b^{1 / 2}(\tilde{Q}) \tilde{Q}_{\psi}, \quad \tilde{Z}=-\tilde{Q}_{\varphi}-b^{1 / 2}(\tilde{Q}) \tilde{Q}_{\psi} .
$$

This iteration does not work for Theorem [3.1. The reason lies in that the desired estimates in [10] need $\tilde{W} \leq 0$ and $\tilde{Z} \geq 0$, while the set of $\tilde{Q}$ satisfying $\tilde{W} \leq 0$ and $\tilde{Z} \geq 0$ is not convex. Furthermore, another disadvantage is that in Theorem 3.1 the boundary condition at $\psi=m$ may be large.

In the physical plane, Theorem 3.1 can be stated as follows.

Theorem 3.2 Assume that $f \in C^{2}\left(\left[l_{0}, l_{1}\right)\right)$ satisfies (2.30), $\Upsilon \in C^{2}\left(\left[0, f\left(l_{0}\right)\right]\right)$ satisfies (2.36) and $q_{0} \in C^{1}\left(\left[0, f\left(l_{0}\right)\right]\right)$ satisfies (2.37) -(2.39). Then the problem (2.31)-(2.35) admits uniquely a maximal smooth supersonic flow before vacuum formation. Furthermore, the solution satisfies one and only one of the following two cases:

(i) $\varphi \in C^{2}(\bar{\Omega})$ and $c_{1} \leq|\nabla \varphi|<c^{*}$ on $\bar{\Omega}$.

(ii) $\varphi \in C^{2}\left(\bar{\Omega}_{\zeta} \backslash \Gamma_{\zeta}\right)$ with

$$
c_{1} \leq|\nabla \varphi(x, y)|<c^{*} \text { for each }(x, y) \in \bar{\Omega}_{\zeta} \backslash \Gamma_{\zeta}, \quad \sup _{\Omega_{\zeta}}|\nabla \varphi|=c^{*}
$$

for some constant $\zeta>0$, where $\Omega_{\zeta}=\{(x, y) \in \Omega: \varphi(x, y)<\zeta\}$ and $\Gamma_{\zeta}=\overline{\partial \Omega_{\zeta} \cap \Omega}$. 


\subsection{Global smooth supersonic flows without vacuum in straight nozzles}

According to Theorems 3.2 and 2.3, under the assumptions of Theorem [3.2, the supersonic flow problem (2.31) - 2.35) admits a smooth solution before vacuum formation, furthermore, if in addition, the nozzle is straight, then the smooth supersonic flow is away from vacuum in any bounded region. Therefore, under the assumptions of Theorem 3.2 , there always exists a global smooth supersonic flow without sonic and vacuum point in a straight nozzle.

Theorem 3.3 Assume that $f \in C^{2}\left(\left[l_{0},+\infty\right)\right)$ satisfies (2.73), $\Upsilon \in C^{2}\left(\left[0, f\left(l_{0}\right)\right]\right)$ satisfies (2.36) and $q_{0} \in C^{1}\left(\left[0, f\left(l_{0}\right)\right]\right)$ satisfies (2.37)-(2.39). Then the problem (2.31) -(2.35) admits uniquely a smooth supersonic flow, which is away from vacuum in any bounded region. Furthermore, the solution $\varphi \in$ $C^{2}(\bar{\Omega})$ and $c_{1} \leq|\nabla \varphi|<c^{*}$ on $\bar{\Omega}$.

Below we indicate how to solve the Cauchy problem (2.60)-(2.62).

Theorem 3.4 Assume that $Q_{0} \in C^{1}(\mathbb{R})$ and $G_{0} \in C(\mathbb{R})$ satisfy

$$
-\mu_{2} \leq Q_{0}(\psi) \leq-\mu_{1}, \quad 0 \leq-G_{0}(\psi) \pm b^{1 / 2}\left(Q_{0}(\psi)\right) Q_{0}^{\prime}(\psi) \leq \mu_{3}, \quad \psi \in \mathbb{R}
$$

with three constants $0<\mu_{1} \leq \mu_{2}$ and $\mu_{3}>0$. Then the Cauchy problem (2.60)-(2.62) admits uniquely a solution $Q \in C^{1}([0,+\infty) \times \mathbb{R})$. Furthermore, the solution satisfies

$$
Q(\varphi, \psi) \leq-\mu_{1}, \quad 0 \leq-Q_{\varphi}(\varphi, \psi) \pm b^{1 / 2}(Q(\varphi, \psi)) Q_{\varphi}(\varphi, \psi) \leq \mu_{3}, \quad(\varphi, \psi) \in(0,+\infty) \times \mathbb{R} .
$$

Proof. The problem (2.60)-(2.62) is equivalent to

$$
\begin{array}{ll}
W_{\varphi}+b^{1 / 2}(Q) W_{\psi}=\frac{1}{4} b^{-1}(Q) p(Q) W(W+Z), & (\varphi, \psi) \in(0,+\infty) \times \mathbb{R}, \\
Z_{\varphi}-b^{1 / 2}(Q) Z_{\psi}=-\frac{1}{4} b^{-1}(Q) p(Q) Z(W+Z), & (\varphi, \psi) \in(0,+\infty) \times \mathbb{R}, \\
W(0, \psi)=W_{0}(\psi)=G_{0}(\psi)-b^{1 / 2}\left(Q_{0}(\psi)\right) Q_{0}^{\prime}(\psi), & \psi \in \mathbb{R}, \\
Z(0, \psi)=Z_{0}(\psi)=-G_{0}(\psi)-b^{1 / 2}\left(Q_{0}(\psi)\right) Q_{0}^{\prime}(\psi), & \psi \in \mathbb{R}, \\
Q_{\varphi}=\frac{1}{2}(W-Z), & (\varphi, \psi) \in(0,+\infty) \times \mathbb{R}, \\
Q(0, \psi)=Q_{0}(\psi), & \psi \in \mathbb{R} .
\end{array}
$$

Similar to the proof of Theorem 3.1, one can prove that the problem (2.60)-(2.62) admits uniquely a solution $Q \in C^{1}([0, \zeta) \times \mathbb{R})$, where either $\zeta=+\infty$ or $0<\zeta<+\infty$ with $\lim _{\varphi \rightarrow \zeta^{-}} \inf _{\mathbb{R}} Q(\varphi, \cdot)=-\infty$. Furthermore, the solution satisfies

$$
-Q_{\varphi}(\varphi, \psi) \pm b^{1 / 2}(Q(\varphi, \psi)) Q_{\psi}(\varphi, \psi) \geq 0, \quad(\varphi, \psi) \in(0, \zeta) \times \mathbb{R} .
$$

Proposition 2.1 shows that

$$
-Q_{\varphi}(\varphi, \psi) \pm b^{1 / 2}(Q(\varphi, \psi)) Q_{\psi}(\varphi, \psi) \leq \mu_{3}, \quad(\varphi, \psi) \in(0, \zeta) \times \mathbb{R} .
$$

Therefore, $\zeta=+\infty$ and $Q \in C^{1}([0,+\infty) \times \mathbb{R})$ satisfies (3.65). By the way, one can use another iteration scheme for the existence, where the cut-off technique is not needed. Fix $\tilde{\zeta}>0$ and define

$$
\mathscr{B}=\left\{Q \in C^{0,1}([0, \tilde{\zeta}] \times \mathbb{R}):-\mu_{2} \leq Q(0, \psi) \leq-\mu_{1} \text { for } \psi \in \mathbb{R},-\mu_{3} \leq Q_{\varphi}(\varphi, \psi) \leq 0\right.
$$




$$
\text { and } \left.\left|Q_{\psi}(\varphi, \psi)\right| \leq \beta \text { for }(\varphi, \psi) \in(0, \tilde{\zeta}) \times \mathbb{R}\right\}
$$

where $\beta>0$ is a constant to be determined. For fixed $\tilde{Q} \in \mathscr{B}$, consider the problem

$$
\begin{array}{ll}
W_{\varphi}+b^{1 / 2}(\tilde{Q}) W_{\psi}=\frac{1}{4} b^{-1}(\tilde{Q}) p(\tilde{Q}) W(W+Z), & (\varphi, \psi) \in(0, \tilde{\zeta}) \times \mathbb{R}, \\
Z_{\varphi}-b^{1 / 2}(\tilde{Q}) Z_{\psi}=-\frac{1}{4} b^{-1}(\tilde{Q}) p(\tilde{Q}) Z(W+Z), & (\varphi, \psi) \in(0, \tilde{\zeta}) \times \mathbb{R}, \\
W(0, \psi)=W_{0}(\psi), & \psi \in \mathbb{R}, \\
Z(0, \psi)=Z_{0}(\psi), & \psi \in \mathbb{R}, \\
Q_{\varphi}=\frac{1}{2}(W-Z), & (\varphi, \psi) \in(0, \tilde{\zeta}) \times \mathbb{R}, \\
Q(0, \psi)=Q_{0}(\psi), & \psi \in \mathbb{R} .
\end{array}
$$

The key estimate, which can be proved by the method of characteristics, is that for each $(w, z) \in$ $L^{\infty}((0, \tilde{\zeta}) \times \mathbb{R}) \times L^{\infty}((0, \tilde{\zeta}) \times \mathbb{R})$ with

$$
-\mu_{3} \leq w(\varphi, \psi) \leq 0, \quad 0 \leq z(\varphi, \psi) \leq \mu_{3}, \quad(\varphi, \psi) \in(0, \tilde{\zeta}) \times \mathbb{R}
$$

the problem

$$
\begin{array}{ll}
W_{\varphi}+b^{1 / 2}(\tilde{Q}) W_{\psi}=\frac{1}{4} b^{-1}(\tilde{Q}) p(\tilde{Q}) W(W+z), & (\varphi, \psi) \in(0, \tilde{\zeta}) \times \mathbb{R}, \\
Z_{\varphi}-b^{1 / 2}(\tilde{Q}) Z_{\psi}=-\frac{1}{4} b^{-1}(\tilde{Q}) p(\tilde{Q}) Z(w+Z), & (\varphi, \psi) \in(0, \tilde{\zeta}) \times \mathbb{R}, \\
W(0, \psi)=W_{0}(\psi), & \psi \in \mathbb{R}, \\
Z(0, \psi)=Z_{0}(\psi), & \psi \in \mathbb{R}
\end{array}
$$

admits uniquely a weak solution $(W, Z) \in L^{\infty}((0, \tilde{\zeta}) \times \mathbb{R}) \times L^{\infty}((0, \tilde{\zeta}) \times \mathbb{R})$, and the solution satisfies

$$
-\mu_{3} \leq W(\varphi, \psi) \leq 0, \quad 0 \leq Z(\varphi, \psi) \leq \mu_{3}, \quad(\varphi, \psi) \in(0, \tilde{\zeta}) \times \mathbb{R} .
$$

Based on this key estimate, the problem (3.66)-3.69) admits a unique weak solution $(W, Z) \in$ $L^{\infty}((0, \tilde{\zeta}) \times \mathbb{R}) \times L^{\infty}((0, \tilde{\zeta}) \times \mathbb{R})$ satisfying

$$
-\mu_{3} \leq W(\varphi, \psi) \leq 0, \quad 0 \leq Z(\varphi, \psi) \leq \mu_{3}, \quad(\varphi, \psi) \in(0, \tilde{\zeta}) \times \mathbb{R} .
$$

Then, similar to the proof of Lemma 3.2, one can show that the problem (3.70), (3.71) admits a unique weak solution $Q \in C^{0,1}([0, \tilde{\zeta}] \times \mathbb{R})$ and it satisfies

$$
-\mu_{3} \leq Q_{\varphi}(\varphi, \psi) \leq 0, \quad\left|Q_{\psi}(\varphi, \psi)\right| \leq \beta, \quad(\varphi, \psi) \in(0, \tilde{\zeta}) \times \mathbb{R}
$$

with a positive constant $\beta=\beta\left(\gamma, \mu_{1}, \mu_{2}, \mu_{3}, \tilde{\zeta}\right)$.

Remark 3.5 The key estimate in Theorem 3.4 is invalid for the problem (2.40)-(2.45) with a nonhomogeneous boundary condition at $\psi=m$. So, the new iteration scheme in Theorem 3.4 is unsuitable for Theorem 3.1. 


\subsection{Properties of smooth supersonic flows before vacuum formation}

In this subsection, we investigate the properties of smooth supersonic flows before vacuum formation, including the lower bound estimate of the flow speed in the potential plane and the Lipschitz continuity of the flow speed in the physical plane.

Proposition 3.1 Let $f \in C^{2}\left(\left[l_{0}, l_{1}\right)\right)$ satisfy (2.30), and $Q_{0} \in C^{1}([0, m])$ and $G_{0} \in C([0, m])$ satisfy (2.47), (2.48) and

$$
-G_{0}(\psi) \pm b^{1 / 2}\left(Q_{0}(\psi)\right) Q_{0}^{\prime}(\psi) \geq \mu_{0}, \quad \psi \in(0, m)
$$

where $\mu_{0}>0$ is a positive constant. Assume that $Q \in C^{1}([0, \zeta) \times[0, m])$ is a solution to the problem (2.40) - (2.45) with some $0<\zeta \leq+\infty$. Then there exists a positive constant $M=M\left(\gamma, m, \mu_{0}, \mu_{1}\right)$ such that

$$
Q(\varphi, \psi) \leq-M(\varphi+1)^{2 /(\gamma+1)}, \quad(\varphi, \psi) \in(0, \zeta) \times(0, m)
$$

If, in addition, $f^{\prime \prime}\left(1+\left(f^{\prime}\right)^{2}\right)^{-3 / 2} \in L^{\infty}\left(l_{0}, l_{1}\right)$, then

$$
-N \leq b^{1 / 2}(Q(\varphi, \psi)) Q_{\varphi}(\varphi, \psi) \leq 0, \quad\left|b(Q(\varphi, \psi)) Q_{\psi}(\varphi, \psi)\right| \leq N, \quad(\varphi, \psi) \in(0, \zeta) \times(0, m),
$$

where $N=N\left(\gamma, m, \mu_{0}, \mu_{1}, \mu_{3},\left\|f^{\prime \prime}\left(1+\left(f^{\prime}\right)^{2}\right)^{-3 / 2}\right\|_{L^{\infty}\left(l_{0}, l_{1}\right)}\right)$ is a positive constant.

Proof. For $(\varphi, \psi) \in[0, \zeta) \times[0, m]$, set

$$
W(\varphi, \psi)=Q_{\varphi}(\varphi, \psi)-b^{1 / 2}(Q(\varphi, \psi)) Q_{\psi}(\varphi, \psi), \quad Z(\varphi, \psi)=-Q_{\varphi}(\varphi, \psi)-b^{1 / 2}(Q(\varphi, \psi)) Q_{\psi}(\varphi, \psi) .
$$

Theorem 3.1 shows that

$$
Q(\varphi, \psi) \leq-\mu_{1}, \quad(\varphi, \psi) \in(0, \zeta) \times(0, m)
$$

and $(W, Z) \in C([0, \zeta) \times[0, m]) \times C([0, \zeta) \times[0, m])$ solves

$$
\begin{array}{ll}
W_{\varphi}+b^{1 / 2}(Q) W_{\psi}=\frac{1}{4} b^{-1}(Q) p(Q) W(W+Z), & (\varphi, \psi) \in(0, \zeta) \times(0, m), \\
Z_{\varphi}-b^{1 / 2}(Q) Z_{\psi}=-\frac{1}{4} b^{-1}(Q) p(Q) Z(W+Z), & (\varphi, \psi) \in(0, \zeta) \times(0, m), \\
W(0, \psi)=W_{0}(\psi)=G_{0}(\psi)-b^{1 / 2}\left(Q_{0}(\psi)\right) Q_{0}^{\prime}(\psi) \leq-\mu_{0}, & \psi \in(0, m), \\
Z(0, \psi)=Z_{0}(\psi)=-G_{0}(\psi)-b^{1 / 2}\left(Q_{0}(\psi)\right) Q_{0}^{\prime}(\psi) \geq \mu_{0}, & \psi \in(0, m), \\
W(\varphi, 0)+Z(\varphi, 0)=0, & \varphi \in(0, \zeta), \\
W(\varphi, m)+Z(\varphi, m)=\frac{2 f^{\prime \prime}\left(X_{\mathrm{up}}(\varphi)\right)\left(1+\left(f^{\prime}\left(X_{\mathrm{up}}(\varphi)\right)\right)^{2}\right)^{-3 / 2}}{b^{1 / 2}(Q(\varphi, m)) A_{+}^{-1}(Q(\varphi, m))} \geq 0, & \varphi \in(0, \zeta) .
\end{array}
$$

By the method of characteristics, it is clear that

$$
W(\varphi, \psi)<0, \quad Z(\varphi, \psi)>0, \quad(\varphi, \psi) \in(0, \zeta) \times(0, m) .
$$

Assume that

$$
\Sigma_{ \pm}: \Psi_{ \pm}^{\prime}(\varphi)= \pm b^{1 / 2}\left(Q\left(\varphi, \Psi_{ \pm}(\varphi)\right)\right), \quad 0<\Psi_{ \pm}(\varphi)<m, \quad \hat{\varphi}_{ \pm}<\varphi<\check{\varphi}_{ \pm} \quad\left(0 \leq \hat{\varphi}_{ \pm}<\check{\varphi}_{ \pm}<\zeta\right)
$$


are a positive and a negative characteristics. It follows from (3.75) and (3.80) that $W$ and $Z$ satisfy

$$
\frac{d}{d \varphi} W\left(\varphi, \Psi_{+}(\varphi)\right) \leq-\frac{M_{1}}{Q\left(\varphi, \Psi_{+}(\varphi)\right)} W^{2}\left(\varphi, \Psi_{+}(\varphi)\right), \quad \hat{\varphi}_{+}<\varphi<\check{\varphi}_{+}
$$

and

$$
\frac{d}{d \varphi} Z\left(\varphi, \Psi_{-}(\varphi)\right) \geq \frac{M_{1}}{Q\left(\varphi, \Psi_{+}(\varphi)\right)} Z^{2}\left(\varphi, \Psi_{-}(\varphi)\right), \quad \hat{\varphi}_{-}<\varphi<\check{\varphi}_{-}
$$

on $\Sigma_{ \pm}$, respectively, where $M_{1}=M_{1}\left(\gamma, \mu_{1}\right)$ is a positive constant. Therefore,

$$
\begin{array}{ll}
\frac{d}{d \varphi}\left(\frac{1}{W\left(\varphi, \Psi_{+}(\varphi)\right)}\right) \geq \frac{M_{1}}{Q\left(\varphi, \Psi_{+}(\varphi)\right)}, & \hat{\varphi}_{+}<\varphi<\check{\varphi}_{+}, \\
\frac{d}{d \varphi}\left(\frac{1}{Z\left(\varphi, \Psi_{-}(\varphi)\right)}\right) \leq-\frac{M_{1}}{Q\left(\varphi, \Psi_{-}(\varphi)\right)}, & \hat{\varphi}_{-}<\varphi<\check{\varphi}_{-}
\end{array}
$$

which, together with (3.75), yield

$$
\begin{array}{ll}
\frac{d}{d \varphi}\left(\frac{1}{W\left(\varphi, \Psi_{+}(\varphi)\right)}\right) \geq-\frac{M_{1}}{\mu_{1}}, & \hat{\varphi}_{+}<\varphi<\check{\varphi}_{+}, \\
\frac{d}{d \varphi}\left(\frac{1}{Z\left(\varphi, \Psi_{-}(\varphi)\right)}\right) \leq \frac{M_{1}}{\mu_{1}}, & \hat{\varphi}_{-}<\varphi<\check{\varphi}_{-} .
\end{array}
$$

Direct calculation shows

$$
\begin{aligned}
& \frac{d}{d \varphi}\left(\frac{1}{\left(-Q\left(\varphi, \Psi_{+}(\varphi)\right)\right)^{(\gamma-1) / 2} W\left(\varphi, \Psi_{+}(\varphi)\right)}\right) \\
& =\frac{1}{\left(-Q\left(\varphi, \Psi_{+}(\varphi)\right)\right)^{(\gamma-1) / 2}} \frac{d}{d \varphi}\left(\frac{1}{W\left(\varphi, \Psi_{+}(\varphi)\right)}\right) \\
& +\frac{\gamma-1}{2} \frac{Q_{\varphi}\left(\varphi, \Psi_{+}(\varphi)\right)+b^{1 / 2}(\varphi) Q_{\psi}\left(\varphi, \Psi_{+}(\varphi)\right)}{\left(-Q\left(\varphi, \Psi_{+}(\varphi)\right)\right)^{(\gamma-1) / 2+1} W\left(\varphi, \Psi_{+}(\varphi)\right)} \\
& =\frac{1}{\left(-Q\left(\varphi, \Psi_{+}(\varphi)\right)\right)^{(\gamma-1) / 2}} \frac{d}{d \varphi}\left(\frac{1}{W\left(\varphi, \Psi_{+}(\varphi)\right)}\right) \\
& -\frac{\gamma-1}{2} \frac{Z\left(\varphi, \Psi_{+}(\varphi)\right)}{\left(-Q\left(\varphi, \Psi_{+}(\varphi)\right)\right)^{(\gamma-1) / 2+1} W\left(\varphi, \Psi_{+}(\varphi)\right)}, \quad \hat{\varphi}_{+}<\varphi<\check{\varphi}_{+},
\end{aligned}
$$

which, together with (3.80), (3.81) and (3.75), leads to

$$
\begin{aligned}
\frac{d}{d \varphi}\left(\frac{1}{\left(-Q\left(\varphi, \Psi_{+}(\varphi)\right)\right)^{(\gamma-1) / 2} W\left(\varphi, \Psi_{+}(\varphi)\right)}\right) & \geq-\frac{M_{1}}{\left(-Q\left(\varphi, \Psi_{+}(\varphi)\right)\right)^{(\gamma+1) / 2}} \\
& \geq-M_{2} b^{1 / 2}\left(Q\left(\varphi, \Psi_{+}(\varphi)\right)\right), \quad \hat{\varphi}_{+}<\varphi<\check{\varphi}_{+},
\end{aligned}
$$

where $M_{2}=M_{2}\left(\gamma, \mu_{1}\right)$ is a positive constant. Similarly, one gets from (3.80), (3.82) and (3.75) that

$$
\frac{d}{d \varphi}\left(\frac{1}{\left(-Q\left(\varphi, \Psi_{-}(\varphi)\right)\right)^{(\gamma-1) / 2} Z\left(\varphi, \Psi_{-}(\varphi)\right)}\right) \leq M_{2} b^{1 / 2}\left(Q\left(\varphi, \Psi_{-}(\varphi)\right)\right), \quad \hat{\varphi}_{-}<\varphi<\check{\varphi}_{-} .
$$

Fix $\left(\varphi_{0}, \psi_{0}\right) \in(0, \zeta) \times(0, m)$. Similar to the proof of Lemma 3.1, there exists two nonnegative integers $k_{ \pm}$such that

$$
\varphi_{0}=\varphi_{0}^{ \pm}>\varphi_{1}^{ \pm}>\cdots>\varphi_{k_{ \pm}}^{ \pm}>\varphi_{k_{ \pm}+1}^{ \pm}=0, \quad \psi_{0}^{ \pm}=\psi_{0}, \quad 0 \leq \psi_{k_{ \pm}+1}^{ \pm} \leq m
$$




$$
\begin{aligned}
& \psi_{j}^{+}=\left\{\begin{array}{l}
0, \quad 1 \leq j \leq k_{+} \text {and } j \text { is odd, } \\
m, \quad 1 \leq j \leq k_{+} \text {and } j \text { is even, }
\end{array} \psi_{j}^{-}=\left\{\begin{array}{l}
m, \quad 1 \leq j \leq k_{-} \text {and } j \text { is odd, } \\
0, \quad 1 \leq j \leq k_{-} \text {and } j \text { is even, }
\end{array}\right.\right. \\
& \left\{\begin{array}{l}
\frac{d}{d \varphi} \Psi_{j}^{ \pm}(\varphi)= \pm(-1)^{j-1} b^{1 / 2}\left(Q\left(\varphi, \Psi_{j}^{ \pm}(\varphi)\right)\right), \quad \varphi_{j}^{ \pm}<\varphi<\varphi_{j-1}^{ \pm}, \quad 1 \leq j \leq k_{ \pm}+1 . \\
\Psi_{j}^{ \pm}\left(\varphi_{j}^{ \pm}\right)=\psi_{j}^{ \pm}, \quad \Psi_{j}^{ \pm}\left(\varphi_{j-1}^{ \pm}\right)=\psi_{j-1}^{ \pm},
\end{array}\right.
\end{aligned}
$$

Below we estimate $W\left(\varphi_{0}, \psi_{0}\right)$ and $Z\left(\varphi_{0}, \psi_{0}\right)$ by three steps.

Step I Rough estimates for $W\left(\varphi_{0}, \psi_{0}\right)$ and $Z\left(\varphi_{0}, \psi_{0}\right)$.

For $1 \leq j \leq k_{+}+1$, (3.83) and (3.84) show

$$
\begin{array}{ll}
\frac{1}{W\left(\varphi_{j-1}^{+}, \psi_{j-1}^{+}\right)} \geq \frac{1}{W\left(\varphi_{j}^{+}, \psi_{j}^{+}\right)}-\frac{M_{1}}{\mu_{1}}\left(\varphi_{j-1}^{+}-\varphi_{j}^{+}\right), & \text {if } j \text { is odd, } \\
\frac{1}{Z\left(\varphi_{j-1}^{+}, \psi_{j-1}^{+}\right)} \leq \frac{1}{Z\left(\varphi_{j}^{+}, \psi_{j}^{+}\right)}+\frac{M_{1}}{\mu_{1}}\left(\varphi_{j-1}^{+}-\varphi_{j}^{+}\right), & \text {if } j \text { is even, }
\end{array}
$$

which, together with (3.76) (3.79), lead to

$$
\frac{1}{W\left(\varphi_{0}, \psi_{0}\right)} \geq-\frac{1}{\mu_{0}}-\frac{M_{1}}{\mu_{1}} \varphi_{0} .
$$

Similarly, one can show that

$$
\frac{1}{Z\left(\varphi_{0}, \psi_{0}\right)} \leq \frac{1}{\mu_{0}}+\frac{M_{1}}{\mu_{1}} \varphi_{0}
$$

Step II Upper bounds for $k_{ \pm}$.

By (3.87), (3.88) and the arbitrariness of $\left(\varphi_{0}, \psi_{0}\right) \in(0, \zeta) \times(0, m)$, one gets that

$$
Q_{\varphi}(\varphi, \psi) \leq-\frac{1}{1 / \mu_{0}+M_{1} \varphi / \mu_{1}}, \quad(\varphi, \psi) \in(0, \zeta) \times(0, m)
$$

which yields

$$
Q(\varphi, \psi) \leq-M_{3}(1+\ln (\varphi+1)), \quad(\varphi, \psi) \in(0, \zeta) \times(0, m)
$$

where $M_{3}=M_{3}\left(\gamma, \mu_{0}, \mu_{1}\right)$ is a positive constant. It follows from (3.72) that $\theta_{\psi}(0, \cdot) \geq \mu_{0}$ in $(0, m)$, which, together with $\theta(0,0)=0$, leads to $f^{\prime}\left(l_{0}\right)=\tan \theta(0, m) \geq \tan \left(\mu_{0} m\right)$. Choose a positive constant $M_{4}=M_{4}\left(\gamma, m, \mu_{0}, \mu_{1}\right) \geq 0$ such that

$$
\mathscr{H}\left(-M_{3}\left(1+\ln \left(M_{4}+1\right)\right)\right)>\mathscr{H}(-\infty)-\arctan f^{\prime}\left(l_{0}\right) .
$$

Note that (2.30) yields

$$
\theta_{\varphi}(\varphi, m)=\frac{d}{d \varphi} \arctan f^{\prime}\left(X_{\mathrm{up}}(\varphi)\right)=\frac{f^{\prime \prime}\left(X_{\mathrm{up}}(\varphi) X_{\mathrm{up}}^{\prime}(\varphi)\right.}{1+\left(f^{\prime}\left(X_{\mathrm{up}}(\varphi)\right)^{2}\right.} \geq 0, \quad 0 \leq \varphi<\zeta .
$$

If $0<\zeta \leq M_{4}$, then (3.75) yields $k_{ \pm} \leq b^{1 / 2}\left(-\mu_{1}\right) M_{4} / m+1$. In the other case that $M_{4}<\zeta \leq+\infty$, for each $\tilde{\varphi} \geq M_{4}$, Lemma 2.1, (3.89) (3.91) show that the positive characteristic from $(\tilde{\varphi}, 0)$ must not touch $(0, \zeta) \times\{m\}$ and the negative characteristic from $(\tilde{\varphi}, m)$ must not touch $(0, \zeta) \times\{0\}$. Therefore, there exists a positive integer $K=K\left(\gamma, m, \mu_{0}, \mu_{1}\right)$ such that in both cases,

$$
k_{ \pm} \leq K
$$




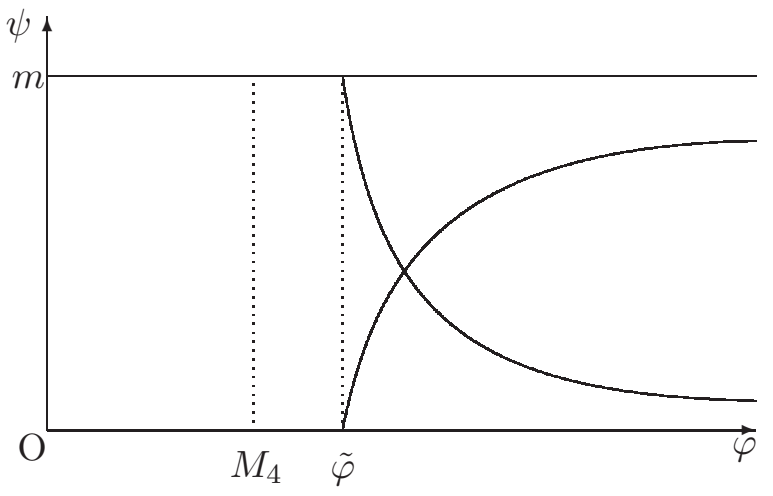

Step III Refined estimates for $W\left(\varphi_{0}, \psi_{0}\right)$ and $Z\left(\varphi_{0}, \psi_{0}\right)$.

For $1 \leq j \leq k_{+}+1$, (3.85) and (3.86) show

$\begin{array}{ll}\frac{1}{\left(-Q\left(\varphi_{j-1}^{+}, \psi_{j-1}^{+}\right)\right)^{(\gamma-1) / 2} W\left(\varphi_{j-1}^{+}, \psi_{j-1}^{+}\right)} \geq \frac{1}{\left(-Q\left(\varphi_{j}^{+}, \psi_{j}^{+}\right)\right)^{(\gamma-1) / 2} W\left(\varphi_{j}^{+}, \psi_{j}^{+}\right)}-M_{2} m, & \text { if } j \text { is odd, } \\ \frac{1}{\left(-Q\left(\varphi_{j-1}^{+}, \psi_{j-1}^{+}\right)\right)^{(\gamma-1) / 2} Z\left(\varphi_{j-1}^{+}, \psi_{j-1}^{+}\right)} \leq \frac{1}{\left(-Q\left(\varphi_{j}^{+}, \psi_{j}^{+}\right)\right)^{(\gamma-1) / 2} Z\left(\varphi_{j}^{+}, \psi_{j}^{+}\right)}+M_{2} m, & \text { if } j \text { is even, }\end{array}$

which, together with (3.76)- (3.79) and (3.92), yield

$$
\frac{1}{\left(-Q\left(\varphi_{0}, \psi_{0}\right)\right)^{(\gamma-1) / 2} W\left(\varphi_{0}, \psi_{0}\right)} \geq-\frac{1}{\mu_{1}^{(\gamma-1) / 2} \mu_{0}}-(K+1) M_{2} m .
$$

Similarly, one can show that

$$
\frac{1}{\left(-Q\left(\varphi_{0}, \psi_{0}\right)\right)^{(\gamma-1) / 2} Z\left(\varphi_{0}, \psi_{0}\right)} \leq \frac{1}{\mu_{1}^{(\gamma-1) / 2} \mu_{0}}+(K+1) M_{2} m .
$$

It follows from (3.93), (3.94) and the arbitrariness of $\left(\varphi_{0}, \psi_{0}\right) \in(0, \zeta) \times(0, m)$ that

$$
(-Q(\varphi, \psi))^{(\gamma-1) / 2} Q_{\varphi}(\varphi, \psi) \leq-\frac{1}{1 /\left(\mu_{1}^{(\gamma-1) / 2} \mu_{0}\right)+(K+1) M_{2} m}, \quad(\varphi, \psi) \in(0, \zeta) \times(0, m),
$$

which yields (3.73) directly.

Below we prove (3.74). Set

$$
\begin{array}{lll}
\mathscr{U}(\varphi, \psi)=b^{1 / 2}(Q(\varphi, \psi)) Q_{\varphi}(\varphi, \psi)-b(Q(\varphi, \psi)) Q_{\psi}(\varphi, \psi), & (\varphi, \psi) \in[0, \zeta) \times[0, m], \\
\mathscr{V}(\varphi, \psi)=-b^{1 / 2}(Q(\varphi, \psi)) Q_{\varphi}(\varphi, \psi)-b(Q(\varphi, \psi)) Q_{\psi}(\varphi, \psi), & (\varphi, \psi) \in[0, \zeta) \times[0, m] .
\end{array}
$$

Theorem 3.1 shows

$$
\mathscr{U}(\varphi, \psi) \leq 0, \quad \mathscr{V}(\varphi, \psi) \geq 0, \quad(\varphi, \psi) \in(0, \zeta) \times(0, m)
$$

It follows from (2.49)-(2.57) and (3.95) that $(\mathscr{U}, \mathscr{V}) \in C([0, \zeta) \times[0, m]) \times C([0, \zeta) \times[0, m])$ solves

$$
\begin{array}{ll}
\mathscr{U}_{\varphi}+b^{1 / 2}(Q) \mathscr{U}_{\psi}=\frac{1}{4} b^{-3 / 2}(Q) p(Q) \mathscr{U}(\mathscr{U}-\mathscr{V}) \geq 0, & (\varphi, \psi) \in(0, \zeta) \times(0, m), \\
\mathscr{V}_{\varphi}-b^{1 / 2}(Q) \mathscr{V}_{\psi}=\frac{1}{4} b^{-3 / 2}(Q) p(Q) \mathscr{V}(\mathscr{U}-\mathscr{V}) \leq 0, & (\varphi, \psi) \in(0, \zeta) \times(0, m), \\
\mathscr{U}(\varphi, 0)+\mathscr{V}(\varphi, 0)=0, & \varphi \in(0, \zeta),
\end{array}
$$




$$
\mathscr{U}(\varphi, m)+\mathscr{V}(\varphi, m)=\frac{2 f^{\prime \prime}\left(X_{\mathrm{up}}(\varphi)\right)\left(1+\left(f^{\prime}\left(X_{\mathrm{up}}(\varphi)\right)\right)^{2}\right)^{-3 / 2}}{A_{+}^{-1}(Q(\varphi, m))}, \quad \varphi \in(0, \zeta)
$$

By $f^{\prime \prime}\left(1+\left(f^{\prime}\right)^{2}\right)^{-3 / 2} \in L^{\infty}\left(l_{0}, l_{1}\right)$ and (3.92) , one can prove by the method of characteristics that

$$
-N \leq \mathscr{U}(\varphi, \psi) \leq 0, \quad 0 \leq \mathscr{V}(\varphi, \psi) \leq N, \quad(\varphi, \psi) \in(0, \zeta) \times(0, m)
$$

with $N=N\left(\gamma, m, \mu_{0}, \mu_{1}, \mu_{3},\left\|f^{\prime \prime}\left(1+\left(f^{\prime}\right)^{2}\right)^{-3 / 2}\right\|_{L^{\infty}\left(l_{0}, l_{1}\right)}\right)$ being a positive constant, which yields (3.74) directly.

Remark 3.6 If $q_{0}$ satisfies

$$
\inf _{\left(0, f\left(l_{+}\right)\right)}\left(\frac{-\Upsilon^{\prime \prime}}{1+\left(\Upsilon^{\prime}\right)^{2}} \sqrt{\frac{-q_{0}^{2} \rho\left(q_{0}^{2}\right)}{\rho\left(q_{0}^{2}\right)+2 q_{0}^{2} \rho^{\prime}\left(q_{0}^{2}\right)}}-\left|q_{0}^{\prime}\right|\right)>0
$$

then (3.72) holds.

It can be verified easily that

Remark 3.7 For the case $0<\zeta<+\infty$, (3.74) remains true even if $f^{\prime \prime}\left(1+\left(f^{\prime}\right)^{2}\right)^{-3 / 2} \notin L^{\infty}\left(l_{0}, l_{1}\right)$ and (3.72) is not satisfied. Here, the constant $N$ depends only on $\gamma, m, \mu_{1}, \mu_{3}, \zeta$ and $f$.

As an application of Proposition 3.1 in the physical plane, one gets

Theorem 3.5 Assume that $f \in C^{2}\left(\left[l_{0}, l_{1}\right)\right)$ satisfies (2.30) and $f^{\prime \prime}\left(1+\left(f^{\prime}\right)^{2}\right)^{-3 / 2} \in L^{\infty}\left(l_{0}, l_{1}\right), \Upsilon \in$ $C^{2}\left(\left[0, f\left(l_{0}\right)\right]\right)$ satisfies (2.36) and $q_{0} \in C^{1}\left(\left[0, f\left(l_{0}\right)\right]\right)$ satisfies (2.37), (3.96) and (2.39). Let $\varphi \in$ $C^{2}(\bar{\Omega})$ be a global supersonic flow without vacuum to the problem (2.31)-(2.35). Then $|\nabla \varphi|$ is globally Lipschitz continuous in $\Omega$.

Remark 3.8 As will be shown in Theorem 4.4, if $\frac{\lim }{x \rightarrow l_{1}^{-}} f^{\prime \prime}(x)\left(1+\left(f^{\prime}(x)\right)^{2}\right)^{-3 / 2}>0$, the smooth supersonic flow to the problem (2.31) -(2.35) must admit a vacuum.

\section{Formation of vacuum in a smooth supersonic flow}

For a smooth supersonic flow with vacuum to the problem (2.31)-(2.35), it is clear that the set of vacuum points is closed and thus there exists the first vacuum point in the increasing $x$-direction. In this section, we will study the formation of the first vacuum point.

\subsection{Location of the first vacuum point and behavior of the flow near this point}

The following theorem shows that the first vacuum point of the flow in the increasing $\varphi$-direction must form at the upper wall.

Theorem 4.1 Assume that $f \in C^{2}\left(\left[l_{0}, l_{1}\right)\right)$ satisfies (2.30), and $Q_{0} \in C^{1}([0, m])$ and $G_{0} \in C([0, m])$ satisfy (2.47) and (2.48). Let $Q \in C^{1}([0, \zeta) \times[0, m])$ with $0<\zeta<+\infty$ be the maximal smooth supersonic flow before vacuum formation to the problem (2.40)-(2.45). Then $q, \theta \in C([0, \zeta] \times[0, m]) \cap$ $C^{1}([0, \zeta] \times[0, m] \backslash\{(\zeta, m)\})$ satisfying

$$
|q(\hat{\varphi}, \hat{\psi})-q(\check{\varphi}, \check{\psi})| \leq M\left(|\hat{\varphi}-\check{\varphi}|+|\hat{\psi}-\check{\psi}|^{1-1 / \gamma}\right), \quad(\hat{\varphi}, \hat{\psi}),(\check{\varphi}, \check{\psi}) \in(0, \zeta) \times(0, m),
$$




$$
\begin{gathered}
q(\zeta, m)=c^{*}, \quad q(\varphi, \psi)<c^{*}, \quad(\varphi, \psi) \in[0, \zeta] \times[0, m] \backslash\{(\zeta, m)\} \\
q(\varphi, m) \geq c^{*}-M(\zeta-\varphi)^{2}, \quad 0<\varphi<\zeta \\
\left|\theta_{\varphi}(\varphi, \psi)\right| \leq M, \quad 0 \leq\left(c^{*}-q(\varphi, \psi)\right)^{1 /(\gamma-1)+1 / 2} \theta_{\psi}(\varphi, \psi) \leq M, \quad(\varphi, \psi) \in[0, \zeta] \times[0, m] \backslash\{(\zeta, m)\},
\end{gathered}
$$

where $M=M\left(\gamma, m, \mu_{1}, \mu_{3}, \zeta, f\right)$ is a positive constant.

Proof. It follows from Proposition 3.1 and Remark 3.7 that

$$
-M_{1} \leq \mathscr{U}(\varphi, \psi) \leq 0, \quad 0 \leq \mathscr{V}(\varphi, \psi) \leq M_{1}, \quad(\varphi, \psi) \in(0, \zeta) \times(0, m)
$$

with $M_{1}=M_{1}\left(\gamma, m, \mu_{1}, \mu_{3}, \zeta, f\right)$ being a positive constant. Theorem 3.1 shows $c_{*}<A_{+}^{-1}\left(-\mu_{1}\right) \leq q<$ $c^{*}$ in $(0, \zeta) \times(0, m)$, which, together with (4.5), yields that for each $(\varphi, \psi) \in(0, \zeta) \times(0, m)$,

$$
\begin{gathered}
0 \leq\left(c^{*}-q(\varphi, \psi)\right)^{-1 / 2} q_{\varphi}(\varphi, \psi) \leq M_{2}, \quad\left|\left(c^{*}-q(\varphi, \psi)\right)^{1 /(\gamma-1)} q_{\psi}(\varphi, \psi)\right| \leq M_{2}, \\
\left|\theta_{\varphi}(\varphi, \psi)\right| \leq M_{2}, \quad 0 \leq\left(c^{*}-q(\varphi, \psi)\right)^{1 /(\gamma-1)+1 / 2} \theta_{\psi}(\varphi, \psi) \leq M_{2},
\end{gathered}
$$

where $M_{2}=M_{2}\left(\gamma, m, \mu_{1}, \mu_{3}, \zeta, f\right)$ is a positive constant. It follows from (4.6) that $q \in C([0, \zeta] \times[0, m])$ and it satisfies (4.1). Theorem 3.1 yields $0<b^{1 / 2}(Q) \leq b^{1 / 2}\left(-\mu_{1}\right)$ in $(0, \zeta) \times(0, m)$. Therefore, for each $\max \left\{0, \zeta-m b^{-1 / 2}\left(-\mu_{1}\right)\right\}<\tilde{\varphi}<\zeta$, Lemma 2.2 shows that $Q \in C^{1}(\overline{\tilde{\omega}})$ with

$$
\tilde{\omega}=\left\{(\varphi, \psi) \in(\tilde{\varphi}, \zeta) \times(0, m): \psi<m-b^{1 / 2}\left(-\mu_{1}\right)(\varphi-\tilde{\varphi})\right\} .
$$

Hence $Q \in C^{1}([0, \zeta] \times[0, m] \backslash\{(\zeta, m)\})$, which, together with $q \in C([0, \zeta] \times[0, m])$ and $\lim _{\varphi \rightarrow \zeta^{-}} \min _{[0, m]} Q(\varphi, \cdot)=$ $-\infty$, leads to (4.2) and $q, \theta \in C^{1}([0, \zeta] \times[0, m] \backslash\{(\zeta, m)\})$. Finally, (4.3) follows from the first formula in (4.2) and the first estimate in (4.6), while $\theta \in C([0, \zeta] \times[0, m])$ and (4.4) follow from (4.7).

Below we characterize the boundary conditions of a local smooth supersonic flow at the potential level set where the first vacuum forms. Assume that $Q \in C^{1}([0, \zeta) \times[0, m])$ with $0<\zeta<+\infty$ is a maximal smooth supersonic flow before vacuum formation to the problem (2.40)-(2.45), which is $\varphi \in C^{2}\left(\bar{\Omega}_{\zeta} \backslash \Gamma_{\zeta}\right)$ to the problem (2.31) -(2.35) in the physical plane. Theorems 4.1 and 3.1 show that the first vacuum point of the flow in the increasing $x$-direction must form at the upper wall, which is $\left(X_{\text {up }}(\zeta), f\left(X_{\text {up }}(\zeta)\right)\right)$. For convenience, denote $x_{0}=X_{\text {up }}(\zeta)$ and $y_{0}=f\left(X_{\text {up }}(\zeta)\right)$. It follows from Theorems 3.1 and 4.1 that $Q \in C^{1}([0, \zeta] \times[0, m] \backslash\{(\zeta, m)\})$ satisfies

$$
-Q_{\varphi}(\varphi, \psi) \pm b^{1 / 2}(Q(\varphi, \psi)) Q_{\psi}(\varphi, \psi) \geq 0, \quad(\varphi, \psi) \in[0, \zeta] \times[0, m] \backslash\{(\zeta, m)\},
$$

$q, \theta \in C([0, \zeta] \times[0, m])$ satisfy $q(\zeta, m)=c^{*}$ and $\theta(\zeta, m)=\arctan f^{\prime}\left(x_{0}\right)>0$. Set

$$
\tilde{Q}_{0}(\psi)=Q(\zeta, \psi), \quad \tilde{G}_{0}(\psi)=Q_{\varphi}(\zeta, \psi), \quad \psi \in[0, m) .
$$

Then, $\tilde{Q}_{0} \in C^{1}([0, m))$ and $\tilde{G}_{0} \in C([0, m)) \cap L^{1}(0, m)$ satisfying

$$
\begin{gathered}
\tilde{Q}_{0}(\psi)<0, \quad-\tilde{G}_{0}(\psi) \pm b^{1 / 2}\left(\tilde{Q}_{0}(\psi)\right) \tilde{Q}_{0}^{\prime}(\psi) \geq 0, \quad \psi \in[0, m), \\
\tilde{Q}_{0}^{\prime}(0)=0, \quad \lim _{\psi \rightarrow m^{-}} \tilde{Q}_{0}(\psi)=-\infty, \quad \int_{0}^{m} \tilde{G}_{0}(\psi) d \psi=\theta(\zeta, m)=\arctan f^{\prime}\left(x_{0}\right) .
\end{gathered}
$$

In the physical plane, $\varphi \in C^{2}\left(\bar{\Omega}_{\zeta} \backslash\left\{\left(x_{0}, y_{0}\right)\right\}\right) \cap C^{1}\left(\bar{\Omega}_{\zeta}\right)$, and $\Gamma_{\zeta}$ is a graph of function with respect to $y$ on $\left[0, y_{0}\right]$, which is denoted by $\tilde{\Upsilon}$. Set

$$
\tilde{q}_{0}(y)=|\nabla \varphi(\tilde{\Upsilon}(y), y)|, \quad y \in\left[0, y_{0}\right]
$$


Then, $\tilde{\Upsilon} \in C^{2}\left(\left[0, y_{0}\right)\right) \cap C^{1}\left(\left[0, y_{0}\right]\right)$ and $\tilde{q}_{0} \in C^{1}\left(\left[0, y_{0}\right)\right) \cap C\left(\left[0, y_{0}\right]\right)$ satisfying

$$
\begin{gathered}
\tilde{\Upsilon}\left(y_{0}\right)=x_{0}, \quad \tilde{\Upsilon}^{\prime}(0)=0, \quad \tilde{\Upsilon}^{\prime}\left(y_{0}\right)=-f^{\prime}\left(x_{0}\right), \quad \tilde{q}_{0}^{\prime}(0)=0, \quad \tilde{q}_{0}\left(y_{0}\right)=c^{*}, \\
c_{*}<\tilde{q}_{0}(y)<c^{*}, \quad\left|\tilde{q}_{0}^{\prime}(y)\right| \leq \frac{-\tilde{\Upsilon}^{\prime \prime}(y)}{1+\left(\tilde{\Upsilon}^{\prime}(y)\right)^{2}} \sqrt{\frac{-\tilde{q}_{0}^{2}(y) \rho\left(\tilde{q}_{0}^{2}(y)\right)}{\rho\left(\tilde{q}_{0}^{2}(y)\right)+2 \tilde{q}_{0}^{2}(y) \rho^{\prime}\left(\tilde{q}_{0}^{2}(y)\right)}}, \quad 0 \leq y<y_{0} .
\end{gathered}
$$

\subsection{Sufficient conditions for formation of vacuum}

Proposition 4.1 Assume that $f \in C^{2}\left(\left[l_{0}, l_{1}\right)\right)$ satisfies (2.30]), and $Q_{0} \in C^{1}([0, m])$ and $G_{0} \in$ $C([0, m])$ satisfy (2.47) and (2.48). Let $Q \in C^{1}([0, \zeta) \times[0, m])$ with $\zeta \leq+\infty$ be a solution to the problem (2.40)-(2.45). Then

$\arctan f^{\prime}(\check{x})-\arctan f^{\prime}(\hat{x}) \leq \mathscr{H}\left(Q\left(\Phi_{\text {up }}(\check{x}), m\right)\right)-\mathscr{H}\left(Q\left(\Phi_{\text {up }}(\hat{x}), m\right)\right), \quad l_{0} \leq \hat{x} \leq \check{x}<X_{\text {up }}(\zeta)$.

Proof. Theorem 3.1 shows

$$
W(\varphi, \psi) \leq 0, \quad Z(\varphi, \psi) \geq 0, \quad(\varphi, \psi) \in[0, \zeta) \times[0, m]
$$

which, together with (2.54), leads to

$$
\begin{aligned}
Q_{\varphi}(\varphi, m)=\frac{1}{2}(W(\varphi, m)-Z(\varphi, m)) & \leq-\frac{1}{2}(W(\varphi, m)+Z(\varphi, m)) \\
& =-\frac{f^{\prime \prime}\left(X_{\mathrm{up}}(\varphi)\right)\left(1+\left(f^{\prime}\left(X_{\mathrm{up}}(\varphi)\right)\right)^{2}\right)^{-3 / 2}}{b^{1 / 2}(Q(\varphi, m)) A_{+}^{-1}(Q(\varphi, m))}, \quad 0<\varphi<\zeta .
\end{aligned}
$$

Therefore, for any $l_{0} \leq \hat{x} \leq \check{x}<X_{\text {up }}(\zeta)$,

$$
\begin{aligned}
\mathscr{H}\left(Q\left(\Phi_{\mathrm{up}}(\hat{x}), m\right)\right)-\mathscr{H}\left(Q\left(\Phi_{\mathrm{up}}(\check{x}), m\right)\right) & =\int_{\Phi_{\mathrm{up}}(\hat{x})}^{\Phi_{\mathrm{up}}(\check{x})} b^{1 / 2}(Q(\varphi, m)) Q_{\varphi}(\varphi, m) d \varphi \\
& \leq-\int_{\Phi_{\mathrm{up}}(\hat{x})}^{\Phi_{\mathrm{up}}(\check{x})} \frac{f^{\prime \prime}\left(X_{\mathrm{up}}(\varphi)\right)\left(1+\left(f^{\prime}\left(X_{\mathrm{up}}(\varphi)\right)\right)^{2}\right)^{-3 / 2}}{A_{+}^{-1}(Q(\varphi, m))} d \varphi \\
& =-\int_{\hat{x}}^{\check{x}} \frac{f^{\prime \prime}(x)}{1+\left(f^{\prime}(x)\right)^{2}} d x=\arctan f^{\prime}(\hat{x})-\arctan f^{\prime}(\check{x}) .
\end{aligned}
$$

Remark 4.1 In Proposition 4.1, "=" holds if and only if $W(\cdot, m)=0$ in $\left(\Phi_{\text {up }}(\hat{x}), \Phi_{\text {up }}(\check{x})\right)$.

Remark 4.2 Denote

$$
\mathscr{F}(\gamma)=\mathscr{H}(-\infty)=\int_{-\infty}^{0} b^{1 / 2}(s) d s, \quad \gamma>1
$$

Then

$$
\mathscr{F}^{\prime}(\gamma)<0 \text { for each } \gamma>1, \quad \lim _{\gamma \rightarrow 1^{+}} \mathscr{F}(\gamma)=+\infty, \quad \lim _{\gamma \rightarrow+\infty} \mathscr{F}(\gamma)=0, \quad \mathscr{F}(5 / 3)=\pi / 2
$$

Using Proposition 4.1, one can get the following sufficient conditions for formation of vacuum. 
Theorem 4.2 Assume that $f \in C^{2}\left(\left[l_{0}, l_{1}\right)\right)$ satisfies (2.30), $\Upsilon \in C^{2}\left(\left[0, f\left(l_{0}\right)\right]\right)$ satisfies (2.36) and $q_{0} \in C^{1}\left(\left[0, f\left(l_{0}\right)\right]\right)$ satisfies (2.37) -(2.39). If

$$
\arctan f^{\prime}\left(l_{1}\right)-\arctan f^{\prime}\left(l_{0}\right)>\mathscr{H}(-\infty)-\mathscr{H}\left(Q_{0}\left(f\left(l_{0}\right)\right)\right)
$$

then the smooth supersonic flow to the problem (2.31)-(2.35) admits a vacuum. Furthermore, the first vacuum point in the increasing $x$-direction must form at the upper wall before or at

$$
\hat{x}=\inf \left\{x \in\left(l_{0}, l_{1}\right): \arctan f^{\prime}(x)-\arctan f^{\prime}\left(l_{0}\right) \geq \mathscr{H}(-\infty)-\mathscr{H}\left(Q_{0}\left(f\left(l_{0}\right)\right)\right)\right\} .
$$

Remark 4.3 If $q_{0}$ satisfies (3.96) instead of (2.38) in Theorem 4.2, then "> can be relaxed by " $\geq$ " in (4.12) and the first vacuum point in the increasing $x$-direction must form at the upper wall before $\hat{x}$.

Theorem 4.3 Assume that $f \in C^{2}\left(\left[l_{0}, l_{1}\right)\right)$ satisfies (2.30) but is not a linear function, $\Upsilon \in C^{2}\left(\left[0, f\left(l_{0}\right)\right]\right)$ satisfies (2.36) and $q_{0} \in C^{1}\left(\left[0, f\left(l_{0}\right)\right]\right)$ satisfies (2.37) -(2.39). Then, the global smooth supersonic flow to the problem (2.31) (2.35) must admit a vacuum when $q_{0}\left(f\left(l_{0}\right)\right)$ is near $c^{*}$. Furthermore, the first vacuum point in the increasing $x$-direction tends to $\left(x_{*}, f\left(x_{*}\right)\right)$ with $x_{*}=\inf \left\{x \in\left[l_{0}, l_{1}\right): f^{\prime \prime}(x)>0\right\}$ as $q_{0}\left(f\left(l_{0}\right)\right) \rightarrow c^{*}$.

Theorem 4.4 Assume that $f \in C^{2}\left(\left[l_{0}, l_{1}\right)\right)$ satisfies (2.30), $\Upsilon \in C^{2}\left(\left[0, f\left(l_{0}\right)\right]\right)$ satisfies (2.36) and $q_{0} \in C^{1}\left(\left[0, f\left(l_{0}\right)\right]\right)$ satisfies (2.37), (3.96) and (2.39). If

$$
\begin{cases}\lim _{x \rightarrow+\infty} f^{\prime \prime}(x) x^{2 \gamma /(\gamma+1)}=+\infty, & \text { when } l_{1}=+\infty \text { and } \lim _{x \rightarrow+\infty} f^{\prime}(x)<+\infty \\ \lim _{x \rightarrow l_{1}^{-}} \frac{f^{\prime \prime}(x)}{\left(f^{\prime}(x)\right)^{3}} f^{2 \gamma /(\gamma+1)}(x)=+\infty, & \text { when } l_{1} \leq+\infty \text { and } \lim _{x \rightarrow l_{1}^{-}} f^{\prime}(x)=+\infty\end{cases}
$$

then the smooth supersonic flow to the problem (2.31) admits a vacuum.

Proof. We prove the theorem by a contradiction argument and assume that the problem (2.31)(2.35) admits a global supersonic flow without vacuum, or equivalently the problem (2.40)-(2.45) admits a global solution. For any $l_{0} \leq x \leq \check{x}<X_{\text {up }}(\zeta)$, it follows from Proposition 4.1 and Theorem 3.1 that

$$
\begin{aligned}
\arctan f^{\prime}(\check{x})-\arctan f^{\prime}(x) & \leq \mathscr{H}\left(Q\left(\Phi_{\text {up }}(\check{x}), m\right)\right)-\mathscr{H}\left(Q\left(\Phi_{\text {up }}(x), m\right)\right) \\
& <\mathscr{H}(-\infty)-\mathscr{H}\left(Q\left(\Phi_{\text {up }}(x), m\right)\right) \\
& \leq M_{1} \int_{-Q\left(\Phi_{\text {up }}(x), m\right)}^{+\infty} s^{-(\gamma+1) / 2} d s
\end{aligned}
$$

with a positive constant $M_{1}$ independent of $x$ and $\check{x}$. Proposition 3.1 shows

$$
Q\left(\Phi_{\mathrm{up}}(x), m\right) \leq-M_{2}\left(1+\Phi_{\mathrm{up}}(x)\right)^{2 /(\gamma+1)}, \quad x \in\left[l_{0}, l_{1}\right)
$$

with a positive constant $M_{2}$ independent of $x$. The definition of $\Phi_{\text {up }}$ yields

$$
\Phi_{\mathrm{up}}(x) \geq c_{*} \int_{l_{0}}^{x} \sqrt{1+\left(f^{\prime}(s)\right)^{2}} d s \geq c_{*}\left(x-l_{0}\right)+c_{*}\left(f(x)-f\left(l_{0}\right)\right), \quad x \in\left[l_{0}, l_{1}\right) .
$$


Assume that $l_{1}=+\infty$ and $f^{\prime}(+\infty)=\lim _{x \rightarrow+\infty} f^{\prime}(x)<+\infty$, which imply that $f(x)=O(x)$ as $x \rightarrow+\infty$. Letting $\check{x} \rightarrow+\infty$ in (4.14) gives

$$
\arctan f^{\prime}(+\infty)-\arctan f^{\prime}(x) \leq M_{1} \int_{-Q\left(\Phi_{\mathrm{up}}(x), m\right)}^{+\infty} s^{-(\gamma+1) / 2} d s
$$

which, together with (4.15) and (4.16), leads to

$$
\arctan f^{\prime}(+\infty)-\arctan f^{\prime}(x)=O\left(x^{2 /(\gamma+1)-1}\right) \text { as } x \rightarrow+\infty .
$$

However, the first limit in (4.13) shows

$$
\lim _{x \rightarrow+\infty}\left(\arctan f^{\prime}(+\infty)-\arctan f^{\prime}(x)\right) x^{1-2 /(\gamma+1)}=+\infty,
$$

which contradicts (4.17).

Turn to the case when $l_{1} \leq+\infty$ and $\lim _{x \rightarrow l_{1}^{-}} f^{\prime}(x)=+\infty$, which, together with $\lim _{x \rightarrow l_{1}^{-}}(x+f(x))=+\infty$, imply that $\lim _{x \rightarrow l_{1}^{-}} f(x)=+\infty$ and $x=o(f(x))$ as $x \rightarrow l_{1}^{-}$. Letting $\check{x} \rightarrow l_{1}^{-}$in (4.14) gives

$$
\frac{\pi}{2}-\arctan f^{\prime}(x) \leq M_{1} \int_{-Q\left(\Phi_{\mathrm{up}}(x), m\right)}^{+\infty} s^{-(\gamma+1) / 2} d s
$$

which, together with (4.15) and (4.16), leads to

$$
\frac{\pi}{2}-\arctan f^{\prime}(x)=O\left(f^{2 /(\gamma+1)-1}(x)\right) \text { as } x \rightarrow l_{1}^{-} .
$$

However, the second limit in (4.13) shows

$$
\lim _{x \rightarrow l_{1}^{-}}\left(\frac{\pi}{2}-\arctan f^{\prime}(x)\right) f^{1-2 /(\gamma+1)}(x)=+\infty
$$

which contradicts (4.18).

Remark 4.4 Theorem 4.4 still holds if (4.13) is relaxed by

$$
\begin{cases}\lim _{x \rightarrow+\infty}\left(\arctan f^{\prime}(+\infty)-\arctan f^{\prime}(x)\right) x^{1-2 /(\gamma+1)}=+\infty, & \text { when } l_{1}=+\infty \text { and } \lim _{x \rightarrow+\infty} f^{\prime}(x)<+\infty, \\ \lim _{x \rightarrow+\infty}\left(\pi / 2-\arctan f^{\prime}(x)\right) f^{1-2 /(\gamma+1)}(x)=+\infty, & \text { when } l_{1} \leq+\infty \text { and } \lim _{x \rightarrow l_{1}^{-}} f^{\prime}(x)=+\infty .\end{cases}
$$

\subsection{Lower bound estimates of the location of the first vacuum point}

Proposition 4.2 Assume that $f \in C^{2}\left(\left[l_{0}, l_{1}\right)\right)$ satisfies (2.30]), and $Q_{0} \in C^{1}([0, m])$ and $G_{0} \in$ $C([0, m])$ satisfy (2.47) and (2.48). Let $Q \in C^{1}([0, \zeta) \times[0, m])$ with $0<\zeta<+\infty$ be the maximal smooth supersonic flow before vacuum formation to the problem (2.40)-(2.45). Then,

$$
\begin{gathered}
\arctan f^{\prime}\left(X_{\mathrm{up}}(\zeta)\right)>\mathscr{H}(-\infty)-\mathscr{H}\left(\frac { 1 } { m } \left(\int_{0}^{m} Q_{0}(\psi) d \psi+\zeta \int_{0}^{m} G_{0}(\psi) d \psi\right.\right. \\
\left.\left.-c^{*} \int_{l_{0}}^{X_{\mathrm{up}}(\zeta)}\left(\arctan f^{\prime}(x)-\arctan f^{\prime}\left(l_{0}\right)\right) \sqrt{1+\left(f^{\prime}(x)\right)^{2}} d x\right)\right) .
\end{gathered}
$$


Proof. It follows from (2.40)-(2.45) that

$$
\begin{aligned}
\frac{d^{2}}{d \varphi^{2}} \int_{0}^{m} Q(\varphi, \psi) d \psi & =-\frac{f^{\prime \prime}\left(X_{\mathrm{up}}(\varphi)\right)\left(1+\left(f^{\prime}\left(X_{\mathrm{up}}(\varphi)\right)\right)^{2}\right)^{-3 / 2}}{A_{+}^{-1}(Q(\varphi, m))}, \quad \varphi \in(0, \zeta), \\
\left.\int_{0}^{m} Q(\varphi, \psi) d \psi\right|_{\varphi=0} & =\int_{0}^{m} Q_{0}(\psi) d \psi,\left.\quad \frac{d}{d \varphi} \int_{0}^{m} Q(\varphi, \psi) d \psi\right|_{\varphi=0}=\int_{0}^{m} G_{0}(\psi) d \psi .
\end{aligned}
$$

Therefore,

$$
\begin{aligned}
\int_{0}^{m} Q(\zeta, \psi) d \psi=\int_{0}^{m} & Q_{0}(\psi) d \psi+\zeta \int_{0}^{m} G_{0}(\psi) d \psi \\
& -\int_{0}^{\zeta} \int_{0}^{s} \frac{f^{\prime \prime}\left(X_{\mathrm{up}}(\varphi)\right)\left(1+\left(f^{\prime}\left(X_{\mathrm{up}}(\varphi)\right)\right)^{2}\right)^{-3 / 2}}{A_{+}^{-1}(Q(\varphi, m))} d \varphi d s .
\end{aligned}
$$

Direct calculation gives

$$
\begin{aligned}
& \int_{0}^{\zeta} \int_{0}^{s} \frac{f^{\prime \prime}\left(X_{\mathrm{up}}(\varphi)\right)\left(1+\left(f^{\prime}\left(X_{\mathrm{up}}(\varphi)\right)\right)^{2}\right)^{-3 / 2}}{A_{+}^{-1}(Q(\varphi, m))} d \varphi d s \\
= & \int_{l_{0}}^{X_{\mathrm{up}}(\zeta)} A_{+}^{-1}(Q(t, m)) \sqrt{1+\left(f^{\prime}(t)\right)^{2}} \int_{l_{0}}^{t} \frac{f^{\prime \prime}(x)}{1+\left(f^{\prime}(x)\right)^{2}} d x d t \\
< & c^{*} \int_{l_{0}}^{X_{\mathrm{up}}(\zeta)}\left(\arctan f^{\prime}(x)-\arctan f^{\prime}\left(l_{0}\right)\right) \sqrt{1+\left(f^{\prime}(x)\right)^{2}} d x .
\end{aligned}
$$

By (4.20) and (4.21), there exists $\psi_{0} \in(0, m)$ such that

$$
\begin{aligned}
Q\left(\zeta, \psi_{0}\right)>\frac{1}{m}( & \int_{0}^{m} Q_{0}(\psi) d \psi+\zeta \int_{0}^{m} G_{0}(\psi) d \psi \\
& \left.-c^{*} \int_{l_{0}}^{X_{\mathrm{up}}(\zeta)}\left(\arctan f^{\prime}(x)-\arctan f^{\prime}\left(l_{0}\right)\right) \sqrt{1+\left(f^{\prime}(x)\right)^{2}} d x\right) .
\end{aligned}
$$

It follows from Theorem 3.1 that

$$
\frac{\partial}{\partial \psi}(\mathscr{H}(Q(\varphi, \psi))-\theta(\varphi, \psi))=Q_{\varphi}(\varphi, \psi)-b^{1 / 2}(Q(\varphi, \psi)) Q_{\psi}(\varphi, \psi) \leq 0, \quad(\varphi, \psi) \in(0, \zeta) \times(0, m),
$$

which, together with Theorem 4.1, leads to

$$
\mathscr{H}(-\infty)-\arctan f^{\prime}\left(X_{\text {up }}(\zeta)\right) \leq \mathscr{H}\left(Q\left(\zeta, \psi_{0}\right)\right)-\theta\left(\zeta, \psi_{0}\right) \leq \mathscr{H}\left(Q\left(\zeta, \psi_{0}\right)\right) .
$$

Then, (4.19) follows from (4.22) and (4.23).

Theorem 4.5 Assume that $f \in C^{2}\left(\left[l_{0}, l_{1}\right)\right)$ satisfies (2.30) but is not a linear function, $\Upsilon \in C^{2}\left(\left[0, f\left(l_{0}\right)\right]\right)$ satisfies (2.36) and $q_{0} \in C^{1}\left(\left[0, f\left(l_{0}\right)\right]\right)$ satisfies (2.37)-(2.39). Set

$$
\check{x}=\inf \left\{x \in\left(l_{0}, l_{1}\right): \arctan f^{\prime}(x) \geq \mathscr{H}(-\infty)-\mathscr{H}(h(x))\right\},
$$

where for $x \in\left(l_{0}, l_{1}\right)$,

$$
h(x)=\frac{1}{m}\left(\int_{0}^{m} Q_{0}(\psi) d \psi+\zeta \int_{0}^{m} G_{0}(\psi) d \psi-c^{*} \int_{l_{0}}^{x}\left(\arctan f^{\prime}(s)-\arctan f^{\prime}\left(l_{0}\right)\right) \sqrt{1+\left(f^{\prime}(s)\right)^{2}} d s\right) .
$$

Then there is no vacuum on $\left\{(x, f(x)): l_{0} \leq x \leq \check{x}\right\}$ for the global smooth transonic flow to the problem (2.31)-(2.35) . 


\subsection{Formation of a shock in a nozzle whose upper wall is a non-convex perturba- tion of a straight line}

Below, we explain the condition that the upper wall of the nozzle is convex. Indeed, if it is a nonconvex perturbation of a straight line, then there is a $q_{0} \in C^{1}\left(\left[0, f\left(l_{0}\right)\right]\right)$ satisfying (2.37)-(2.39) such that a shock must form for the supersonic flow to the problem (2.31)-(2.35).

Theorem 4.6 Assume that $f \in C^{2}\left(\left[l_{0},+\infty\right)\right)$ satisfies

$$
f\left(l_{0}\right)>0, \quad f^{\prime}\left(l_{0}\right)>0, \quad f^{\prime \prime}\left(l_{0}\right)=0, \quad \sup _{\left(l_{0},+\infty\right)}\left|f^{\prime \prime}\right| \leq \varepsilon, \quad \inf _{\left(l_{0},+\infty\right)} f^{\prime \prime}<0,
$$

and $\Upsilon \in C^{2}\left(\left[0, f\left(l_{0}\right)\right]\right)$ satisfies (2.36) and $\Upsilon^{\prime \prime}(0)=\Upsilon^{\prime \prime}\left(f\left(l_{0}\right)\right)=0$, where $\varepsilon \in(0,1)$ is a constant. Then, there exist a function $q_{0} \in C^{1}\left(\left[0, f\left(l_{0}\right)\right]\right)$ satisfying (2.37)-(2.39) and a positive constant $\varepsilon_{0} \in(0,1)$ such that if $0<\varepsilon<\varepsilon_{0}$, then a shock must form for the global supersonic flow to the problem (2.31)-(2.35).

Proof. Set $l_{*}=\inf \left\{x>l_{0}: f^{\prime \prime}(x)<0\right\}$ and $\zeta_{*}=c^{*}\left(1+f^{\prime}\left(l_{0}\right)+l_{*}-l_{0}\right)\left(l_{*}-l_{0}\right)$. Without loss of generality, it is assumed that $l_{*}>l_{0}$. Choose $0<\delta_{0}<\left(c^{*}-c_{*}\right) / 2$ such that

$$
\zeta_{*} b^{1 / 2}\left(A\left(c^{*}-2 \delta_{0}\right)\right)<m, \quad \mathscr{H}\left(A\left(c^{*}-2 \delta_{0}\right)\right)+\arctan f^{\prime}\left(l_{0}\right)>\mathscr{H}(-\infty) .
$$

For $0<\delta<\delta_{0}$, consider the problem

$$
q_{0}^{\prime}(y)=\frac{-\Upsilon^{\prime \prime}(y)}{1+\left(\Upsilon^{\prime}(y)\right)^{2}} \sqrt{\frac{-q_{0}^{2}(y) \rho\left(q_{0}^{2}(y)\right)}{\rho\left(q_{0}^{2}(y)\right)+2 q_{0}^{2}(y) \rho^{\prime}\left(q_{0}^{2}(y)\right)}}, \quad 0<y<f\left(l_{0}\right), \quad q\left(l_{0}\right)=c^{*}-\delta .
$$

Since

$$
\lim _{s \rightarrow c^{*-}} \sqrt{\frac{-s^{2} \rho\left(s^{2}\right)}{\rho\left(s^{2}\right)+2 s^{2} \rho^{\prime}\left(s^{2}\right)}}=0,
$$

there exists $\delta \in\left(0, \delta_{0}\right)$ such that the problem (4.25) admits a solution $q_{0} \in C^{1}\left(\left[0, f\left(l_{0}\right)\right]\right)$ satisfying $q_{0}(0) \geq c^{*}-\delta_{0}$. Consider the problem (2.31)-(2.35) with this $q_{0}$, which satisfies (2.37)-(2.39). Then, in the potential plane, the boundary data at the inlet satisfy

$$
Q_{0}(\psi) \leq A\left(c^{*}-\delta_{0}\right)<A\left(c^{*}-2 \delta_{0}\right), \quad W_{0}(\psi)=0, \quad Z_{0}(\psi) \geq 0, \quad 0<\psi<m .
$$

Note that $f^{\prime \prime} \geq 0$ on $\left[l_{0}, l_{*}\right]$. According to Theorem 3.1, the problem (2.40)-(2.45) admits uniquely a solution $Q \in C^{1}([0, \zeta) \times[0, m])$ for some $\zeta>0$, where either $\zeta=\Phi_{\mathrm{up}}\left(l_{*}\right)$ or $X_{\mathrm{up}}(\zeta)<l_{*}$ with $\lim _{\varphi \rightarrow \zeta^{-}} \min _{[0, m]} Q(\varphi, \cdot)=-\infty$. Furthermore, the solution satisfies

$$
-Q_{\varphi}(\varphi, \psi) \pm b^{1 / 2}(Q(\varphi, \psi)) Q_{\psi}(\varphi, \psi) \geq 0, \quad Q(\varphi, \psi) \leq A\left(c^{*}-\delta_{0}\right), \quad(\varphi, \psi) \in(0, \zeta) \times(0, m) .
$$

It is clear that $\zeta<\zeta_{*}$. By Theorem 4.1, Proposition 4.1] and Remark4.1, there exists a positive constant $\varepsilon_{0} \in(0,1)$ such that $\zeta=\Phi_{\text {up }}\left(l_{*}\right)$ and $Q \in C^{1}([0, \zeta] \times[0, m])$ for each $0<\varepsilon<\varepsilon_{0}$. Furthermore, (4.24), (4.27) and Lemma 2.1 imply that the positive characteristic from $(\zeta, m)$ must touch $\{0\} \times(0, m)$, while the negative characteristic from $(\zeta, m)$ never touches $(0,+\infty) \times\{0\}$. Take $\tau>0$ sufficiently small such that $Q$ exists on $[\zeta, \zeta+\tau] \times[0, m]$, the positive characteristic from $(\zeta+\tau, m)$ touches $\{0\} \times(0, m)$ and

$$
Q(\varphi, m) \leq A\left(c^{*}-2 \delta_{0}\right), \quad \mathscr{H}\left(A\left(c^{*}-2 \delta_{0}\right)\right) \geq \theta(\zeta, m)-\theta(\varphi, m), \quad \varphi \in(\zeta, \zeta+\tau) .
$$

It follows from (2.49) and the second formula in (4.26) that $W(\cdot, m)=0$ on $[0, \zeta+\tau]$. 


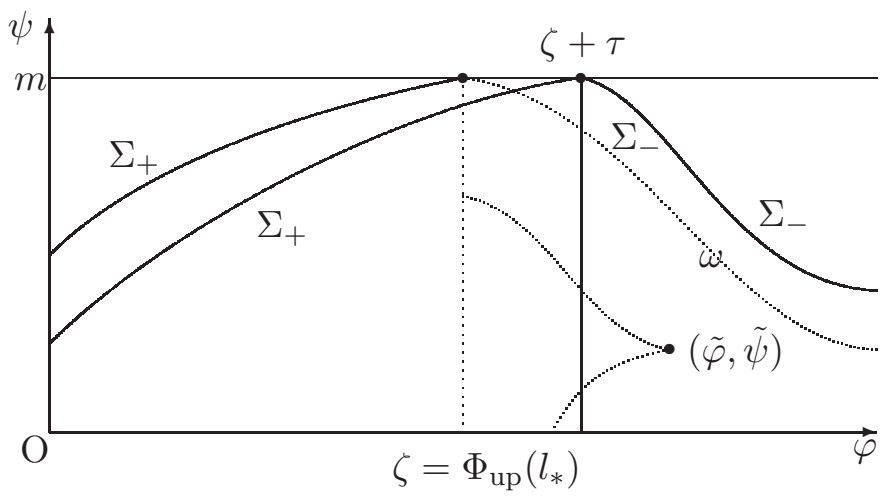

Let $\omega$ be the domain bounded by $\varphi=\zeta+\tau, \psi=0$, and the negative characteristic from $(\zeta+\tau, m)$. Lemma 2.2 gives

$$
-Q_{\varphi}(\varphi, \psi) \leq M_{1}, \quad(\varphi, \psi) \in \omega
$$

with some positive constant $M_{1}$. It follows from Lemma 2.1 and (4.27) that

$$
Q(\varphi, 0) \leq A\left(c^{*}-\delta_{0}\right), \quad \varphi>\zeta .
$$

$\operatorname{Fix}(\tilde{\varphi}, \tilde{\psi}) \in \omega$. Assume that

$$
\tilde{\Sigma}_{ \pm}: \tilde{\Psi}_{ \pm}^{\prime}(\varphi)= \pm b^{1 / 2}\left(Q\left(\varphi, \tilde{\Psi}_{ \pm}(\varphi)\right)\right), \quad \tilde{\varphi}_{ \pm}<\varphi<\tilde{\varphi}, \quad \tilde{\Psi}_{ \pm}\left(\tilde{\varphi}_{ \pm}\right)=\tilde{\psi}_{ \pm}, \quad \tilde{\Psi}_{ \pm}(\tilde{\varphi})=\tilde{\psi}
$$

are the positive and negative characteristics approaching $(\tilde{\varphi}, \tilde{\psi})$, where

$$
\left(\tilde{\varphi}_{+}, \tilde{\psi}_{+}\right) \in\{\zeta\} \times(0, m) \cup[\zeta,+\infty) \times\{0\}, \quad\left(\tilde{\varphi}_{-}, \tilde{\psi}_{-}\right) \in\{\zeta\} \times(0, m) \cup[\zeta, \zeta+\tau] \times\{m\} .
$$

Lemma 2.1 gives

$$
\mathscr{H}\left(Q\left(\tilde{\varphi}_{ \pm}, \tilde{\psi}_{ \pm}\right)\right) \mp \theta\left(\tilde{\varphi}_{ \pm}, \tilde{\psi}_{ \pm}\right)=\mathscr{H}(Q(\tilde{\varphi}, \tilde{\psi})) \mp \theta(\tilde{\varphi}, \tilde{\psi})
$$

Then, (4.27), (4.28), (4.30)-4.32) lead to

$$
\begin{aligned}
2 \mathscr{H}(Q(\tilde{\varphi}, \tilde{\psi})) & =\mathscr{H}\left(Q\left(\tilde{\varphi}_{+}, \tilde{\psi}_{+}\right)\right)+\mathscr{H}\left(Q\left(\tilde{\varphi}_{-}, \tilde{\psi}_{-}\right)\right)-\theta\left(\tilde{\varphi}_{+}, \tilde{\psi}_{+}\right)+\theta\left(\tilde{\varphi}_{-}, \tilde{\psi}_{-}\right) \\
& \geq \mathscr{H}\left(A\left(c^{*}-\delta_{0}\right)\right)+\mathscr{H}\left(A\left(c^{*}-2 \delta_{0}\right)\right)-\theta\left(\tilde{\varphi}_{+}, \tilde{\psi}_{+}\right)+\theta\left(\tilde{\varphi}_{-}, \tilde{\psi}_{-}\right) \geq \mathscr{H}\left(A\left(c^{*}-\delta_{0}\right)\right) .
\end{aligned}
$$

Therefore, there exists a positive constant $M_{2}>0$ such that

$$
Q(\tilde{\varphi}, \tilde{\psi}) \leq-M_{2}, \quad(\tilde{\varphi}, \tilde{\psi}) \in \omega
$$

which, together with (4.29), shows that the flow admits no sonic and vacuum points on $\bar{\omega}$. According to the definition of $l_{*}$, there exists $0<\tilde{\tau}<\tau$ such that $W(\zeta+\tilde{\tau}, m)+Z(\zeta+\tilde{\tau}, m)<0$, which, together with $W(\cdot, m)=0$ on $[0, \zeta+\tau]$, leads to $Z(\zeta+\tilde{\tau}, m)<0$. Then, Lemma 2.4, (4.29) and (4.33) show that a shock must form in $\omega$.

\section{Global smooth supersonic flows}

In this section, we first investigate the properties of a vacuum boundary for a supersonic flow and then consider the dynamics of the vacuum boundary separating the vacuum region from the non-vacuum supersonic flow as a free boundary problem of a smooth supersonic flow from the potential level set where the first vacuum forms. Subsequently, we solve the problem (2.31)-(2.35) globally. Moreover, smooth supersonic flows with vacuum at the inlet are also considered. 


\subsection{Properties of the vacuum boundary}

Generally, the vacuum boundary of a supersonic flow possesses the following properties.

Theorem 5.1 Let $\Omega$ be a bounded domain and $\Gamma \subset \partial \Omega$ be a connected and open $C^{1}$ curve. Assume that $\varphi \in C^{1}(\Omega \cup \Gamma)$ is a supersonic flow such that it is vacuum on $\Gamma$ and there is no vacuum in $\Omega$. Then at each point of $\Gamma$, the flow velocity is along $\Gamma$. Furthermore,

(i) If $\varphi \in C^{2}(\Omega)$ additionally, then $\Gamma$ is a straight line.

(ii) If $\varphi \in C^{2}(\Omega \cup \Gamma)$ additionally, then $\frac{\partial}{\partial \vec{\nu}}|\nabla \varphi|=0$ on $\Gamma$, where $\vec{\nu}$ is the normal to $\Gamma$.

Proof. Assume that there is a point $P_{0} \in \Gamma$ such that at $P_{0}$ the flow velocity is not along the tangent direction of $\Gamma$. Fix a point $\tilde{P}_{0}$, which is located at the different side of $\Gamma$ from $\Omega$, such that the flow velocity at $P_{0}$ is along the direct from $\tilde{P}_{0}$ to $P_{0}$ or its opposite direction. For $r>0$, denote $\Omega_{r}$ to be the set of points which belong to $\Omega$ and whose distances to $\tilde{P}_{0}$ are less than $r$. Since $\varphi \in C^{1}(\Omega \cup \Gamma)$ and $\Gamma \in C^{1}$, there exists a number $r_{0}$, which is greater than the distance from $\tilde{P}_{0}$ to $P_{0}$, such that $\partial \Omega \cap \Omega_{r_{0}} \subset \Gamma$ and for each point $P \in \Omega_{r_{0}}$, the angle between the flow velocity at $P$ and the direction from $\tilde{P}_{0}$ to $P$ is always acute (if the flow velocity at $P_{0}$ is along the direct from $\tilde{P}_{0}$ to $P_{0}$ ) or always obtuse (if the flow velocity at $P_{0}$ is along the direct from $P_{0}$ to $\tilde{P}_{0}$ ). Note that the flow is vacuum on $\Gamma$. Hence

$$
\int_{\partial \Omega_{r} \cap \Omega} \rho\left(|\nabla \varphi(x, y)|^{2}\right) \nabla \varphi(x, y) \cdot \vec{\nu}_{r}(x, y) d \sigma=0, \quad 0<r \leq r_{0},
$$

where $\vec{\nu}_{r}$ is the normal to $\partial \Omega_{r}$. The choice of $r_{0}$ implies that $\nabla \varphi \cdot \vec{\nu}_{r}$ is always positive or always negative on $\partial \Omega_{r} \cap \Omega$ for each $0<r \leq r_{0}$. Therefore, the flow is vacuum on $\bar{\Omega}_{r_{0}}$, which is a contradiction.
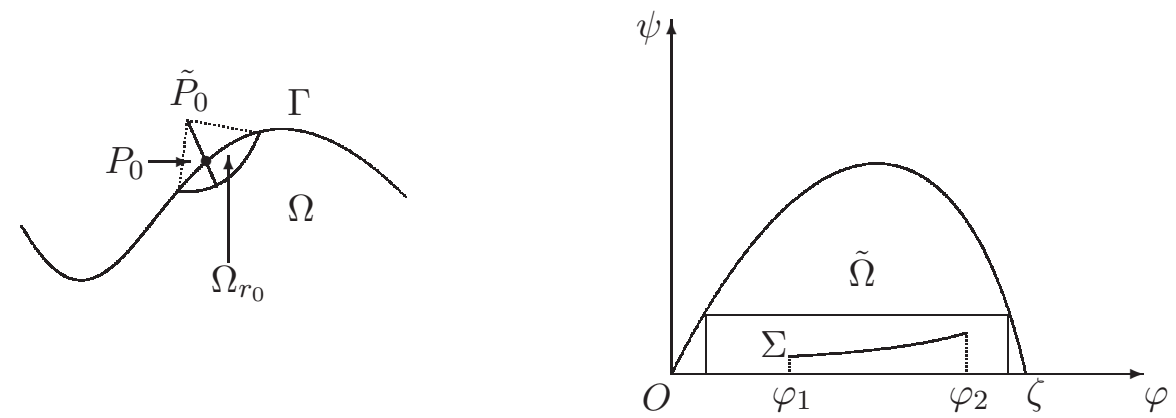

Transform the supersonic flow on $\Omega \cup \Gamma$ into the potential plane. The transformation is one to one since the flow velocity is along $\Gamma$ at each point of $\Gamma$, which also yields that $\Gamma$ is a streamline. Assume that $\Omega$ and $\Gamma$ are transformed into $\tilde{\Omega}$ and $\tilde{\Gamma}$ in the coordinates transformation from the physical plane to the potential plane. Then, $\tilde{\Gamma} \subset \partial \tilde{\Omega}$. Without loss of generality, it is assumed that $\tilde{\Gamma}=(0, \zeta) \times\{0\}$ and $\{\zeta / 2\} \times\left(0, \delta_{0}\right) \subset \tilde{\Omega}$, where $\zeta$ and $\delta_{0}$ are positive constants. For given $0<\varepsilon<\zeta / 2$, there exists a positive constant $\delta<\delta_{0}$ such that $[\varepsilon, \zeta-\varepsilon] \times(0, \delta) \subset \tilde{\Omega}$. Assume that

$$
\Sigma: \Psi^{\prime}(\varphi)=b^{1 / 2}(A(q(\varphi, \Psi(\varphi)))), \quad \varphi_{1}<\varphi<\varphi_{2}, \quad \Psi\left(\varphi_{1}\right)=\psi_{1}
$$

is a positive characteristic in $[\varepsilon, \zeta-\varepsilon] \times(0, \delta)$. Then, for each positive integer $n$,

$$
\Sigma_{n}: \Psi_{n}^{\prime}(\varphi)=b^{1 / 2}\left(A\left(q\left(\varphi, \Psi_{n}(\varphi)\right)\right)\right), \quad \varphi_{1}<\varphi<\varphi_{2}, \quad \Psi_{n}\left(\varphi_{1}\right)=\psi_{1} / n
$$


is also a positive characteristic in $[\varepsilon, \zeta-\varepsilon] \times(0, \delta)$, and

$$
\lim _{n \rightarrow+\infty} \Psi_{n}(\varphi)=0, \quad \varphi_{1} \leq \varphi \leq \varphi_{2} .
$$

For each positive integer $n, \varphi \in C^{2}(\Omega)$ and Lemma 2.1 yield

$$
\mathscr{H}\left(A\left(q\left(\varphi, \Psi_{n}(\varphi)\right)\right)\right)-\theta\left(\varphi, \Psi_{n}(\varphi)\right)=M_{n}, \quad \varphi_{1} \leq \varphi \leq \varphi_{2}
$$

with $M_{n}$ being a constant. Note that

$$
\lim _{\psi \rightarrow 0^{+}} q(\varphi, \psi)=c^{*}, \quad \varphi_{1} \leq \varphi \leq \varphi_{2}
$$

Then, (5.1) - (5.3) and $\varphi \in C^{1}(\Omega \cup \Gamma)$ lead to that $\theta(\cdot, 0)$ is invariant on $\left[\varphi_{1}, \varphi_{2}\right]$. Since $\Sigma$ and $\varepsilon$ are arbitrary, $\theta(\cdot, 0)$ is equal to a constant identically in $(0, \zeta)$. That is to say, the flow angle is invariant on $\Gamma$, which, together with $\Gamma$ being a streamline, implies that $\Gamma$ is a straight line.

Assume that $\varepsilon$ and $\delta$ are given as above. For each $\xi \in C_{0}^{\infty}(\varepsilon, \zeta-\varepsilon)$, one gets from $\varphi \in C^{2}(\Omega \cup \Gamma)$ and (i) that

$$
\begin{aligned}
& \int_{\varepsilon}^{\zeta-\varepsilon} \frac{\partial B(q)}{\partial \psi}(\varphi, 0) \xi(\varphi) d \varphi=\lim _{\psi \rightarrow 0^{+}} \int_{\varepsilon}^{\zeta-\varepsilon} \frac{\partial B(q)}{\partial \psi}(\varphi, \psi) \xi(\varphi) d \varphi \\
= & \lim _{\psi \rightarrow 0^{+}} \int_{\varepsilon}^{\zeta-\varepsilon} \frac{\partial \theta}{\partial \varphi}(\varphi, \psi) \xi(\varphi) d \varphi=-\lim _{\psi \rightarrow 0^{+}} \int_{\varepsilon}^{\zeta-\varepsilon} \theta(\varphi, \psi) \xi^{\prime}(\varphi) d \varphi=0 .
\end{aligned}
$$

Then, it follows from the arbitrariness of $\xi$ and $\varepsilon$ that

$$
\frac{\partial B(q)}{\partial \psi}(\varphi, 0)=0, \quad 0<\varphi<\zeta
$$

which yields (ii) by the transformation from the potential plane to the physical plane.

Remark 5.1 Theorem 5.1 shows that the vacuum of a $C^{1}$ supersonic potential flow is never the so called physical vacuum in [9, 12]. The reason lies in that there is not an external force on the flow.

\subsection{Dynamics of the vacuum boundary and a free boundary problem}

In this subsection, we study the dynamic of the vacuum boundary from its first formation in the potential plane and treat it as a free boundary problem to extend globally a supersonic flow from the potential level set where the first vacuum forms. Assume that $\tilde{Q}_{0} \in C^{1}([0, m))$ and $\tilde{G}_{0} \in C([0, m)) \cap$ $L^{1}(0, m)$ satisfy (4.8) and (4.9). For $0<n<m$, choose $\tilde{Q}_{0, n} \in C^{1}([0, m])$ and $\tilde{G}_{0, n} \in C([0, m])$ to satisfy $\tilde{Q}_{0, n}^{\prime}(m)=0$ and

$$
\begin{gathered}
\tilde{Q}_{0, n}(\psi)=\tilde{Q}_{0}(\psi), \quad \tilde{G}_{0, n}(\psi)=\tilde{G}_{0}(\psi), \quad \psi \in[0, n] \\
\tilde{Q}_{0, n}(\psi)<0, \quad-\tilde{G}_{0, n}(\psi) \pm b^{1 / 2}\left(\tilde{Q}_{0, n}(\psi)\right) \tilde{Q}_{0, n}^{\prime}(\psi) \geq 0, \quad \psi \in[n, m] .
\end{gathered}
$$

Consider the problem

$$
\begin{array}{ll}
\frac{\partial^{2} Q_{n}}{\partial \varphi^{2}}-\frac{\partial}{\partial \psi}\left(b\left(Q_{n}\right) \frac{\partial Q_{n}}{\partial \psi}\right)=0, & (\varphi, \psi) \in(\zeta,+\infty) \times(0, m), \\
Q_{n}(\zeta, \psi)=\tilde{Q}_{0, n}(\psi), & \psi \in(0, m),
\end{array}
$$




$$
\begin{array}{lll}
\frac{\partial Q_{n}}{\partial \varphi}(\zeta, \psi)=\tilde{G}_{0, n}(\psi), & \psi \in(0, m), \\
\frac{\partial Q_{n}}{\partial \psi}(\varphi, 0)=\frac{\partial Q_{n}}{\partial \psi}(\varphi, m)=0, & & \varphi \in(\zeta,+\infty) .
\end{array}
$$

It follows from Theorem 3.3 that the problem (5.4) - (5.7) admits a solution $Q_{n} \in C^{1}([\zeta,+\infty) \times[0, m])$ satisfying

$$
-\frac{\partial Q_{n}}{\partial \varphi}(\varphi, \psi) \pm b^{1 / 2}\left(Q_{n}(\varphi, \psi)\right) \frac{\partial Q_{n}}{\partial \psi}(\varphi, \psi) \geq 0, \quad(\varphi, \psi) \in(\zeta,+\infty) \times(0, m) .
$$

Lemma 5.1 Let $0<k \leq n<m$. Assume that

$$
\Sigma_{n}:\left\{\begin{array}{l}
\Psi_{n}^{\prime}(\varphi)=-b^{1 / 2}\left(Q_{n}\left(\varphi, \Psi_{n}(\varphi)\right)\right), \quad 0<\Psi_{n}(\varphi)<k, \quad \zeta<\varphi<\varphi_{n} \leq+\infty, \\
\Psi_{n}(\zeta)=k, \quad \Psi_{n}\left(\varphi_{n}\right)=0 \text { if } \varphi_{n}<+\infty
\end{array}\right.
$$

is the negative characteristic from $(\zeta, k)$. Then $\varphi_{n} \geq \zeta+b^{-1 / 2}\left(\tilde{Q}_{0}(k)\right) k$ and

$$
\Psi_{n}(\varphi) \geq k-b^{1 / 2}\left(\tilde{Q}_{0}(k)\right)(\varphi-\zeta), \quad \zeta \leq \varphi \leq \zeta+b^{-1 / 2}\left(\tilde{Q}_{0}(k)\right) k .
$$

Proof. On $\Sigma_{n}, Q_{n}$ satisfies

$$
\frac{d}{d \varphi} Q_{n}\left(\varphi, \Psi_{n}(\varphi)\right)=\frac{\partial Q_{n}}{\partial \varphi}\left(\varphi, \Psi_{n}(\varphi)\right)-b^{1 / 2}\left(Q_{n}\left(\varphi, \Psi_{n}(\varphi)\right)\right) \frac{\partial Q_{n}}{\partial \psi}\left(\varphi, \Psi_{n}(\varphi)\right) \leq 0, \quad \zeta<\varphi<\varphi_{n}
$$

according to (5.8). Therefore,

$$
Q_{n}\left(\varphi, \Psi_{n}(\varphi)\right) \leq Q_{n}\left(\zeta, \Psi_{n}(\zeta)\right)=\tilde{Q}_{0, n}(k)=\tilde{Q}_{0}(k), \quad \zeta<\varphi<\varphi_{n},
$$

which implies $\Psi_{n}^{\prime} \geq-b^{1 / 2}\left(\tilde{Q}_{0}(k)\right)$ in $\left(\zeta, \varphi_{n}\right)$ and thus the lemma is proved.

For $0<k \leq n<m$, Lemma 5.1 shows

$$
Q_{n}(\varphi, \psi)=Q_{k}(\varphi, \psi), \quad \zeta \leq \varphi \leq \zeta+b^{-1 / 2}\left(\tilde{Q}_{0}(k)\right) k, 0 \leq \psi \leq k-b^{1 / 2}\left(\tilde{Q}_{0}(k)\right)(\varphi-\zeta) .
$$

By (5.9) and (4.9), one can set

$$
Q(\varphi, \psi)=\lim _{n \rightarrow m^{-}} Q_{n}(\varphi, \psi), \quad(\varphi, \psi) \in[\zeta,+\infty) \times[0, m) .
$$

Proposition 5.1 Assume that $\tilde{Q}_{0} \in C^{1}([0, m))$ and $\tilde{G}_{0} \in C([0, m)) \cap L^{1}(0, m)$ satisfy (4.8) and (4.9)). Then the function $Q$ defined by (5.10) belongs to $C^{1}([\zeta,+\infty) \times[0, m))$ and is the unique solution to the problem

$$
\begin{array}{ll}
Q_{\varphi \varphi}-\left(b(Q) Q_{\psi}\right)_{\psi}=0, & (\varphi, \psi) \in(\zeta,+\infty) \times(0, m), \\
Q(\zeta, \psi)=\tilde{Q}_{0}(\psi), & \psi \in(0, m), \\
Q_{\varphi}(\zeta, \psi)=\tilde{G}_{0}(\psi), & \psi \in(0, m), \\
Q_{\psi}(\varphi, 0)=0, & \varphi \in(\zeta,+\infty) .
\end{array}
$$

Furthermore,

$$
-Q_{\varphi}(\varphi, \psi) \pm b^{1 / 2}(Q(\varphi, \psi)) Q_{\psi}(\varphi, \psi) \geq 0, \quad(\varphi, \psi) \in(\zeta,+\infty) \times(0, m),
$$

and $q, \theta \in C([\zeta,+\infty) \times[0, m])$ satisfying

$$
\begin{gathered}
q(\varphi, \psi)<c^{*}, \quad \theta(\varphi, \psi)<\theta(\zeta, m), \quad(\varphi, \psi) \in[\zeta,+\infty) \times[0, m), \\
\lim _{\psi \rightarrow m^{-}} q(\varphi, \psi)=c^{*} \text { and } \lim _{\psi \rightarrow m^{-}} \theta(\varphi, \psi)=\theta(\zeta, m) \text { uniformly for } \varphi \geq \zeta .
\end{gathered}
$$


Proof. It follows from Lemma 5.1 and (4.9) that $Q \in C^{1}([\zeta,+\infty) \times[0, m))$ is the unique solution to the problem (5.11) -(5.14), and (5.15) follows from (5.8). The first estimate in (5.16) is clear since $Q \in C^{1}([\zeta,+\infty) \times[0, m))$. Moreover, (4.9) and (5.15) lead to the first limit in (5.17).

We now show

$$
\theta(\zeta, \psi)<\theta(\zeta, m), \quad 0<\psi<m
$$

by a contradiction argument. Otherwise, by (4.8), there exists $\psi_{0} \in(0, m)$ such that $\theta(\zeta, \cdot)=\theta(\zeta, m)$ in $\left(\psi_{0}, m\right)$, which yields $\tilde{G}_{0}=0$ in $\left(\psi_{0}, m\right)$. Then, (4.8) implies $\tilde{Q}_{0}^{\prime}=0$ in $\left(\psi_{0}, m\right)$, which contradicts the second formula in (4.9) and thus (5.18) is proved.

For $0<n<m$, set

$$
\Sigma_{n}:\left\{\begin{array}{l}
\Psi_{n}^{\prime}(\varphi)=-b^{1 / 2}\left(Q\left(\varphi, \Psi_{n}(\varphi)\right)\right), \quad 0<\Psi_{n}(\varphi)<n, \quad \zeta<\varphi<\varphi_{n} \leq+\infty \\
\Psi_{n}(\zeta)=n, \quad \Psi_{n}\left(\varphi_{n}\right)=0 \text { if } \varphi_{n}<+\infty
\end{array}\right.
$$

to be the negative characteristic from $(\zeta, n)$. Lemma 2.1 shows

$$
\mathscr{H}\left(\tilde{Q}_{0}(n)\right)+\theta(\zeta, n)=\mathscr{H}\left(Q\left(\varphi, \Psi_{n}(\varphi)\right)\right)+\theta\left(\varphi, \Psi_{n}(\varphi)\right), \quad \zeta<\varphi<\varphi_{n}
$$

The same proof as Lemma 5.1 gives

$$
Q\left(\varphi, \Psi_{n}(\varphi)\right) \leq \tilde{Q}_{0}(n), \quad \zeta<\varphi<\varphi_{n} .
$$

It follows from (5.19), (5.20) and (5.18) that

$$
\theta\left(\varphi, \Psi_{n}(\varphi)\right) \leq \theta(\zeta, n)<\theta(\zeta, m), \quad \zeta<\varphi<\varphi_{n},
$$

which, together with (5.20) and (4.9), leads to the second estimate in (5.16). Furthermore, the second limit in (5.17) follows from (5.19), (5.20) and (4.9). Finally, (5.17) shows $q, \theta \in C([\zeta,+\infty) \times[0, m])$.

In the physical plane, Proposition 5.1 is stated as follows.

Proposition 5.2 Assume that $\tilde{\Upsilon} \in C^{2}\left(\left[0, y_{0}\right)\right) \cap C^{1}\left(\left[0, y_{0}\right]\right)$ and $\tilde{q}_{0} \in C^{1}\left(\left[0, y_{0}\right)\right) \cap C\left(\left[0, y_{0}\right]\right)$ satisfy (4.10) and (4.11). Then the problem

$$
\begin{array}{ll}
\operatorname{div}\left(\rho\left(|\nabla \varphi|^{2}\right) \nabla \varphi\right)=0, & (x, y) \in \tilde{\Omega}, \\
\varphi(\tilde{\Upsilon}(y), y)=\zeta, & 0<y<y_{0}, \\
|\nabla \varphi(\tilde{\Upsilon}(y), y)|=\tilde{q}_{0}(y), & 0<y<y_{0}, \\
\frac{\partial \varphi}{\partial y}(x, 0)=0, & x>\tilde{\Upsilon}(0)
\end{array}
$$

admits a unique solution $\varphi \in C^{2}\left(\overline{\tilde{\Omega}} \backslash \tilde{\Gamma}_{\text {up }}\right) \cap C^{1}(\overline{\tilde{\Omega}})$, where

$$
\tilde{\Gamma}_{\text {up }}=\left\{(x, y): x \geq \tilde{\Upsilon}\left(y_{0}\right), y=y_{0}-\tilde{\Upsilon}^{\prime}\left(y_{0}\right)\left(x-\tilde{\Upsilon}\left(y_{0}\right)\right)\right\}
$$

and $\tilde{\Omega}$ is the domain bounded by $\left\{(\tilde{\Upsilon}(y), y): 0 \leq y \leq y_{0}\right\}, \tilde{\Gamma}_{\text {up }}$ and the $x$-axis. Furthermore, $\varphi$ satisfies

$$
\inf _{\left(0, y_{0}\right)} \tilde{q}_{0} \leq|\nabla \varphi(x, y)|<c^{*}, \quad 0 \leq \frac{\partial \varphi}{\partial y}(x, y)<-\tilde{\Upsilon}^{\prime}\left(y_{0}\right) \frac{\partial \varphi}{\partial x}(x, y), \quad(x, y) \in \overline{\tilde{\Omega}} \backslash \tilde{\Gamma}_{\mathrm{up}}
$$




$$
\begin{gathered}
|\nabla \varphi(x, y)|=c^{*}, \quad \frac{\partial \varphi}{\partial x}(x, y)=\frac{c^{*}}{\sqrt{1+\left(\tilde{\Upsilon}^{\prime}\left(y_{0}\right)\right)^{2}}}, \quad \frac{\partial \varphi}{\partial y}(x, y)=\frac{-\tilde{\Upsilon}^{\prime}\left(y_{0}\right) c^{*}}{\sqrt{1+\left(\tilde{\Upsilon}^{\prime}\left(y_{0}\right)\right)^{2}}}, \quad(x, y) \in \tilde{\Gamma}_{\mathrm{up}}, \\
\varphi(x, y)=\zeta+\frac{c^{*}}{\sqrt{1+\left(\tilde{\Upsilon}^{\prime}\left(y_{0}\right)\right)^{2}}}\left(\left(x-\tilde{\Upsilon}\left(y_{0}\right)\right)-\tilde{\Upsilon}^{\prime}\left(y_{0}\right)\left(y-y_{0}\right)\right), \quad(x, y) \in \tilde{\Gamma}_{\mathrm{up}} .
\end{gathered}
$$

Below we study the global behavior of positive characteristics and the lower bound estimate of the flow speed to the problem (5.11)-(5.14).

Proposition 5.3 Assume that $Q \in C^{1}([\zeta,+\infty) \times[0, m))$ is the solution to the problem (5.11) -(5.14), where $\tilde{Q}_{0} \in C^{1}([0, m))$ and $\tilde{G}_{0} \in C([0, m)) \cap L^{1}(0, m)$ satisfying (4.8) and (4.9). Then, each positive characteristic never touches $(\zeta,+\infty) \times\{m\}$. Furthermore, if, in addition, $\inf _{(0, m)}\left(-\tilde{G}_{0}-b^{1 / 2}\left(\tilde{Q}_{0}\right)\left|\tilde{Q}_{0}^{\prime}\right|\right)>$ 0 , then

$$
Q(\varphi, \psi) \leq-M(\varphi+1)^{2 /(\gamma+1)}, \quad(\varphi, \psi) \in(\zeta,+\infty) \times(0, m),
$$

where $M>0$ is a constant depending only on $\gamma, m, \sup _{(0, m)} \tilde{Q}_{0}$ and $\inf _{(0, m)}\left(-\tilde{G}_{0}-b^{1 / 2}\left(\tilde{Q}_{0}\right)\left|\tilde{Q}_{0}^{\prime}\right|\right)$.

Proof. For $(\varphi, \psi) \in[\zeta,+\infty) \times[0, m)$, set

$$
W(\varphi, \psi)=Q_{\varphi}(\varphi, \psi)-b^{1 / 2}(Q(\varphi, \psi)) Q_{\psi}(\varphi, \psi), \quad Z(\varphi, \psi)=-Q_{\varphi}(\varphi, \psi)-b^{1 / 2}(Q(\varphi, \psi)) Q_{\psi}(\varphi, \psi) .
$$

Then, $(W, Z) \in C([\zeta,+\infty) \times[0, m)) \times C([\zeta,+\infty) \times[0, m))$ solves

$$
\begin{array}{ll}
W_{\varphi}+b^{1 / 2}(Q) W_{\psi}=\frac{1}{4} b^{-1}(Q) p(Q) W(W+Z), & (\varphi, \psi) \in(\zeta,+\infty) \times(0, m), \\
Z_{\varphi}-b^{1 / 2}(Q) Z_{\psi}=-\frac{1}{4} b^{-1}(Q) p(Q) Z(W+Z), & (\varphi, \psi) \in(\zeta,+\infty) \times(0, m) .
\end{array}
$$

It follows from (5.15) that

$$
W(\varphi, \psi) \leq 0, \quad Z(\varphi, \psi) \geq 0, \quad(\varphi, \psi) \in[\zeta,+\infty) \times[0, m) .
$$

First, consider the case that $m \in \operatorname{supp} W(\zeta, \cdot)$. Note that

$$
\frac{d}{d \psi}(\mathscr{H}(Q(\zeta, \psi))-\theta(\zeta, \psi))=Q_{\varphi}(\zeta, \psi)-b^{1 / 2}(Q(\zeta, \psi)) Q_{\psi}(\zeta, \psi)=W(\zeta, \psi), \quad 0<\psi<m,
$$

which, together with (5.24), $m \in \operatorname{supp} W(\zeta, \cdot)$ and (5.17), leads to

$$
\mathscr{H}(Q(\zeta, \psi))-\theta(\zeta, \psi)>\mathscr{H}(-\infty)-\theta(\zeta, m), \quad 0<\psi<m .
$$

Then, Lemma 2.1 and (5.17) show that for each $\psi \in(0, m)$, the positive characteristic from $(\zeta, \psi)$ never touches $(\zeta,+\infty) \times\{m\}$.

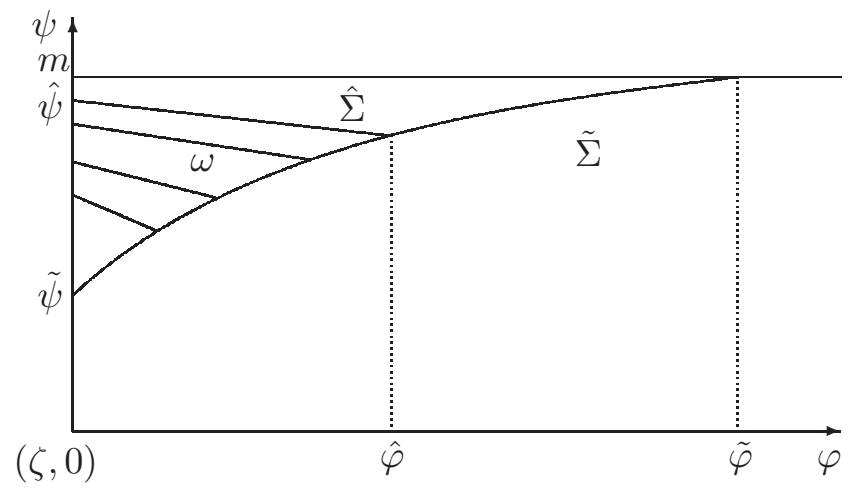


Next, consider the case that $m \notin \operatorname{supp} W(\zeta, \cdot)$, which, together with (5.24), implies that there exists $\psi_{0} \in(0, m)$ such that

$$
W(\zeta, \psi)=0, \quad \psi_{0}<\psi<m
$$

It can be proved by a contradiction argument that each positive characteristic never touches $(\zeta,+\infty) \times$ $\{m\}$. Otherwise, there exists $\tilde{\psi} \in\left(\psi_{0}, m\right)$ such that the positive characteristic $\tilde{\Sigma}$ from $(\zeta, \tilde{\psi})$ touches $(\zeta,+\infty) \times\{m\}$. That is to say,

$$
\tilde{\Sigma}: \tilde{\Psi}^{\prime}(\varphi)=b^{1 / 2}(Q(\varphi, \tilde{\Psi}(\varphi))), \quad \tilde{\psi}<\tilde{\Psi}(\varphi)<m, \quad \zeta<\varphi<\tilde{\varphi}, \quad \tilde{\Psi}(\zeta)=\tilde{\psi}, \quad \tilde{\Psi}(\tilde{\varphi})=m .
$$

Let $\omega$ be the bounded domain bounded by $\varphi=\zeta, \psi=m$ and $\tilde{\Sigma}$. Then, (5.22) and (15.25) yield that

$$
W(\varphi, \psi)=0, \quad(\varphi, \psi) \in \omega
$$

For each $\hat{\psi} \in(\tilde{\psi}, m)$, let

$$
\hat{\Sigma}: \hat{\Psi}^{\prime}(\varphi)=-b^{1 / 2}(Q(\varphi, \hat{\Psi}(\varphi))), \quad \zeta<\varphi<\hat{\varphi}, \quad \hat{\Psi}(\zeta)=\hat{\psi}, \quad \hat{\Psi}(\hat{\varphi})=\tilde{\Psi}(\hat{\varphi})
$$

be the negative characteristic from $(\zeta, \hat{\psi})$ to $\tilde{\Sigma}$. It follows from (5.26) that

$$
\frac{d}{d \varphi} Q(\varphi, \hat{\Psi}(\varphi))=W(\varphi, \hat{\Psi}(\varphi))=0, \quad \zeta<\varphi<\hat{\varphi}
$$

which yields that $\hat{\Sigma}$ is a straight line and

$$
Q(\varphi, \hat{\Psi}(\varphi)))=Q(\hat{\varphi}, \hat{\Psi}(\hat{\varphi})), \quad \zeta<\varphi<\hat{\varphi} .
$$

By (5.23), (5.26), (5.27) and (2.10), there exists a positive constant $M$ depending only on $\gamma$ such that

$$
\begin{aligned}
\frac{d}{d \varphi} Z(\varphi, \hat{\Psi}(\varphi)) & =-\frac{1}{4} b^{-1}(Q(\varphi, \hat{\Psi}(\varphi))) p(Q(\varphi, \hat{\Psi}(\varphi))) Z^{2}(\varphi, \hat{\Psi}(\varphi)) \\
& \leq M Q^{-1}(\varphi, \hat{\Psi}(\varphi)) Z^{2}(\varphi, \hat{\Psi}(\varphi))=M Q^{-1}(\hat{\varphi}, \hat{\Psi}(\hat{\varphi})) Z^{2}(\varphi, \hat{\Psi}(\varphi)), \quad \zeta<\varphi<\hat{\varphi}
\end{aligned}
$$

which, together with (5.24) and $\hat{\Psi}(\hat{\varphi})=\tilde{\Psi}(\hat{\varphi})$, leads to

$$
Z(\hat{\varphi}, \tilde{\Psi}(\hat{\varphi})) \leq-\frac{Q(\hat{\varphi}, \tilde{\Psi}(\hat{\varphi}))}{M(\hat{\varphi}-\zeta)}
$$

By (5.17), $\hat{\varphi}$ will take all values over $(\zeta, \tilde{\varphi})$ when $\hat{\psi}$ varies from $\tilde{\psi}$ to $m$. Therefore,

$$
\frac{d}{d \varphi} Q(\varphi, \tilde{\Psi}(\varphi))=-Z(\varphi, \tilde{\Psi}(\varphi)) \geq \frac{2}{M(\tilde{\varphi}-\zeta)} Q(\varphi, \tilde{\Psi}(\varphi)), \quad \frac{\zeta+\tilde{\varphi}}{2}<\varphi<\tilde{\varphi}
$$

which contradicts $\tilde{\Psi}(\tilde{\varphi})=m$ and (5.17).

Summing up, one gets that each positive characteristic never touches $(\zeta,+\infty) \times\{m\}$. Then, (5.21) can be proved by a similar discussion as Step III in the proof of Proposition 3.1. 


\subsection{Global smooth supersonic flows}

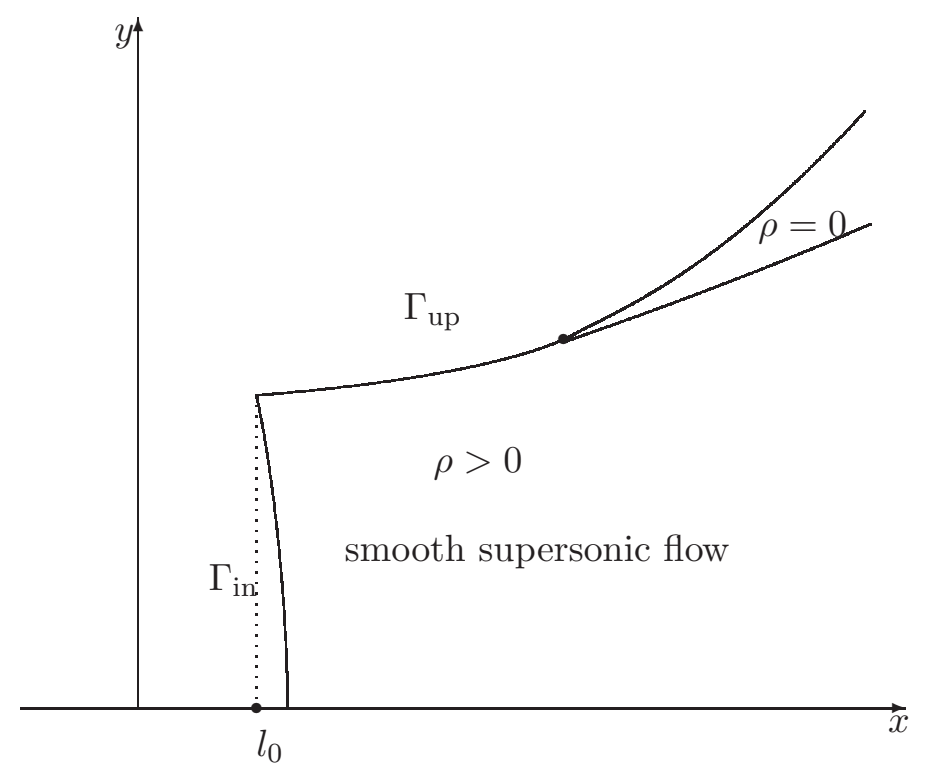

Theorem 5.2 Assume that $f \in C^{2}\left(\left[l_{0}, l_{1}\right)\right)$ satisfies (2.30), $\Upsilon \in C^{2}\left(\left[0, f\left(l_{0}\right)\right]\right)$ satisfies (2.36) and $q_{0} \in C^{1}\left(\left[0, f\left(l_{0}\right)\right]\right)$ satisfies (2.37)-(2.39). Then the problem (2.31) -(2.35) admits uniquely a global smooth solution $\varphi \in C^{1}(\bar{\Omega})$ with $|\nabla \varphi| \in C^{0,1}(\bar{\Omega})$. Moreover, the global smooth supersonic flow belongs to one and only one of the following two cases:

Case I Global smooth supersonic flow without vacuum. In this case, $\varphi \in C^{2}(\bar{\Omega})$ and $c_{1} \leq|\nabla \varphi|<$ $c^{*}$ on $\bar{\Omega}$. If $f^{\prime \prime}\left(1+\left(f^{\prime}\right)^{2}\right)^{-3 / 2} \in L^{\infty}\left(l_{0}, l_{1}\right)$ and $q_{0}$ satisfies (3.96) additionally, then $|\nabla \varphi|$ is globally Lipschitz continuous in $\Omega$.

Case II Global smooth supersonic flow with vacuum. In this case, set

$$
x_{0}=\sup \left\{l \in\left(l_{0}, l_{1}\right):|\nabla \varphi(x, f(x))|<c^{*} \text { for each } l_{0}<x<l\right\}
$$

and

$$
\Omega_{\mathrm{v}}=\left\{(x, y) \in \Omega: x>x_{0}, y>f\left(x_{0}\right)+f^{\prime}\left(x_{0}\right)\left(x-x_{0}\right)\right\} .
$$

Then

(i) $\bar{\Omega}_{\mathrm{V}}$ is the set of vacuum points. Moreover, $\varphi \in C^{2}\left(\bar{\Omega} \backslash \bar{\Omega}_{\mathrm{V}}\right)$ satisfying

$$
\begin{gathered}
c_{1} \leq|\nabla \varphi(x, y)|<c^{*}, \quad 0 \leq \frac{\partial \varphi}{\partial y}(x, y)<f^{\prime}\left(x_{0}\right) \frac{\partial \varphi}{\partial x}(x, y), \quad(x, y) \in \bar{\Omega} \backslash \bar{\Omega}_{\mathrm{v}}, \\
\frac{\partial \varphi}{\partial x}(x, y)=\frac{c^{*}}{\sqrt{1+\left(f^{\prime}\left(x_{0}\right)\right)^{2}}}, \quad \frac{\partial \varphi}{\partial y}(x, y)=\frac{f^{\prime}\left(x_{0}\right) c^{*}}{\sqrt{1+\left(f^{\prime}\left(x_{0}\right)\right)^{2}}}, \quad(x, y) \in \partial \Omega_{\mathrm{v}} \cap \Omega .
\end{gathered}
$$

(ii) $|\nabla \varphi(x, f(x))|=c^{*}-O\left(\left(x_{0}-x\right)^{2}\right)$ as $x \rightarrow x_{0}^{-}$.

(iii) $|\nabla \varphi|$ is globally Lipschitz continuous in $\Omega$.

(iv) $|\nabla \varphi| \in C^{1}\left(\bar{\Omega} \backslash\left(x_{0}, f\left(x_{0}\right)\right)\right)$ and for each $(\tilde{x}, \tilde{y}) \in \partial \Omega_{\mathrm{v}} \cap \Omega$,

$$
\lim _{\substack{(x, y) \rightarrow(\tilde{x}, \tilde{y}) \\(x, y) \in \Omega \backslash \Omega_{\mathrm{v}}}} \nabla|\nabla \varphi(x, y)|=(0,0), \quad \lim _{\substack{(x, y) \rightarrow(\tilde{x}, \tilde{y}) \\(x, y) \in \Omega \backslash \Omega_{\mathrm{v}}}}\left(c^{*}-|\nabla \varphi(x, y)|\right)^{1 / 2} \nabla \arctan \frac{\varphi_{y}(x, y)}{\varphi_{x}(x, y)}=(0,0) .
$$


In particular, if $1<\gamma \leq 2$, then $\rho\left(|\nabla \varphi|^{2}\right) \nabla \varphi \in C^{1}\left(\bar{\Omega} \backslash\left(x_{0}, f\left(x_{0}\right)\right)\right)$.

(v) If $1<\gamma<3$ and $f^{\prime \prime}\left(x_{0}\right)>0$ additionally, then for any $\varepsilon>0$,

$$
\lim _{\substack{(x, y) \rightarrow(\tilde{x}, \tilde{y}) \\(x, y) \in \Omega \backslash \Omega_{\mathrm{v}}}}\left(c^{*}-|\nabla \varphi(x, y)|\right)^{-(\gamma+1+\varepsilon) /(4 \gamma-4)} \nabla|\nabla \varphi(x, y)| \cdot\left(-1, f^{\prime}\left(x_{0}\right)\right)=+\infty, \quad(\tilde{x}, \tilde{y}) \in \partial \Omega_{\mathrm{V}} \cap \Omega .
$$

Proof. If there is no vacuum for the flow, then the theorem is proved by Theorems 3.2 and 3.5 , Below we consider the case that there is a vacuum for the flow. It follows from Theorem 3.2 and Proposition 5.2 that $\bar{\Omega}_{\mathrm{v}}$ is the set of vacuum points for a global smooth supersonic flow to the problem (2.31) - 2.35), and the problem (2.31)-2.35) in the gas region admits uniquely a smooth solution $\varphi \in C^{2}\left(\bar{\Omega} \backslash \bar{\Omega}_{\mathrm{V}}\right) \cap C^{1}\left(\overline{\Omega \backslash \Omega_{\mathrm{V}}}\right)$. By Theorem 3.2 and Proposition 5.2 , $\varphi$ satisfies

$$
\begin{array}{ll}
\operatorname{div}\left(\rho\left(|\nabla \varphi|^{2}\right) \nabla \varphi\right)=0, & (x, y) \in \hat{\Omega}, \\
\varphi(\Upsilon(y), y)=0, & 0<y<f\left(l_{0}\right), \\
|\nabla \varphi(\Upsilon(y), y)|=q_{0}(y), & 0<y<f\left(l_{0}\right), \\
\frac{\partial \varphi}{\partial y}(x, 0)=0, & \Upsilon(0)<x<l_{1}, \\
\frac{\partial \varphi}{\partial y}(x, f(x))-f^{\prime}(x) \frac{\partial \varphi}{\partial x}(x, f(x))=0, & l_{0}<x<x_{0}, \\
|\nabla \varphi(x, \tilde{f}(x))|=c^{*}, & x>x_{0},
\end{array}
$$

where

$$
\tilde{f}(x)=f\left(x_{0}\right)+f^{\prime}\left(x_{0}\right)\left(x-x_{0}\right), \quad x \geq x_{0}
$$

and $\hat{\Omega}$ is the unbounded domain bounded by $\Gamma_{\text {in }}$, the $x$-axis, $y=f(x)\left(l_{0} \leq x \leq x_{0}\right)$ and $y=\tilde{f}(x)(x \geq$ $\left.x_{0}\right)$. Furthermore,

$$
\frac{\partial \varphi}{\partial x}(x, \tilde{f}(x))=\frac{c^{*}}{\sqrt{1+\left(f^{\prime}\left(x_{0}\right)\right)^{2}}}, \quad \frac{\partial \varphi}{\partial y}(x, \tilde{f}(x))=\frac{f^{\prime}\left(x_{0}\right) c^{*}}{\sqrt{1+\left(f^{\prime}\left(x_{0}\right)\right)^{2}}}, \quad x \geq x_{0}
$$

and

$$
\varphi(x, \tilde{f}(x))=\varphi\left(x_{0}, f\left(x_{0}\right)\right)+\frac{c^{*}}{\sqrt{1+\left(f^{\prime}\left(x_{0}\right)\right)^{2}}}\left(\left(x-x_{0}+f^{\prime}\left(x_{0}\right)\left(\tilde{f}(x)-f\left(x_{0}\right)\right)\right), \quad x \geq x_{0} .\right.
$$

Extend $\varphi$ from $\overline{\hat{\Omega}}$ to $\bar{\Omega}$ by defining

$$
\varphi(x, y)=\varphi\left(x_{0}, f\left(x_{0}\right)\right)+\frac{c^{*}}{\sqrt{1+\left(f^{\prime}\left(x_{0}\right)\right)^{2}}}\left(\left(x-x_{0}+f^{\prime}\left(x_{0}\right)\left(y-f\left(x_{0}\right)\right)\right), \quad(x, y) \in \bar{\Omega} \backslash \overline{\hat{\Omega}} .\right.
$$

Then, $\varphi \in C^{2}\left(\bar{\Omega} \backslash \bar{\Omega}_{\mathrm{v}}\right) \cap C^{1}(\bar{\Omega})$ is just a solution to the problem (2.31)-2.35) satisfying (i) according to Theorem 3.2 and Proposition 5.2. Moreover, (ii) follows from Theorem 4.1 .

To verify the uniqueness of the global smooth solution to the problem (2.31)-(2.35), it suffices to prove that if $\hat{\varphi} \in C^{1}\left(\bar{\Omega}_{\mathrm{v}}\right)$ solves

$$
\begin{array}{ll}
\operatorname{div}\left(\rho\left(|\nabla \hat{\varphi}|^{2}\right) \nabla \hat{\varphi}\right)=0, & (x, y) \in \Omega_{\mathrm{v}}, \\
\frac{\partial \hat{\varphi}}{\partial x}(x, \tilde{f}(x))=\frac{c^{*}}{\sqrt{1+\left(f^{\prime}\left(x_{0}\right)\right)^{2}}}, \quad \frac{\partial \hat{\varphi}}{\partial y}(x, \tilde{f}(x))=\frac{f^{\prime}\left(x_{0}\right) c^{*}}{\sqrt{1+\left(f^{\prime}\left(x_{0}\right)\right)^{2}}}, & x>x_{0},
\end{array}
$$




$$
\rho\left(|\nabla \hat{\varphi}(x, f(x))|^{2}\right)\left(\frac{\partial \hat{\varphi}}{\partial y}(x, f(x))-f^{\prime}(x) \frac{\partial \hat{\varphi}}{\partial x}(x, f(x))\right)=0, \quad x_{0}<x<l_{1},
$$

then $|\nabla \hat{\varphi}|=c^{*}$ in $\Omega_{\mathrm{v}}$. Note that the flow angle on $\partial \Omega_{\mathrm{v}} \cap \Omega$ equals $\arctan f^{\prime}\left(x_{0}\right) \in(0, \pi / 2)$. A similar argument as the beginning of the proof of Theorem 5.1 shows

$$
|\nabla \hat{\varphi}(x, y)|=c^{*}, \quad(x, y) \in \bar{\Omega}_{\mathrm{v}}, x-x_{0} \leq \delta
$$

with some positive constant $\delta$. Furthermore, one can prove that

$$
\frac{\partial \hat{\varphi}}{\partial x}(x, y)=\frac{c^{*}}{\sqrt{1+\left(f^{\prime}\left(x_{0}\right)\right)^{2}}}, \quad \frac{\partial \hat{\varphi}}{\partial y}(x, y)=\frac{f^{\prime}\left(x_{0}\right) c^{*}}{\sqrt{1+\left(f^{\prime}\left(x_{0}\right)\right)^{2}}}, \quad(x, y) \in \bar{\Omega}_{\mathrm{v}}, x-x_{0} \leq \delta .
$$

Repeating this discussion leads to

$$
|\nabla \hat{\varphi}(x, y)|=c^{*}, \quad \frac{\partial \hat{\varphi}}{\partial x}(x, y)=\frac{c^{*}}{\sqrt{1+\left(f^{\prime}\left(x_{0}\right)\right)^{2}}}, \quad \frac{\partial \hat{\varphi}}{\partial y}(x, y)=\frac{f^{\prime}\left(x_{0}\right) c^{*}}{\sqrt{1+\left(f^{\prime}\left(x_{0}\right)\right)^{2}}}, \quad(x, y) \in \bar{\Omega}_{\mathrm{v}} .
$$

We now prove (iii)-(v). Transform the supersonic flow on $\overline{\hat{\Omega}}$ into the potential plane. This transformation is one to one according to (5.28). It is assumed that $(\Upsilon(0), 0)$ and $\left(x_{0}, f\left(x_{0}\right)\right)$ are transformed into $(0,0)$ and $(\zeta, m)$, respectively, in the coordinates transformation from the physical plane to the potential plane. For $(\varphi, \psi) \in[0,+\infty) \times[0, m] \backslash[\zeta,+\infty) \times\{m\}$, set

$$
\begin{gathered}
W(\varphi, \psi)=Q_{\varphi}(\varphi, \psi)-b^{1 / 2}(Q(\varphi, \psi)) Q_{\psi}(\varphi, \psi), \quad Z(\varphi, \psi)=-Q_{\varphi}(\varphi, \psi)-b^{1 / 2}(Q(\varphi, \psi)) Q_{\psi}(\varphi, \psi), \\
\mathscr{U}(\varphi, \psi)=b^{1 / 2}\left(Q(\varphi, \psi) W(\varphi, \psi), \quad \mathscr{V}(\varphi, \psi)=b^{1 / 2}(Q(\varphi, \psi)) Z(\varphi, \psi) .\right.
\end{gathered}
$$

Then, $(W, Z, Q)$ satisfies

$$
W(\varphi, \psi) \leq 0, \quad Z(\varphi, \psi) \geq 0, \quad Q(\varphi, \psi) \leq-\mu_{1}, \quad(\varphi, \psi) \in(0,+\infty) \times(0, m),
$$

$(W, Z) \in C([0,+\infty) \times[0, m] \backslash[\zeta,+\infty) \times\{m\}) \times C([0,+\infty) \times[0, m] \backslash[\zeta,+\infty) \times\{m\})$ solving

$$
\begin{array}{ll}
W_{\varphi}+b^{1 / 2}(Q) W_{\psi}=\frac{1}{4} b^{-1}(Q) p(Q) W(W+Z), & (\varphi, \psi) \in(0,+\infty) \times(0, m), \\
Z_{\varphi}-b^{1 / 2}(Q) Z_{\psi}=-\frac{1}{4} b^{-1}(Q) p(Q) Z(W+Z), & (\varphi, \psi) \in(0,+\infty) \times(0, m),
\end{array}
$$

and $(\mathscr{U}, \mathscr{V}) \in C([0,+\infty) \times[0, m] \backslash[\zeta,+\infty) \times\{m\}) \times C([0,+\infty) \times[0, m] \backslash[\zeta,+\infty) \times\{m\})$ solving

$$
\begin{array}{ll}
\mathscr{U}_{\varphi}+b^{1 / 2}(Q) \mathscr{U}_{\psi}=\frac{1}{4} b^{-3 / 2}(Q) p(Q) \mathscr{U}(\mathscr{U}-\mathscr{V}) \geq 0, & (\varphi, \psi) \in(0,+\infty) \times(0, m), \\
\mathscr{V}_{\varphi}-b^{1 / 2}(Q) \mathscr{V}_{\psi}=\frac{1}{4} b^{-3 / 2}(Q) p(Q) \mathscr{V}(\mathscr{U}-\mathscr{V}) \leq 0, & (\varphi, \psi) \in(0,+\infty) \times(0, m), \\
\mathscr{U}(0, \psi)=\mathscr{U}_{0}(\psi)=b^{1 / 2}\left(Q_{0}(\psi)\right) G_{0}(\psi)-b\left(Q_{0}(\psi)\right) Q_{0}^{\prime}(\psi), & \psi \in(0, m), \\
\mathscr{V}(0, \psi)=\mathscr{V}_{0}(\psi)=-b^{1 / 2}\left(Q_{0}(\psi)\right) G_{0}(\psi)-b\left(Q_{0}(\psi)\right) Q_{0}^{\prime}(\psi), & \psi \in(0, m), \\
\mathscr{U}(\varphi, 0)+\mathscr{V}(\varphi, 0)=0, & \varphi \in(0,+\infty), \\
\mathscr{U}(\varphi, m)+\mathscr{V}(\varphi, m)=\frac{2 f^{\prime \prime}\left(X_{\text {up }}(\varphi)\right)\left(1+\left(f^{\prime}\left(X_{\text {up }}(\varphi)\right)\right)^{2}\right)^{-3 / 2}}{A_{+}^{-1}(Q(\varphi, m))}, & \varphi \in(0, \zeta), \\
\lim _{\psi \rightarrow m^{-}} Q(\varphi, \psi)=-\infty, & \varphi \in(\zeta,+\infty) .
\end{array}
$$


By the method of characteristics and Proposition 5.3, one can prove

$$
-M_{1} \leq \mathscr{U}(\varphi, \psi) \leq 0, \quad 0 \leq \mathscr{V}(\varphi, \psi) \leq M_{1}, \quad(\varphi, \psi) \in(0,+\infty) \times(0, m)
$$

with $M_{1}=M_{1}\left(\gamma, m, \mu_{1}, \mu_{3}, \zeta, f\right)$ being a positive constant. Therefore,

$$
-M_{1} \leq b^{1 / 2}(Q(\varphi, \psi)) Q_{\varphi}(\varphi, \psi) \leq 0, \quad\left|b(Q(\varphi, \psi)) Q_{\psi}(\varphi, \psi)\right| \leq M_{1}, \quad(\varphi, \psi) \in(0,+\infty) \times(0, m),
$$

which yields that $|\nabla \varphi|$ is globally Lipschitz continuous in $\Omega \backslash \bar{\Omega}_{\mathrm{v}}$. Thus (iii) is proved since $|\nabla \varphi|=c^{*}$ on $\bar{\Omega}_{\mathrm{v}}$.

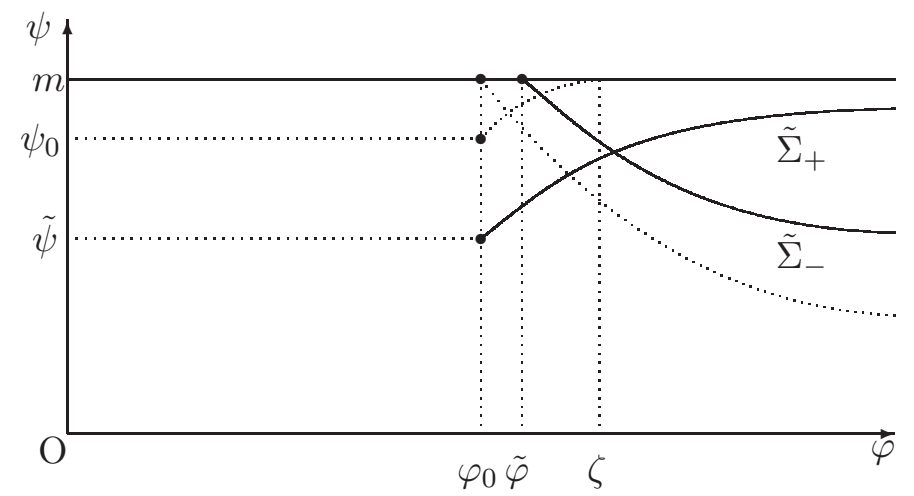

By Lemma 2.1 and Proposition [5.3, there exists $\varphi_{0} \in(0, \zeta)$ such that both the negative characteristic from $\left(\varphi_{0}, m\right)$ and the positive characteristic from $\left(\varphi_{0}, m / 2\right)$ are unbounded. Denote

$$
\psi_{0}=\sup \left\{\psi \in(0, m) \text { : the positive characteristic from }\left(\varphi_{0}, \psi\right) \text { is unbounded }\right\} .
$$

Fix $\tilde{\varphi} \in\left(\varphi_{0}, \zeta\right)$ and $\tilde{\psi} \in\left(0, \psi_{0}\right)$. Let $\tilde{\Sigma}_{+}$be the positive characteristic from $\left(\varphi_{0}, \tilde{\psi}\right)$ and $\tilde{\Sigma}_{-}$be the negative characteristic from $(\tilde{\varphi}, m)$, i.e.,

$$
\begin{array}{llll}
\tilde{\Sigma}_{+}: \tilde{\Psi}_{+}^{\prime}(\varphi)=b^{1 / 2}\left(Q\left(\varphi, \tilde{\Psi}_{+}(\varphi)\right)\right), & \tilde{\psi}<\tilde{\Psi}_{+}(\varphi)<m, \quad \varphi>\varphi_{0}, & \tilde{\Psi}_{+}\left(\varphi_{0}\right)=\tilde{\psi}, \\
\tilde{\Sigma}_{-}: \tilde{\Psi}_{-}^{\prime}(\varphi)=-b^{1 / 2}\left(Q\left(\varphi, \tilde{\Psi}_{-}(\varphi)\right)\right), & 0<\tilde{\Psi}_{-}(\varphi)<m, \quad \varphi>\tilde{\varphi}, & \tilde{\Psi}_{-}(\tilde{\varphi})=m .
\end{array}
$$

It follows from (5.29), (5.32) and (5.33) that

$$
\begin{aligned}
\frac{d}{d \varphi} \mathscr{U}\left(\varphi, \tilde{\Psi}_{+}(\varphi)\right) & \geq M_{2}\left(-Q\left(\varphi, \tilde{\Psi}_{+}(\varphi)\right)\right)^{(\gamma-1) / 2} \mathscr{U}^{2}\left(\varphi, \tilde{\Psi}_{+}(\varphi)\right), & \varphi>\varphi_{0}, \\
\frac{d}{d \varphi} \mathscr{V}\left(\varphi, \tilde{\Psi}_{-}(\varphi)\right) \leq-M_{2}\left(-Q\left(\varphi, \tilde{\Psi}_{-}(\varphi)\right)\right)^{(\gamma-1) / 2} \mathscr{V}^{2}\left(\varphi, \tilde{\Psi}_{-}(\varphi)\right), & & \varphi>\tilde{\varphi}
\end{aligned}
$$

with $M_{2}=M_{2}\left(\gamma, \mu_{1}\right)$ being a positive constant. Therefore,

$$
\begin{aligned}
& \mathscr{U}\left(\varphi, \tilde{\Psi}_{+}(\varphi)\right) \geq-\frac{1}{M_{2}}\left(\int_{\varphi_{0}}^{\varphi}\left(-Q\left(s, \tilde{\Psi}_{+}(s)\right)\right)^{(\gamma-1) / 2} d s\right)^{-1}, \quad \varphi>\varphi_{0}, \\
& \mathscr{V}\left(\varphi, \tilde{\Psi}_{-}(\varphi)\right) \leq \frac{1}{M_{2}}\left(\int_{\tilde{\varphi}}^{\varphi}\left(-Q\left(s, \tilde{\Psi}_{-}(s)\right)\right)^{(\gamma-1) / 2} d s\right)^{-1}, \quad \quad \varphi>\tilde{\varphi} .
\end{aligned}
$$

Letting $\tilde{\psi} \rightarrow \psi_{0}^{-}$in (5.34) and $\tilde{\varphi} \rightarrow \zeta^{-}$in (5.35), and using (i) and (5.29), one can get

$$
\lim _{\psi \rightarrow m^{-}} \min _{\zeta_{1} \leq \varphi \leq \zeta_{2}} \mathscr{U}(\varphi, \psi)=0, \quad \lim _{\psi \rightarrow m^{-}} \max _{\zeta_{1} \leq \varphi \leq \zeta_{2}} \mathscr{V}(\varphi, \psi)=0, \quad \zeta<\zeta_{1}<\zeta_{2} .
$$


Therefore,

$$
\lim _{\psi \rightarrow m^{-}} \min _{\zeta_{1} \leq \varphi \leq \zeta_{2}} b^{1 / 2}(Q(\varphi, \psi)) Q_{\varphi}(\varphi, \psi)=0, \quad \lim _{\psi \rightarrow m^{-}} \max _{\zeta_{1} \leq \varphi \leq \zeta_{2}}\left|b(Q(\varphi, \psi)) Q_{\psi}(\varphi, \psi)\right|=0, \quad \zeta<\zeta_{1}<\zeta_{2}
$$

which yield that for each $(\tilde{x}, \tilde{y}) \in \partial \Omega_{\mathrm{v}} \cap \Omega$,

$$
\lim _{\substack{(x, y) \rightarrow(\tilde{x}, \tilde{y}) \\(x, y) \in \Omega \backslash \Omega_{\mathrm{v}}}} \nabla|\nabla \varphi(x, y)|=(0,0), \quad \lim _{\substack{(x, y) \rightarrow(\tilde{x}, \tilde{y}) \\(x, y) \in \Omega \backslash \Omega_{\mathrm{v}}}}\left(c^{*}-|\nabla \varphi(x, y)|\right)^{1 / 2} \nabla \arctan \frac{\varphi_{y}(x, y)}{\varphi_{x}(x, y)}=(0,0) .
$$

Thus (iv) is proved since $|\nabla \varphi|=c^{*}$ on $\bar{\Omega}_{\mathrm{v}}$.

Finally, we prove (v). It follows from $f^{\prime \prime}\left(x_{0}\right)>0$ and (5.29) that

$$
\begin{aligned}
\frac{\lim _{\varphi \rightarrow \zeta^{-}} b^{1 / 2}(Q(\varphi, m)) Z(\varphi, m)}{} & \geq \lim _{\varphi \rightarrow \zeta^{-}} b^{1 / 2}(Q(\varphi, m))(W(\varphi, m)+Z(\varphi, m)) \\
& =\frac{2 f^{\prime \prime}\left(x_{0}\right)}{c^{*}\left(1+\left(f^{\prime}\left(x_{0}\right)\right)^{2}\right)^{3 / 2}}>0,
\end{aligned}
$$

which yields that $Z(\cdot, m)$ is positive in the left neighborhood of $\zeta$. Without loss of generality, it is assumed that $Z(\cdot, m)>0$ in $\left(\varphi_{0}, \zeta\right)$, where $\varphi_{0}$ is given above. Let $\tilde{\Sigma}_{+}$and $\tilde{\Sigma}_{-}$be the above characteristics. Denote

$$
\begin{array}{ccc}
\tilde{\mathscr{P}}_{+}(\varphi)=Q\left(\varphi, \tilde{\Psi}_{+}(\varphi)\right), \quad \tilde{\mathscr{W}}(\varphi)=W\left(\varphi, \tilde{\Psi}_{+}(\varphi)\right), \quad \varphi \geq \varphi_{0}, \\
\tilde{\mathscr{P}}_{-}(\varphi)=Q\left(\varphi, \tilde{\Psi}_{-}(\varphi)\right), \quad \tilde{\mathscr{Z}}(\varphi)=Z\left(\varphi, \tilde{\Psi}_{-}(\varphi)\right), \quad \varphi \geq \tilde{\varphi} .
\end{array}
$$

Then, (5.30) and (5.31) yield

$$
\left(h\left(\tilde{\mathscr{P}}_{+}(\varphi)\right) \tilde{\mathscr{W}}(\varphi)\right)^{\prime}=\frac{1}{4} b^{-1}\left(\tilde{\mathscr{P}}_{+}(\varphi)\right) p\left(\tilde{\mathscr{P}}_{+}(\varphi)\right) h\left(\tilde{\mathscr{P}}_{+}(\varphi)\right) \tilde{\mathscr{W}}^{2}(\varphi), \quad \varphi>\varphi_{0}
$$

and

$$
\begin{gathered}
\tilde{\mathscr{P}}_{-}^{\prime}(\varphi)=W\left(\varphi, \tilde{\Psi}_{-}(\varphi)\right), \quad \varphi>\tilde{\varphi}, \\
\left(h\left(\tilde{\mathscr{P}}_{-}(\varphi)\right) \tilde{\mathscr{Z}}(\varphi)\right)^{\prime}=-\frac{1}{4} b^{-1}\left(\tilde{\mathscr{P}}_{-}(\varphi)\right) p\left(\tilde{\mathscr{P}}_{-}(\varphi)\right) h\left(\tilde{\mathscr{P}}_{-}(\varphi)\right) \tilde{\mathscr{Z}}^{2}(\varphi), \quad \varphi>\tilde{\varphi}, \quad \tilde{\mathscr{Z}}(\tilde{\varphi})>0,
\end{gathered}
$$

where

$$
h(s)=\exp \left\{\frac{1}{4} \int_{-1}^{s} b^{-1}(t) p(t) d t\right\}, \quad s<0 .
$$

First estimate $\tilde{\mathscr{W}}$. It follows from (5.37) that

$$
h\left(\tilde{\mathscr{P}}_{+}(\varphi)\right) \tilde{\mathscr{W}}(\varphi) \geq h\left(\tilde{\mathscr{P}}_{+}\left(\varphi_{0}\right)\right) \tilde{\mathscr{W}}\left(\varphi_{0}\right), \quad \varphi>\varphi_{0}
$$

Note $\inf _{0<\tilde{\psi}<\psi_{0}} h\left(\tilde{\mathscr{P}}_{+}\left(\varphi_{0}\right)\right) \tilde{\mathscr{W}}\left(\varphi_{0}\right)>-\infty$. Then, (5.40) and (i) yield

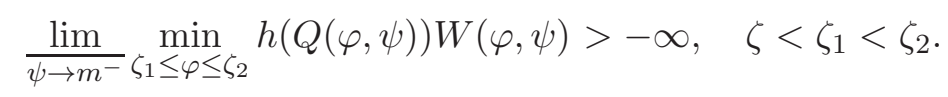

Turn to $\tilde{\mathscr{Z}}$. By (5.29), the second limit in (2.10) and $1<\gamma<3$, there exists a positive constant $M_{3}=M_{3}\left(\gamma, \mu_{1}\right)$ such that

$$
\frac{1}{4} b^{-1}(Q(\varphi, \psi)) p(Q(\varphi, \psi)) h^{-1}(Q(\varphi, \psi)) \leq M_{3}(-Q(\varphi, \psi))^{(\gamma-3) / 8}, \quad(\varphi, \psi) \in(0,+\infty) \times(0, m) .
$$


It follows from (5.29), (5.38), (5.39) and (5.42) that

$$
\begin{gathered}
\tilde{\mathscr{P}}_{-}(\varphi) \leq \tilde{\mathscr{P}}_{-}(\tilde{\varphi}), \quad \tilde{\mathscr{Z}}(\varphi)>0, \quad \varphi>\tilde{\varphi}, \\
\left(h\left(\tilde{\mathscr{P}}_{-}(\varphi)\right) \tilde{\mathscr{Z}}(\varphi)\right)^{\prime} \geq-M_{3}\left(-\tilde{\mathscr{P}}_{-}(\tilde{\varphi})\right)^{(\gamma-3) / 8}\left(h\left(\tilde{\mathscr{P}}_{-}(\varphi)\right) \tilde{\mathscr{Z}}(\varphi)\right)^{2}, \quad \varphi>\tilde{\varphi}
\end{gathered}
$$

and thus

$$
h\left(\tilde{\mathscr{P}}_{-}(\varphi)\right) \tilde{\mathscr{Z}}(\varphi) \geq \frac{1}{\left(h\left(\tilde{\mathscr{P}}_{-}(\tilde{\varphi})\right) \tilde{\mathscr{Z}}(\tilde{\varphi})\right)^{-1}+M_{3}\left(-\tilde{\mathscr{P}}_{-}(\tilde{\varphi})\right)^{(\gamma-3) / 8}(\varphi-\tilde{\varphi})}, \quad \varphi \geq \tilde{\varphi}
$$

Note that (15.36), the second limit in (2.10) and (i) give $\lim _{\tilde{\varphi} \rightarrow \zeta^{-}} h(\tilde{\mathscr{P}}-(\tilde{\varphi})) \tilde{\mathscr{Z}}(\tilde{\varphi})=+\infty$ and $\lim _{\tilde{\varphi} \rightarrow \zeta^{-}} \tilde{\mathscr{P}}_{-}(\tilde{\varphi})=$ $-\infty$. Therefore,

$$
\lim _{\psi \rightarrow m^{-}} \min _{\zeta_{1} \leq \varphi \leq \zeta_{2}} h(Q(\varphi, \psi)) Z(\varphi, \psi)=+\infty, \quad \zeta<\zeta_{1}<\zeta_{2} .
$$

Combine (5.41) and (5.43) to get

$$
\lim _{\tilde{\varphi} \rightarrow \zeta^{-}} \max _{\zeta_{1} \leq \varphi \leq \zeta_{2}} h(Q(\varphi, \psi)) b^{1 / 2}(Q(\varphi, \psi)) Q_{\psi}(\varphi, \psi)=-\infty, \quad \zeta<\zeta_{1}<\zeta_{2},
$$

which, together with the second limit in (2.10), yields

$$
\lim _{\tilde{\varphi} \rightarrow \zeta^{-}} \min _{\zeta_{1} \leq \varphi \leq \zeta_{2}}\left(c^{*}-q(\varphi, \psi)\right)^{(3-\gamma-\varepsilon) /(4 \gamma-4)} q_{\psi}(\varphi, \psi)=+\infty, \quad \zeta<\zeta_{1}<\zeta_{2}
$$

for each $\varepsilon>0$. Then, (v) is proved by transforming (5.44) into the physical plane.

\subsection{Smooth supersonic flows with vacuum at the inlet}

For an incoming flow with vacuum, the smooth supersonic flow problem can be formulated as follows

$$
\begin{array}{ll}
\operatorname{div}\left(\rho\left(|\nabla \varphi|^{2}\right) \nabla \varphi\right)=0, & (x, y) \in \Omega, \\
\rho\left(q_{0}^{2}(y)\right) \varphi(\Upsilon(y), y)=0, & 0<y<f\left(l_{0}\right), \\
|\nabla \varphi(\Upsilon(y), y)|=q_{0}(y), & 0<y<f\left(l_{0}\right) . \\
\rho\left(|\nabla \varphi(x, 0)|^{2}\right) \frac{\partial \varphi}{\partial y}(x, 0)=0, & \Upsilon(0)<x<l_{1}, \\
\rho\left(|\nabla \varphi(x, f(x))|^{2}\right)\left(\frac{\partial \varphi}{\partial y}(x, f(x))-f^{\prime}(x) \frac{\partial \varphi}{\partial x}(x, f(x))\right)=0, & l_{0}<x<l_{1} .
\end{array}
$$

Assume that $q_{0} \in C\left(\left[0, f\left(l_{0}\right)\right]\right) \cap C^{1}\left(\left[0, y_{1}\right]\right) \cap C^{1}\left(\left[y_{2}, f\left(l_{0}\right)\right]\right)$ with $0<y_{1}<y_{2}<f\left(l_{0}\right)$ satisfies

$$
q_{0}(y) \begin{cases}\in\left(c_{*}, c^{*}\right), & y \in\left[0, y_{1}\right) \cup\left(y_{2}, f\left(l_{0}\right)\right], \\ =c^{*}, & y \in\left[y_{1}, y_{2}\right]\end{cases}
$$

and

$$
\left|q_{0}^{\prime}(y)\right| \leq \frac{-\Upsilon^{\prime \prime}(y)}{1+\left(\Upsilon^{\prime}(y)\right)^{2}} \sqrt{\frac{-q_{0}^{2}(y) \rho\left(q_{0}^{2}(y)\right)}{\rho\left(q_{0}^{2}(y)\right)+2 q_{0}^{2}(y) \rho^{\prime}\left(q_{0}^{2}(y)\right)}}, \quad y \in\left[0, y_{1}\right) \cup\left(y_{2}, f\left(l_{0}\right)\right] .
$$

Similar to Proposition 5.2, one can solve the smooth supersonic flow problems with free boundary on $\left[0, y_{1}\right]$ and $\left[y_{2}, f\left(l_{0}\right)\right]$ individually. The two free boundaries are

$$
\tilde{\Gamma}_{k}: y=y_{k}-\Upsilon^{\prime}\left(y_{k}\right)\left(x-\Upsilon\left(y_{k}\right)\right), \quad x \geq \Upsilon\left(y_{k}\right),
$$


for $k=1,2$. Then, one can get a global smooth supersonic flow to the problem (5.45) -(5.49) by connecting the two smooth supersonic flows with free boundary by the vacuum.

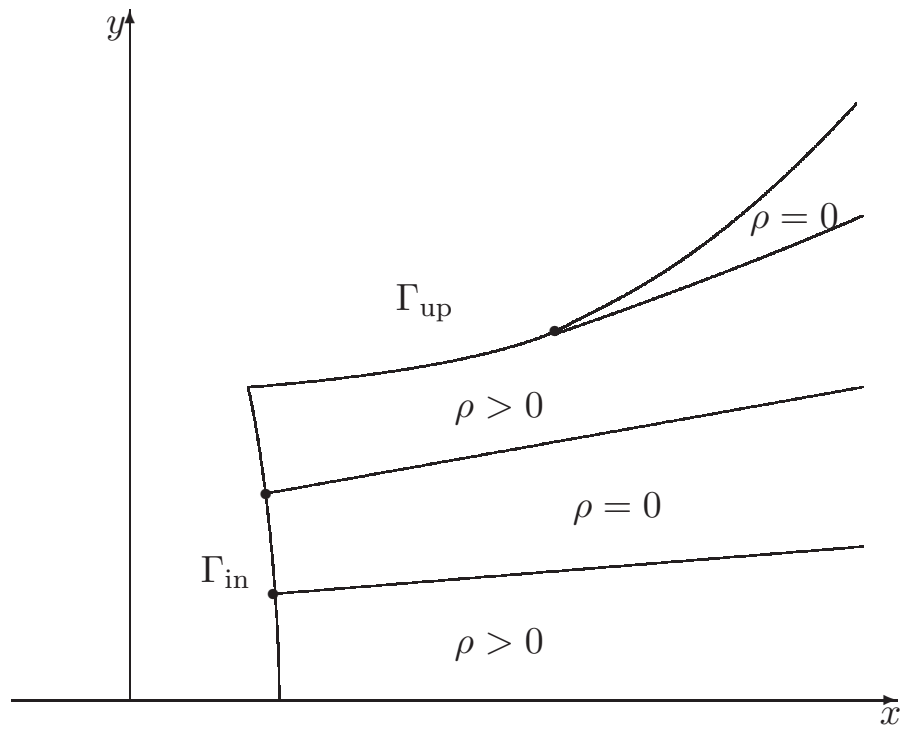

Theorem 5.3 Assume that $f \in C^{2}\left(\left[l_{0}, l_{1}\right)\right)$ satisfies (2.30), $\Upsilon \in C^{2}\left(\left[0, f\left(l_{0}\right)\right]\right)$ satisfies (2.36) and $q_{0} \in C\left(\left[0, f\left(l_{0}\right)\right]\right) \cap C^{1}\left(\left[0, y_{1}\right]\right) \cap C^{1}\left(\left[y_{2}, f\left(l_{0}\right)\right]\right)$ satisfies (5.50) and (5.51). Then the problem (5.45) (5.49) admits uniquely a global smooth solution $\varphi \in C^{1}(\bar{\Omega})$ with $|\nabla \varphi| \in C^{0,1}(\bar{\Omega})$. Besides the possible vacuum enclosure attached to the upper wall, there is another inner vacuum region which is the closed domain bounded by $\Gamma_{\mathrm{in}}, \tilde{\Gamma}_{1}$ and $\tilde{\Gamma}_{2}$. Moreover, $|\nabla \varphi| \in C^{1}(\Omega),\left.\frac{\partial|\nabla \varphi|}{\partial x}\right|_{\tilde{\Gamma}_{k} \cap \Omega}=\left.\frac{\partial|\nabla \varphi|}{\partial y}\right|_{\tilde{\Gamma}_{k} \cap \Omega}=0$ for $k=1,2$.

Remark 5.2 For the problem (5.45) -(5.49),

(i) If $0<y_{1}=y_{2}<f\left(l_{0}\right)$, then $\tilde{\Gamma}_{1}$ and $\tilde{\Gamma}_{2}$ coincide, and thus the inner vacuum region of the global smooth supersonic flow is degenerate to a half-line.

(ii) If $y_{1}=0$, then $\tilde{\Gamma}_{1}$ is the lower wall of the nozzle and the global smooth supersonic flow is vacuum on the lower wall.

(iii) If $y_{2}=f\left(l_{0}\right)$, then the vacuum enclosure attached to the upper wall and the inner vacuum region are connected, and thus the whole vacuum region is the closed domain bounded by $\Gamma_{\text {in }}, \Gamma_{\text {up }}$ and $\tilde{\Gamma}_{1}$.

\section{References}

[1] S. Canic, B. L. Keyfitz and G. M. Lieberman, A proof of existence of perturbed steady transonic shocks via a free boundary problem, Comm. Pure Appl. Math., 53(4)(2000), 484-511.

[2] G. Q. Chen and M. Feldman, Multidimensional transonic shocks and free boundary problems for nonlinear equations of mixed type, J. Amer. Math. Soc., 16(3)(2003), 461-494.

[3] G. Q. Chen and M. Feldman, Steady transonic shocks and free boundary problems for the Euler equations in infinite cylinders, Comm. Pure Appl. Math., 57(3)(2004), 310-356.

[4] G. Q. Chen and M. Feldman, Existence and stability of multidimensional transonic flows through an infinite nozzle of arbitrary cross-sections, Arch. Ration. Mech. Anal., 184(2)(2007), 185-242. 
[5] R. Courant and K. O. Friedrichs, Supersonic flow and shock waves, Interscience Publishers, Inc., New York, NY, 1948.

[6] J. Li, Z. P. Xin and H. C. Yin, On transonic shocks in a nozzle with variable end pressures, Comm. Math. Phys., 291(1)(2009), 111-150.

[7] J. Li, Z. P. Xin and H. C. Yin, A free boundary value problem for the full Euler system and 2-D transonic shock in a large variable nozzle, Math. Res. Lett., 16(5)(2009), 777-796.

[8] J. Li, Z. P. Xin and H. C. Yin, Transonic shocks for the full compressible Euler system in a general two-dimensional de Laval nozzle, Arch. Ration. Mech. Anal., 207(2)(2013), 533-581.

[9] T. P. Liu and T. Yang, Compressible Euler equations with vacuum, J. Differential Equations, 140(2)(1997), 223-237.

[10] C. P. Wang and Z. P. Xin, Smooth transonic flows in de Laval nozzles, arXiv preprint, arXiv:1304.2473, 2013.

[11] Z. P. Xin and H. C. Yin, Three-dimensional transonic shocks in a nozzle, Pacific J. Math., 236(1)(2008), 139-193.

[12] T. Yang, Singular behavior of vacuum states for compressible fluids, J. Comput. Appl. Math., 190(12)(2006), 211-231. 